\title{
Measurement of the production cross section of an isolated photon associated with jets in proton-proton collisions at $\sqrt{s}=7 \mathrm{TeV}$ with the ATLAS detector
}

\author{
G. Aad et al* \\ (ATLAS Collaboration) \\ (Received 14 March 2012; published 23 May 2012)
}

\begin{abstract}
A measurement of the cross section for the production of an isolated photon in association with jets in proton-proton collisions at a center-of-mass energy $\sqrt{s}=7 \mathrm{TeV}$ is presented. Photons are reconstructed in the pseudorapidity range $\left|\eta^{\gamma}\right|<1.37$ and with a transverse energy $E_{\mathrm{T}}^{\gamma}>25 \mathrm{GeV}$. Jets are reconstructed in the rapidity range $\left|y^{\text {jet }}\right|<4.4$ and with a transverse momentum $p_{\mathrm{T}}^{\text {jet }}>20 \mathrm{GeV}$. The differential cross section $d \sigma / d E_{\mathrm{T}}^{\gamma}$ is measured, as a function of the photon transverse energy, for three different rapidity ranges of the leading- $p_{\mathrm{T}}$ jet: $\left|y^{\text {jet }}\right|<1.2,1.2 \leq\left|y^{\text {jet }}\right|<2.8$ and $2.8 \leq\left|y^{\text {jet }}\right|<4$.4. For each rapidity configuration the same-sign $\left(\eta^{\gamma} y^{\text {jet }} \geq 0\right)$ and opposite-sign $\left(\eta^{\gamma} y^{\text {jet }}<0\right)$ cases are studied separately. The results are based on an integrated luminosity of $37 \mathrm{pb}^{-1}$, collected with the ATLAS detector at the LHC. Next-to-leading order perturbative QCD calculations are found to be in fair agreement with the data, except for $E_{\mathrm{T}}^{\gamma} \lesssim 45 \mathrm{GeV}$, where the theoretical predictions overestimate the measured cross sections.
\end{abstract}

DOI: 10.1103/PhysRevD.85.092014

PACS numbers: 13.85.Qk, 12.38.Qk

\section{INTRODUCTION}

At colliders, prompt photons are defined as photons produced in the beam particle collisions and not originating from particle decays. They include both direct photons, which originate from the hard process, and fragmentation photons, which arise from the fragmentation of a colored high- $p_{\mathrm{T}}$ parton $[1,2]$. At the LHC, the production of prompt photons in association with jets in proton-proton collisions, $p p \rightarrow \gamma+$ jet $+X$, represents an important test of perturbative QCD predictions at large hard-scattering scales $\left(Q^{2}\right)$ and over a wide range of the parton momentum fraction $(x)$. In addition the study of the angular correlations between the photon and the jet can be used to constrain the photon fragmentation functions [3]. Since the dominant $\gamma+$ jet production mechanism in $p p$ collisions at the LHC is through the $q g \rightarrow q \gamma$ process, the measurement of the photon + jet cross section at high rapidities and low transverse momenta can also be exploited to constrain the gluon density function inside the proton [3-6] for values of the incoming parton momentum fraction $x$ down to $\approx \mathcal{O}\left(10^{-3}\right)$. For the same reason, this final state can be used to obtain a high purity sample of quarkoriginated jets [7] that can be exploited to study detector performance with respect to these jets. The same events can also be used to calibrate the jet energy scale by profiting from momentum conservation in the transverse plane and the accurate energy measurement of the photon in the electromagnetic calorimeter [8]. Finally, $\gamma+$ jet events

*Full author list given at the end of the article.

Published by the American Physical Society under the terms of the Creative Commons Attribution 3.0 License. Further distribution of this work must maintain attribution to the author(s) and the published article's title, journal citation, and DOI. provide one of the main backgrounds in searches of Higgs bosons decaying to a photon pair [9]. An accurate knowledge of the photon + jet rate and angular distribution can be useful to understand the background level and shape in these searches.

In this article a measurement of the production cross section of an isolated prompt photon in association with jets, in $p p$ collisions at a center-of-mass energy $\sqrt{s}=$ $7 \mathrm{TeV}$, is presented. Photons are reconstructed in the pseudorapidity range of $\left|\eta^{\gamma}\right|<1.37$ and in the transverse energy range of $E_{\mathrm{T}}^{\gamma}>25 \mathrm{GeV}$. The same isolation criterion as used in our measurements of the inclusive isolated prompt photon $[10,11]$ and diphoton production cross sections [12] is used. It is based on the amount $E_{\mathrm{T}}^{\text {iso }}$ of transverse energy deposited in the calorimeters inside a cone of radius $R=\sqrt{\left(\eta-\eta^{\gamma}\right)^{2}+\left(\phi-\phi^{\gamma}\right)^{2}}=0.4$ centered around the photon direction (defined by $\eta^{\gamma}, \phi^{\gamma}$ ) [13]. The contribution from electromagnetic calorimeter cells in the $(\Delta \eta, \Delta \phi)=( \pm 0.0625, \pm 0.0875)$ region around the photon barycenter is not included in the sum. The mean value of the small leakage of the photon energy outside this region, evaluated as a function of the photon transverse energy, is subtracted from the measured value of $E_{\mathrm{T}}^{\text {iso }}$. The typical size of this correction is a few percent of the photon transverse energy. The measured value of $E_{\mathrm{T}}^{\text {iso }}$ is further corrected by subtracting the estimated contributions from the underlying event and additional inelastic $p p$ interactions. This correction is computed on an event-by-event basis using the method suggested in Refs. [14,15]. After the isolation requirement is applied, the relative contribution to the total cross section from fragmentation photons decreases, though it remains non-negligible especially at low transverse energies, below $35-40 \mathrm{GeV}$ [2]. The isolation requirement significantly reduces the main background, which consists of QCD multijet events where one 
jet typically contains a $\pi^{0}$ or $\eta$ meson which carries most of the jet energy and is misidentified as a prompt photon because it decays into a photon pair. Jets are reconstructed in the rapidity range of $\left|y^{\text {jet }}\right|<4.4$ and transverse momentum range of $p_{\mathrm{T}}^{\text {jet }}>20 \mathrm{GeV}$. The minimum separation between the highest $p_{\mathrm{T}}$ (leading) jet and the photon in the $\{\eta, \phi\}$ plane is $\Delta R>1.0$. The leading jet is required to be in either the central $\left(\left|y^{\text {jet }}\right|<1.2\right)$, forward $\left(1.2 \leq\left|y^{\text {jet }}\right|<2.8\right)$ or very forward $\left(2.8 \leq\left|y^{\text {jet }}\right|<4.4\right)$ rapidity interval.

The differential cross section $d \sigma / d E_{\mathrm{T}}^{\gamma}$ is measured for each of the three leading jet rapidity categories. Measurements are performed separately for the two cases where the photon pseudorapidity and the leading jet rapidity have same-sign $\left(\eta^{\gamma} y^{\text {jet }} \geq 0\right)$ or opposite-sign $\left(\eta^{\gamma} y^{\text {jet }}<0\right)$, and the results are compared to next-toleading order (NLO) perturbative QCD theoretical predictions. Separating the selected phase space into these six different angular configurations allows the comparison between data and theoretical predictions in configurations where the relative contribution of the fragmentation component to the total cross section is different, and in different ranges of $x$, which in the leading-order approximation is equal to $x=\frac{E_{\mathrm{T}}^{\gamma}}{\sqrt{s}}\left(e^{ \pm \eta^{\gamma}}+e^{ \pm y^{\text {jet }}}\right)$. The differential cross sections are measured up to $E_{\mathrm{T}}^{\gamma}=400 \mathrm{GeV}$ for the central and forward jet configurations, and up to $E_{\mathrm{T}}^{\gamma}=200 \mathrm{GeV}$ for the very forward jet configurations. These measurements cover the region $x \geqslant 0.001$ and $625 \mathrm{GeV}^{2} \leq Q^{2} \equiv$ $\left(E_{\mathrm{T}}^{\gamma}\right)^{2} \leq 1.6 \times 10^{5} \mathrm{GeV}^{2}$, thus extending the kinematic reach of previous photon + jet measurements at hadron [16-19] and electron-proton [20-23] colliders.

\section{THE ATLAS DETECTOR}

The ATLAS experiment [24] is a multipurpose particle physics detector with a forward-backward symmetric cylindrical geometry and nearly $4 \pi$ coverage in solid angle.

The inner tracking detector covers the pseudorapidity range $|\eta|<2.5$, and consists of a silicon pixel detector, a silicon microstrip detector, and, for $|\eta|<2.0$, a transition radiation tracker. The inner detector is surrounded by a thin superconducting solenoid providing a $2 \mathrm{~T}$ magnetic field.

The electromagnetic calorimeter is a lead-liquid argon sampling calorimeter. It is divided into a barrel section, covering the pseudorapidity region $|\eta|<1.475$, and two end-cap sections, covering the pseudorapidity regions $1.375<|\eta|<3.2$. It consists of three longitudinal layers in most of the pseudorapidity range. The first layer, with a thickness between 3 and 5 radiation lengths, is segmented into high granularity strips in the $\eta$ direction (width between 0.003 and 0.006 depending on $\eta$, with the exception of the regions $1.4<|\eta|<1.5$ and $|\eta|>2.4$ ), sufficient to provide event-by-event discrimination between singlephoton showers and two overlapping showers coming from a $\pi^{0}$ decay. The second layer of the electromagnetic calorimeter, which collects most of the energy deposited in the calorimeter by the photon shower, has a thickness around 17 radiation lengths and a cell granularity of $0.025 \times 0.025$ in $\eta \times \phi$. A third layer, with thickness varying between 4 and 15 radiation lengths, collects the tails of the electromagnetic showers and provides an additional point to reconstruct the shower barycenter. In front of the calorimeter a thin presampler layer, covering the pseudorapidity interval $|\eta|<1.8$, is used to correct for energy loss before the calorimeter. The electromagnetic energy scale is measured using $Z \rightarrow e e$ events with an uncertainty better than $1 \%$ [25]. The linearity has been found to be close to $1 \%$. At low $|\eta|$ the stochastic term is $(9-10) \% / \sqrt{E[\mathrm{GeV}]}$. However, it worsens as the amount of material in front of the calorimeter increases at larger $|\eta|$. The constant term is measured to be about $1.2 \%$ in the barrel and $1.8 \%$ in the end-cap region up to $|\eta|<2.47$ which is relevant for this analysis.

A hadronic sampling calorimeter is located outside the electromagnetic calorimeter. It is made of scintillating tiles and steel in the barrel section $(|\eta|<1.7)$, with depth around 7.4 interaction lengths, and of two end-caps of copper and liquid argon, with depth around 9 interaction lengths. Hadronic jets are reconstructed with an energy scale uncertainty of the order of $2.5 \%$ in the central to $14 \%$ in the very forward regions [26].

The muon spectrometer surrounds the calorimeters. It consists of three large air-core superconducting toroid systems, stations of precision tracking chambers providing accurate muon tracking over $|\eta|<2.7$, and detectors for triggering over $|\eta|<2.4$.

Events containing photon candidates are selected by a three-level trigger system. The first level trigger (level-1) is hardware based: using a trigger cell granularity $(0.1 \times 0.1$ in $\eta \times \phi$ ) coarser than that of the electromagnetic calorimeter, it searches for electromagnetic clusters within a fixed window of size $0.2 \times 0.2$ and retains only those whose total transverse energy in two adjacent trigger cells is above a programmable threshold. The algorithms of the second and third level triggers (collectively referred to as the high-level trigger) are implemented in software. The high-level trigger exploits the full granularity and precision of the calorimeter to refine the level-1 trigger selection, based on improved energy resolution and detailed information on energy deposition in the calorimeter cells.

\section{COLLISION DATA AND SIMULATED SAMPLES}

\section{A. Collision data}

The measurements presented here are based on $p p$ collision data collected at a center-of-mass energy $\sqrt{s}=$ $7 \mathrm{TeV}$ in 2010. Only events taken in stable beam conditions are considered and the trigger system, the tracking devices and the calorimeters are also required to be operational. Events are recorded using two single-photon 
triggers, with nominal transverse energy thresholds of 20 and $40 \mathrm{GeV}$. During the 2010 data-taking, no prescale was applied to the $40 \mathrm{GeV}$ threshold trigger and the corresponding total integrated luminosity of the collected sample amounts to $\int L d t=37.1 \mathrm{pb}^{-1}[27,28]$. In this measurement, this threshold is used to collect events in which the photon transverse energy, after reconstruction and calibration, is greater than $45 \mathrm{GeV}$. During the same data-taking period the average prescale of the $20 \mathrm{GeV}$ threshold trigger was 5.5, leading to a total integrated luminosity of $(6.7 \pm 0.2) \mathrm{pb}^{-1}$. This threshold is used in this measurement to collect events in which the photon transverse energy is lower than $45 \mathrm{GeV}$.

The selection criteria applied by the trigger on showershape variables computed from the energy profiles of the showers in the calorimeters are looser than the photon identification criteria applied in this measurement. Minimumbias events, triggered by two sets of scintillation counters located at $z= \pm 3.5 \mathrm{~m}$ from the collision center, are used to estimate the single-photon trigger efficiencies for true prompt photons with pseudorapidity $\left|\eta^{\gamma}\right|<2.37$. The efficiencies are constant and consistent with $100 \%$ within the uncertainty (Sec. VII) for $E_{\mathrm{T}}^{\gamma}>43 \mathrm{GeV}$ and $E_{\mathrm{T}}^{\gamma}>23 \mathrm{GeV}$ for the $40 \mathrm{GeV}$ and $20 \mathrm{GeV}$ threshold triggers, respectively.

In order to reduce noncollision backgrounds, events are required to have a reconstructed primary vertex with at least three associated tracks and consistent with the average beam spot position. The inefficiency of this requirement is negligible in true photon + jet events passing the acceptance criteria. The estimated contribution to the final photon sample from noncollision backgrounds is less than $0.1 \%$ and is therefore neglected $[10,11]$.

The total number of selected events in data after the trigger, data quality and primary vertex requirements is approximately six million.

\section{B. Simulated events}

To study the characteristics of signal and background events, simulated samples are generated using PYTHIA 6.423 [29]. The event generator parameters, including those of the underlying event model, are set according to the ATLAS AMBT1 tune [30], and the detector response is simulated using the GEANT4 program [31]. These samples are reconstructed with the same algorithms used for data. More details on the event generation and simulation infrastructure are provided in Ref. [32]. For the evaluation of systematic uncertainties related to the choice of the event generator and parton shower model, alternative samples are generated with HERWIG 6.510 [33]. The HERWIG event generation parameters are set according to the AUET1 tune [34] and the underlying event is generated using JIMMY 4.31 [35] with multiple parton interactions enabled.

The signal sample includes leading order $\gamma+$ jet events from both $q g \rightarrow q \gamma$ and $q \bar{q} \rightarrow g \gamma$ hard scattering and from quark bremsstrahlung in QCD dijet events. The background sample is generated by using all tree-level $2 \rightarrow 2$ QCD processes, removing $\gamma+$ jet events from quark bremsstrahlung.

The ratio between selected diphoton and inclusive photon + jet events is estimated to be $0.3 \%$ using PYTHIA diphoton samples. Therefore, background from diphoton events is neglected.

\section{PHOTON AND JET SELECTION}

\section{A. Photon selection}

Photons are reconstructed starting from clusters in the electromagnetic calorimeter with transverse energies exceeding $2.5 \mathrm{GeV}$, measured in projective towers of $3 \times 5$ cells in $\eta \times \phi$ in the second layer of the calorimeter. An attempt is made to match these clusters with tracks that are reconstructed in the inner detector and extrapolated to the calorimeter. Clusters without matching tracks are classified as unconverted photon candidates. Clusters with matched tracks are classified as electron candidates. To recover photon conversions, clusters matched to pairs of tracks originating from reconstructed conversion vertices in the inner detector or to single tracks with no hit in the innermost layer of the pixel detector are classified as converted photon candidates. The final energy measurement, for both converted and unconverted photons, is made using only the calorimeter, with a cluster size that depends on the photon classification. In the barrel, a cluster corresponding to $3 \times 5(\eta \times \phi)$ cells in the second layer is used for unconverted photons, while a cluster of $3 \times 7(\eta \times \phi)$ cells is used for converted photon candidates to compensate for the opening between the conversion products in the $\phi$ direction due to the magnetic field. In the end-cap, where the cell size along $\theta$ is smaller than in the barrel and the conversion tracks are closer in $\phi$ because of the smaller inner radius of the calorimeter, a cluster size of $5 \times 5$ is used for all candidates. A dedicated energy calibration [36] is then applied separately for converted and unconverted photon candidates to account for upstream energy loss and both lateral and longitudinal leakage. Both unconverted and converted photon candidates are considered for this measurement. Photons reconstructed near regions of the calorimeter affected by readout or high-voltage failures are not considered, eliminating around 5\% of the selected candidates. Events with at least one photon candidate with transverse energy $E_{\mathrm{T}}^{\gamma}>25 \mathrm{GeV}$ and pseudorapidity $\left|\eta^{\gamma}\right|<1.37$ are selected. Photons are selected using the same shower-shape and isolation variables discussed in Refs. [10,37]. The selection criteria on the shower-shape variables are independent of the photon candidate's transverse energy, but vary as a function of the photon reconstructed pseudorapidity, to take into account variations in the total thickness of the upstream material and in the calorimeter geometry. They are optimized independently for unconverted and converted photons to account for the 
different developments of the showers in each case. Applying these selection criteria suppresses backgrounds from jets misidentified as photons. The photon transverse isolation energy $E_{\mathrm{T}}^{\text {iso }}$ is required to be lower than $3 \mathrm{GeV}$. Less than $0.2 \%$ of events have more than one photon candidate passing the selection criteria. In such events the leading- $E_{\mathrm{T}}$ photon is retained.

\section{B. Jet selection}

Jets are reconstructed starting from three-dimensional topological clusters built from calorimeter cells, using the infrared- and collinear-safe anti- $k_{t}$ algorithm [38] with a radius parameter $R=0.4$. The jet four-momenta are constructed from a sum over their constituents, treating each as an $(E, \vec{p})$ four-vector with zero mass. The jet four-momenta are then recalibrated using a jet energy scale correction as described in Ref. [26]. The calibration procedure corrects for instrumental effects, such as inactive material and noncompensation, as well as for the additional energy due to multiple $p p$ interactions within the same bunch crossing (pile-up). Jets with calibrated transverse momenta greater than $20 \mathrm{GeV}$ are retained for this measurement.

To reject jets reconstructed from calorimeter signals not originating from a $p p$ collision, the same jet quality criteria used in Ref. [26] are applied here. These cuts suppress fake jets from calorimeter noise, cosmic rays and beamrelated backgrounds.

Jets overlapping with the candidate photon, or with an isolated electron produced from $W$ or $Z$ decay, are not considered. For this reason, if the jet axis is within a cone of radius 0.3 around the photon, the jet is discarded. Similarly, if the jet axis is within a cone of radius 0.3 around any electron that passes the tight identification criteria [25] and that has calorimeter isolation, $E_{\mathrm{T}}^{\text {iso }}$, less than $4 \mathrm{GeV}$, the jet is discarded.

The average jet multiplicity after the previous requirements is between 1.3 and 2.0, increasing with $E_{\mathrm{T}}^{\gamma}$. In events with multiple jet candidates, the leading- $p_{\mathrm{T}}$ jet is chosen. In order to retain the event, the leading jet is required to have rapidity $\left|y^{\text {jet }}\right|<4.4$. The leading jet axis is also required not to lie within a cone of radius $R=1.0$ around the photon direction.

The contamination in the selected sample from pile-up jets is estimated to be negligible, which is consistent with the low pile-up conditions of the 2010 data-taking, when, on average, only two minimum-bias events per bunch crossing are expected.

\section{Distribution of photon transverse energy in selected events}

The number of events after photon and jet selections is 213003. 96314 events have been collected with the $20 \mathrm{GeV}$ trigger and have $25 \mathrm{GeV}<E_{\mathrm{T}}^{\gamma} \leq 45 \mathrm{GeV}, 116689$ events have been collected with the $40 \mathrm{GeV}$ trigger and have $E_{\mathrm{T}}^{\gamma}>45 \mathrm{GeV}$. In $57 \%$ of the events the jet is central

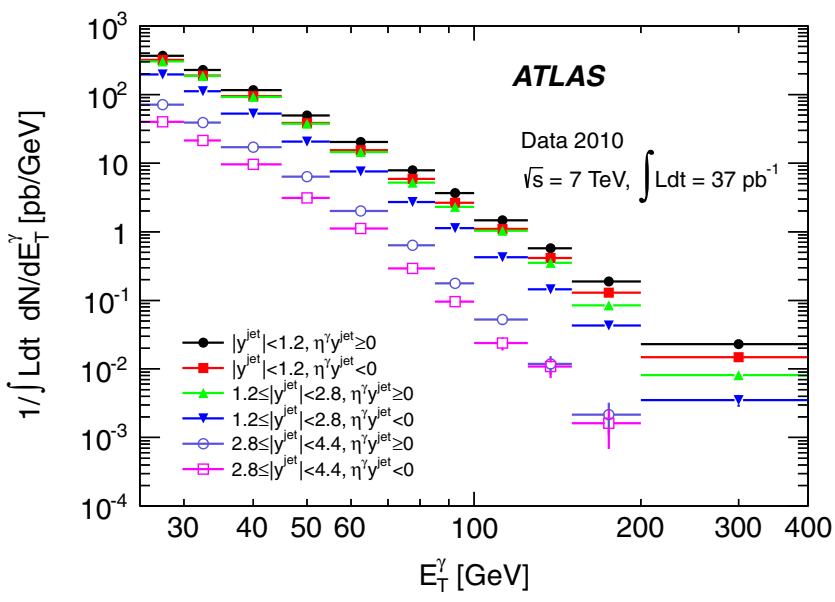

FIG. 1 (color online). Transverse energy distribution of photon candidates in photon + jet events selected in the 2010 ATLAS data, before background subtraction. The distribution is normalized by the integrated luminosity and the transverse energy bin width. Events with $E_{\mathrm{T}}^{\gamma} \leq 45 \mathrm{GeV}$ have been collected with the (prescaled) $20 \mathrm{GeV}$ photon trigger. Events with $E_{\mathrm{T}}^{\gamma}>45 \mathrm{GeV}$ have been collected with the (unprescaled) $40 \mathrm{GeV}$ photon trigger.

(32\%/25\% are in the same/opposite-sign configuration), in $37 \%$ of the events the jet is forward $(24 \% / 13 \%$ are in the same/opposite-sign configuration), and in $6 \%$ of the events the jet is very forward $(4 \% / 2 \%$ are in the same/oppositesign photon). The photon candidate is reconstructed as unconverted in $68 \%$ of the events and as converted in the remaining $32 \%$. The transverse energy distribution of the photon candidates in the selected sample is shown in Fig. 1.

\section{BACKGROUND SUBTRACTION AND SIGNAL YIELD ESTIMATION}

A non-negligible residual contribution of background is expected in the selected photon + jets sample, even after the application of the tight identification and isolation requirements. The dominant background is composed of dijet events in which one jet is misidentified as a prompt photon, with a tiny contribution from diphoton and $W / Z+$ jets events. In more than $95 \%$ of background dijet events, the misidentified jet contains a light neutral meson that carries most of the jet energy and decays to a collimated photon pair. The background yield in the selected sample is estimated in situ using a two-dimensional sideband technique as in Ref. [10] and then subtracted from the observed yield. In the background estimate, the photon is classified as:

(i) Isolated, if $E_{\mathrm{T}}^{\text {iso }}<3 \mathrm{GeV}$;

(ii) Nonisolated, if $E_{\mathrm{T}}^{\text {iso }}>5 \mathrm{GeV}$;

(iii) Tight, if it passes the tight photon identification criteria;

(iv) Nontight, if it fails at least one of the tight requirements on four shower-shape variables computed from the energy deposits in a few cells of the first 

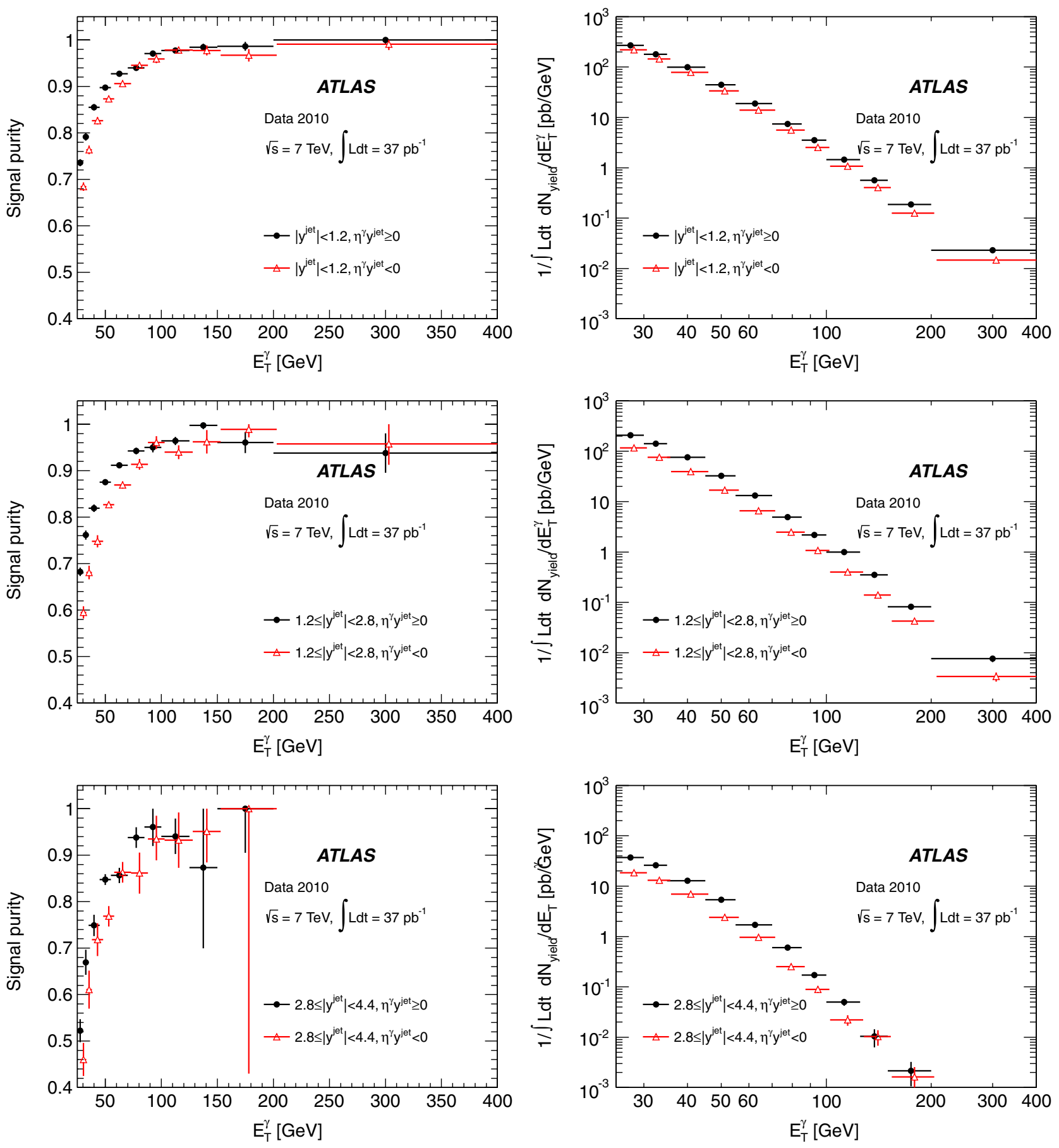

FIG. 2 (color online). Estimated signal purity (left column) and signal yield normalized by bin width and integrated luminosity (right column) in data as a function of the photon transverse energy, for the same-sign angular configurations (full circles) and the oppositesign angular configurations (open triangles). A small horizontal displacement has been added to the points corresponding to the opposite-sign configurations, so that the error bars are clearly shown. The errors are statistical only. Top row: central jet. Middle row: forward jet. Bottom row: very forward jet.

layer of the electromagnetic calorimeter, but passes all the other tight identification criteria.

In the two-dimensional plane [10] formed by the photon transverse isolation energy and the photon tight identification variable, we define four regions: (i) A: the signal region, containing tight, isolated photon candidates.

(ii) $B$ : the nonisolated background control region, containing tight, nonisolated photon candidates.

(iii) $C$ : the nonidentified background control region, containing isolated, nontight photon candidates. 
(iv) $D$ : the background control region containing nonisolated, nontight photon candidates.

The signal yield $N_{A}^{\text {sig }}$ in region $A$ is estimated from the number of events in the four regions, $N_{K}$ ( $K \in$ $\{A, B, C, D\})$, through the relation

$$
N_{A}^{\mathrm{sig}}=N_{A}-\left(N_{B}-c_{B} N_{A}^{\mathrm{sig}}\right) \frac{\left(N_{C}-c_{C} N_{A}^{\mathrm{sig}}\right)}{\left(N_{D}-c_{D} N_{A}^{\mathrm{sig}}\right)},
$$

where $c_{K} \equiv N_{K}^{\mathrm{sig}} / N_{A}^{\mathrm{sig}}$ are signal leakage fractions that can be extracted from simulated signal event samples. Equation (1) leads to a second-order polynomial equation in $N_{A}^{\text {sig }}$ that has only one physical $\left(N_{A}^{\text {sig }}>0\right)$ solution. The only hypothesis underlying Eq. (1) is that the isolation and identification variables are uncorrelated in background events. This assumption has been verified both in background simulated samples, and in data in the backgrounddominated region of $E_{\mathrm{T}}^{\text {iso }}>7 \mathrm{GeV}$. This method was found to return signal yields consistent with the generated ones using a cross section weighted combination of simulated signal and background samples.

The resulting signal purity and signal yield as a function of the photon candidate transverse energy for the six photon and jet angular configurations are shown in Fig. 2. The signal purity typically increases from between $50 \%$ and $70 \%$ at $E_{\mathrm{T}}^{\gamma}=25 \mathrm{GeV}$ to above $95 \%$ for $E_{\mathrm{T}}^{\gamma}>$ $150 \mathrm{GeV}$. The effect of the non-negligible signal leakage in the background control regions $\left(c_{K} \neq 0\right)$ increases the measured purity by $5-6 \%$ at $E_{\mathrm{T}}^{\gamma}=25 \mathrm{GeV}$ and $\approx 2 \%$ at $E_{\mathrm{T}}^{\gamma}>150 \mathrm{GeV}$ compared to the purity estimated assuming negligible signal in the background regions.

\section{SIGNAL EFFICIENCY AND CROSS SECTION MEASUREMENT}

The combined signal trigger, reconstruction, and selection efficiency is evaluated from the simulated signal samples described in Sec. IIIB, which include leading order $\gamma+$ jet events from both hard-scattering (hard subprocesses $q g \rightarrow q \gamma$ and $q \bar{q} \rightarrow g \gamma$ ) and from quark bremsstrahlung in QCD dijet events. For each of the six angular configurations, efficiency matrices $\left(\Lambda_{i j}\right)$ are constructed, with the indices $i$ and $j$ corresponding to reconstructed and true photon transverse energy intervals, respectively. The efficiency matrices account both for trigger, reconstruction, photon identification efficiencies and for migrations between different bins of the true and reconstructed photon transverse energies due to resolution effects. The matrix elements are determined from the ratios of two quantities. The denominators are defined in the following way:

(i) The leading truth-level signal photon within the acceptance $\left(\left|\eta_{\text {true }}^{\gamma}\right| \leq 1.37\right)$ is selected.

(ii) Truth jets are reconstructed using the anti- $k_{t}$ algorithm with a radius parameter $R=0.4$ on all the particles with proper lifetime longer than $10 \mathrm{ps}$, including photons, and the leading truth jet is selected among those with axis separated from the photon direction by $\Delta R>0.3$. The leading photon and the leading jet are required to be separated by $\Delta R>1.0$.

(iii) To retain the event the true leading photon is required to have $E_{\mathrm{T} \text {,true }}^{\gamma}>20 \mathrm{GeV}$ and to have a truth-particle-level isolation (computed from the true four-momenta of the generated particles inside a cone of radius 0.4 around the photon direction) $E_{\mathrm{T} \text {,true }}^{\text {iso }}<4 \mathrm{GeV}$. This truth-particle-level cut has been determined on PYTHIA photon + jet samples to match the efficiency of the experimental isolation cut at $3 \mathrm{GeV}$ (more details can be found in Ref. [10]). In this case, the same underlying event subtraction procedure used on data has been applied at the truth level. In addition, the leading truth jet is required to have $E_{\mathrm{T} \text {,true }}^{\mathrm{jet}}>20 \mathrm{GeV}$ and $\left|y_{\text {true }}^{\text {jet }}\right|<4$.4. At the truth level the minimum $E_{\mathrm{T} \text {,true }}^{\gamma}$ is set to $20 \mathrm{GeV}$ to account for possible migrations of photons with true transverse energy below $25 \mathrm{GeV}$ in the reconstructed transverse energy intervals above $25 \mathrm{GeV}$.

The numerators are determined by applying the selection criteria described in Sec. IV to the simulated signal samples. Since the simulation does not describe accurately the electromagnetic shower profiles, a correction factor for each simulated shape variable is applied to better match the data. We require the reconstructed isolation energy to be less than $3 \mathrm{GeV}$. As for the truth level, photons are allowed to have a $E_{\mathrm{T}, \text { reco }}^{\gamma}>20 \mathrm{GeV}$. The reconstructed photon is required to match the truth photon within a cone of radius 0.4 while the reconstructed jet is required to match the truth jet in a cone of radius 0.3 . Events which pass the selection at the reconstruction level but fail it at the truth level are properly accounted for in the normalization.

The event selection efficiency typically rises from $50 \%$ to $80 \%$ as a function of $E_{\mathrm{T}}^{\gamma}$. An inefficiency of around $15 \%$ is due to the acceptance loss originating from a few inoperative optical links in the calorimeter readout and from the isolation requirement. An inefficiency decreasing from $20-25 \%$ for $E_{\mathrm{T}}^{\gamma}=25 \mathrm{GeV}$ to almost zero at high $E_{\mathrm{T}}^{\gamma}$ originates from the shower-shape photon identification selection.

The differential cross section as a function of the photon true transverse energy $E_{\mathrm{T}}^{\text {true }}$ is computed in each bin $i$ of $E_{\mathrm{T}}^{\text {true }}$ and for each angular configuration $k$ as:

$$
\frac{d \sigma_{i}^{k}}{d E_{\mathrm{T}}^{\text {true }}}=\frac{N_{i}^{\gamma, \text { true, isol, }, k}}{\int L d t \Delta E_{\mathrm{T}, i}^{\text {true }}},
$$

where $N_{i}^{\gamma, \text { true, isol, } k}$ is the number of events containing a true isolated photon and hadronic jets, in which the true photon transverse energy is in bin $i$ and the angular configuration formed by the leading photon and jet is $k$. This number is 
related to the observed number of events passing the analysis cuts through the efficiency matrices $\Lambda_{i j}$ :

$$
N_{i}^{\gamma, \text { reco,isol }, k}=\sum_{j} \Lambda_{i j} N_{j}^{\gamma, \text { true }, \text { isol }, k}
$$

The unfolding procedure allows the reconstruction of the true number of events from the measured distribution, taking into account the measurement uncertainties due to statistical fluctuations in the finite measured sample. The simplest unfolding method is the basic bin-by-bin unfolding, which corrects the observed cross section in bin $i$ with the efficiency obtained from the ratio of selected events to truth events having the photon with reconstructed and true $E_{\mathrm{T}}$ in bin $i$. A more sophisticated method which properly accounts for migrations between bins is based on the repeated (iterative) application of Bayes's theorem [39]. The differences in the measured cross section for the two methods are a few percent for events with a central or forward jet and slightly higher for events with a very forward jet. Since the differences are within the statistical errors of the methods, we used the bin-by-bin method for these results.

\section{SYSTEMATIC UNCERTAINTIES}

We have considered the following sources of systematic uncertainties in the cross section measurement [40]:

(i) Simulation of the detector geometry. The presence of material in front of the calorimeter affects the photon conversion rate and the development of electromagnetic showers. Therefore the cross section measurement uncertainty depends on the accuracy of the detector simulation. The nominal simulation may underestimate the actual amount of material in front of the calorimeters. To quantify the effect of more material on the cross section, the full analysis is repeated using a detector simulation with a conservative estimate of additional material in front of the calorimeter [25]. In this case the photon identification and reconstruction efficiencies are lower than in the nominal case. The increase in cross section is assigned as a positive systematic uncertainty. In the central and forward jet configurations the systematic uncertainty varies from $5 \%$ to $8 \%$ for photons with $25 \mathrm{GeV}<E_{\mathrm{T}}^{\gamma} \leq 45 \mathrm{GeV}$ and from $1 \%$ to $5 \%$ for $E_{\mathrm{T}}^{\gamma}>45 \mathrm{GeV}$. In the very forward jet configurations the uncertainty is similarly estimated to range from $10 \%$ to $23 \%$.

(ii) Photon simulation. In order to take into account the uncertainty on the event generation and the parton shower model, four additional samples are used: PYTHIA or HERWIG samples containing only hardscattering photons and PYTHIA or HERWIG samples containing only photons from quark bremsstrahlung. The analysis is repeated using these samples, and the largest positive and negative deviations from the nominal cross section are taken as systematic uncertainties. The deviations are mainly positive, varying from $4 \%$ to $16 \%$ depending on $E_{\mathrm{T}}^{\gamma}$ or the angular configuration.

(iii) Jet and photon energy scale and resolution uncertainties. The cross section uncertainty is determined by varying the electromagnetic and the jet energy scales and resolutions within their uncertainties $[25,26]$. The effect on the cross section is found to be negligible, with the exception of the effect of the jet energy scale uncertainty, which affects mainly the first $E_{\mathrm{T}}^{\gamma}$ bin due to the efficiency of the $20 \mathrm{GeV}$ threshold on $p_{\mathrm{T}}^{\text {jet }}$. For the angular configurations including one central or one forward jet this effect is $3 \%$ to $7 \%$, for the configurations containing one very forward jet it is $9 \%$ to $20 \%$.

(iv) Uncertainty on the background correlation in the two-dimensional sidebands method. The isolation and identification variables are assumed to be independent for fake photon candidates. This assumption was verified using both data and simulated background samples and was found to be valid within a $10 \%$ uncertainty for configurations including a central or a forward jet and within a $25 \%$ uncertainty for configurations including a very forward jet. The cross section is recomputed accounting for these possible correlations in the background subtraction [10], and the difference with the nominal result is taken as a systematic uncertainty. This procedure gives a systematic uncertainty on the cross section of $3 \%$ and $6 \%$ in the first $E_{\mathrm{T}}^{\gamma}$ bin for these groups of configurations, respectively. This uncertainty decreases rapidly with increasing $E_{\mathrm{T}}^{\gamma}$, being proportional to $1-P$, where $P$ is the signal purity.

(v) Background control regions definition in the twodimensional sidebands method. The measurement is repeated using a different set of background identification or isolation criteria in the purity calculation, and the difference between the new cross section and the nominal result is taken as a systematic uncertainty. For background identification, three or five shower-shape variables are reversed instead of four as in the nominal case (more details can be found in Ref. [10]). The deviations on the cross section range from $5 \%$ in the central jet configurations to $12 \%$ in the forward jet configurations, all decreasing with increasing $E_{\mathrm{T}}^{\gamma}$. Varying the isolation cut by $\pm 1 \mathrm{GeV}$ results in less than $1 \%$ difference in the cross section.

(vi) Data-driven correction to the photon efficiency. The simulated photon shapes in the calorimeter have been corrected in order to improve the agreement with the data. The systematic uncertainty related to the correction procedure is computed 
using different simulated photon samples and a different simulation of the ATLAS detector and is estimated to be of the order of $1 \%$ to $4 \%$ in the first $E_{\mathrm{T}}^{\gamma}$ bin and lower than $1 \%$ elsewhere [11].

(vii) Uncertainty on the trigger efficiency. The trigger efficiency in the simulation is consistent with the one measured in data, using a bootstrap method, within the total uncertainty of the in situ measurement $\left(0.6 \%\right.$ uncertainty for $E_{\mathrm{T}}^{\gamma} \leq 45 \mathrm{GeV}$ and $0.4 \%$ for $\left.E_{\mathrm{T}}^{\gamma} \leq 45 \mathrm{GeV}\right)$. These uncertainties are added to the total systematic uncertainty on the cross section.

(viii) Uncertainty on the jet reconstruction efficiency. The simulation is found to reproduce data jet reconstruction efficiencies to better than $2 \%$ [41]. A $2 \%$ systematic uncertainty to the cross section is assigned.

(ix) Uncertainty on the simulated jet multiplicity. The LO generators used to estimate the signal efficiencies do not reproduce precisely the jet multiplicity observed in data, and the signal efficiency could depend on the multiplicity. Reweighting the simulation in order to reproduce the jet multiplicity observed in data changes the cross section by less than $1 \%$, which is taken as a systematic uncertainty.

(x) Uncertainty on the integrated luminosity. It has been determined to be $3.4 \%$ [27,28].

(xi) Isolated electron background. Possible backgrounds may arise from $W+$ jets where the $W$ decays into an electron misidentified as photon, and $W+\gamma$ where the $W$ decays into an electron misidentified as a jet. Additional backgrounds may originate from $Z \rightarrow e e$ where an electron may be misidentified as a photon, and combined with the jet arising from the misidentification of the other electron or with a jet from the rest of the event (in $Z+$ jets). Using simulated samples of these processes, scaled to their cross sections measured in [42-44], the total isolated electron background is estimated to be less than $1.5 \%$ of the signal yield measured in data in each photon $E_{\mathrm{T}}^{\gamma}$ bin. Therefore an asymmetric systematic uncertainty $\left({ }_{-1.5}^{+0.0}\right) \%$ on the measured cross section is assigned.

The sources of systematic uncertainty discussed above are considered as uncorrelated and thus the total systematic uncertainty (listed in the tables in Appendix B) is estimated by summing in quadrature all the contributions.

\section{THEORETICAL PREDICTIONS}

The expected production cross section of an isolated photon in association with jets as a function of the photon transverse energy $E_{\mathrm{T}}^{\gamma}$ is estimated using JETPHOX 1.3 [1]. JETPHOX is a parton-level Monte Carlo generator which implements a full NLO QCD calculation of both the direct and fragmentation contributions to the cross section. A parton-level isolation cut, requiring a total transverse energy below $4 \mathrm{GeV}$ from the partons produced with the photon inside a cone of radius $\Delta R=0.4$ in $\eta \times \phi$ around the photon direction, is used for this computation. The NLO photon fragmentation function [45] and the CT10 parton density functions [46] are used. The nominal renormalization $\left(\mu_{R}\right)$, factorization $\left(\mu_{F}\right)$ and fragmentation $\left(\mu_{f}\right)$ scales are set to the photon transverse energy $E_{\mathrm{T}}^{\gamma}$. Jets of partons are reconstructed by using an anti- $k_{T}$ algorithm with a radius parameter $R=0.4$. The same transverse momentum and rapidity criteria applied in the measurement to the reconstructed objects are used in the JETPHOX generation for the photon and the leading- $p_{\mathrm{T}}$ jet. As for data, the event is kept if the two objects are separated by $\Delta R>1.0$ in $\{\eta, \phi\}$. With this setup the fragmentation contribution to the total cross section decreases as a function of $E_{\mathrm{T}}^{\gamma}$, from $10 \%$ to $1.5 \%$ for the same-sign, central jet configuration while it varies from $22 \%$ to $2.5 \%$ in the same-sign, very forward jet configuration. In the opposite-sign configurations the fragmentation contribution is $20 \%$ to $50 \%$ (depending on $E_{\mathrm{T}}^{\gamma}$ and the jet rapidity) higher than in the corresponding same-sign configurations.

The JETPHOX cross section does not include underlying event, pile-up or hadronization effects. While the ambientenergy density correction of the photon isolation removes the effects from underlying event and pile-up on the photon side, potential differences between the JETPHOX theoretical cross section and the measured one may arise from the application of the jet selection, in particular, the transverse momentum threshold of $20 \mathrm{GeV}$. This cut is applied at parton-level in JETPHOX while it is applied to particle jets in the measured cross section and in the fully simulated PYTHIA and HERWIG samples.

One effect of hadronization is to spread energy outside of the jet area, so the jet $p_{\mathrm{T}}$ will tend to be lower than that of the originating parton(s); on the other hand, the underlying event adds extra particles to the jet candidate and results in the increase of the jet $p_{\mathrm{T}}$. To estimate these effects we use the simulated signal PYTHIA samples to evaluate the ratios of truth-level cross sections with and without hadronization and underlying event, and subsequently we multiply each bin of the JETPHOX cross sections by these ratios. These correction factors are smaller than 1 (around 0.9-0.95) at low $E_{\mathrm{T}}^{\gamma}$, indicating that the impact of hadronization on the jet $p_{\mathrm{T}}$ is more important than the extra energy added from the underlying event and pile-up. The correction factors are consistent with one at high $E_{\mathrm{T}}^{\gamma}$. This finding is in agreement with the expectations, since the photon and the jet transverse momenta are correlated and for large $E_{\mathrm{T}}^{\gamma}$ the $p_{\mathrm{T}}>20 \mathrm{GeV}$ cut becomes fully efficient both at parton- and particle-level.

The systematic uncertainties on the QCD cross sections computed with JETPHOX are estimated in the following way: 

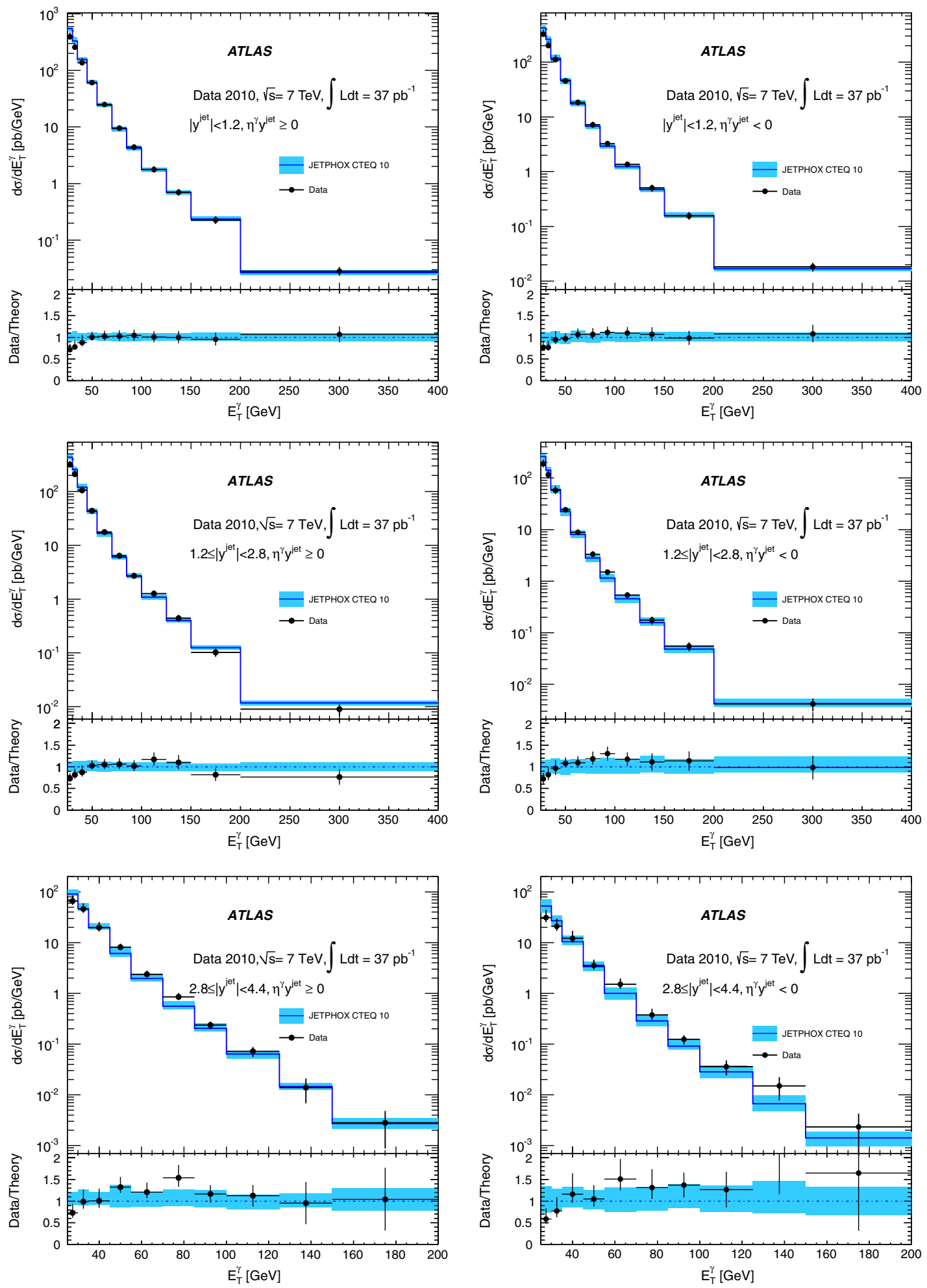

FIG. 3 (color online). Top graphs: experimental (black dots) and theoretical (blue line) photon + jet production cross sections, for the three same-sign (left column) and the three opposite-sign (right column) angular configurations. The black error bars represent the total experimental uncertainty. The blue bands show the total uncertainties on the theoretical predictions obtained with JETPHOX. Bottom graphs: ratio between the measured and the predicted cross sections. The blue bands show the theoretical uncertainties while the error bars show the experimental uncertainties on the ratio. First row: $\left|y^{\text {jet }}\right|<1.2$. Second row: $1.2 \leq\left|y^{\text {jet }}\right|<2.8$. Third row: $2.8 \leq\left|y^{\text {jet }}\right|<4.4$. 
(i) The scale uncertainty is evaluated by fixing any two scales to the nominal value and varying the third between 0.5 and 2.0 times the nominal value. In addition the effect of the coherent scale variations where all three scales are varied together is also taken into account. The envelope of the values obtained with the different scale configurations is taken as a systematic uncertainty. This leads to a change of the predicted cross section between $15 \%$ at low $E_{\mathrm{T}}^{\gamma}$ and $10 \%$ at high $E_{\mathrm{T}}^{\gamma}$.

(ii) The uncertainty on the cross section from the PDF uncertainty has been obtained by varying the PDFs within the $68 \%$ confidence level intervals. The corresponding uncertainty on the cross section varies between $5 \%$ and $2 \%$ as $E_{\mathrm{T}}^{\gamma}$ increases. Using a different set of PDFs, such as MSTW 2008 [47] or NNPDF 2.1 [48], the computed cross sections vary always within the total systematic uncertainty on the predicted cross section.

(iii) The uncertainty on the correspondence between parton-level and particle-level isolation cut has been evaluated by varying the cut between 3 and $5 \mathrm{GeV}$. This variation changes the predicted cross section by a few percent for the central configuration but becomes more important for the forward and very forward configurations, where the fragmentation contribution to the cross section is larger.

(iv) The uncertainty on the hadronization and underlying event corrections is estimated as the maximum spread of the correction factors obtained from PYTHIA using both the nominal and the Perugia 2010 tunes [49] and with HERWIG++ 2.5.1 with the UE7000-2 tune [50].

The expected cross sections with their full statistic and systematic uncertainties for all angular configurations under study are summarized in Appendix A.

\section{COMPARISON BETWEEN DATA AND THEORY}

The measured $E_{\mathrm{T}}^{\gamma}$-differential cross sections in the six photon-jet angular configurations under study are shown, with the theoretical cross sections overlaid, in Fig. 3. The ratio between data and theory is also plotted, showing the relative deviation of the measured cross section from the predicted cross section across the full $E_{\mathrm{T}}^{\gamma}$ range on a linear scale. The error bars represent the combination of statistical and systematic uncertainties, but are dominated by systematic uncertainties in all regions. The numerical results are presented in Appendix B.

The NLO pQCD predictions provided by JETPHOX are in fair agreement with the measured cross sections considering the given experimental and theoretical systematic uncertainties. As already observed in previous measurements of the inclusive prompt photon cross section at the LHC $[10,11,51]$, the data are consistently lower than the theoretical prediction in the $E_{\mathrm{T}}^{\gamma}<45 \mathrm{GeV}$ region, possibly suggesting an inaccuracy at low $E_{\mathrm{T}}^{\gamma}$ of the NLO predictions and the need to perform the theoretical calculations at a higher order in perturbation theory.

\section{CONCLUSION}

A measurement of the production cross section of an isolated prompt photon in association with jets in $p p$ collisions at a center-of-mass energy $\sqrt{s}=7 \mathrm{TeV}$ is presented. The measurement uses an integrated luminosity of $37 \mathrm{pb}^{-1}$ and covers the region $x \geq 0.001$ and $625 \mathrm{GeV}^{2} \leq$ $Q^{2} \leq 1.6 \times 10^{5} \mathrm{GeV}^{2}$, thus extending into kinematic regions previously unexplored with this final state at either hadron or electron-proton colliders. The differential cross section $d \sigma / d E_{\mathrm{T}}^{\gamma}$, as a function of the photon transverse energy, has been determined for isolated photons in the pseudorapidity range $\left|\eta^{\gamma}\right|<1.37$ and transverse energy $E_{\mathrm{T}}^{\gamma}>25 \mathrm{GeV}$, after integration over the jet transverse momenta for $p_{\mathrm{T}}^{\text {jet }}>20 \mathrm{GeV}$. A minimum separation of $\Delta R>1.0$ in the $\{\eta, \phi\}$ plane is required between the leading jet and the photon. The cross sections are presented separately for the three jet rapidity intervals $\left|y^{\text {jet }}\right|<1.2$, $1.2 \leq\left|y^{\text {jet }}\right|<2.8$ and $2.8 \leq\left|y^{\text {jet }}\right|<4.4$, distinguishing between the same-sign $\left(\eta^{\gamma} y^{\text {jet }} \geq 0\right)$ and opposite-sign $\left(\eta^{\gamma} y^{\text {jet }}<0\right)$ configurations. This subdivision allows the comparison between data and NLO perturbative QCD predictions in configurations where the relative contribution of the fragmentation component to the cross section and the explored ranges of the incoming parton momentum fraction $x$ are different. The NLO pQCD cross sections provided by JETPHOX are in fair agreement with the measured ones considering the typical (10\% to $30 \%)$ experimental and theoretical systematic uncertainties. In the $E_{\mathrm{T}}^{\gamma}<45 \mathrm{GeV}$ region, the NLO QCD calculation consistently overestimates the measured cross section, as observed in previous determinations of the inclusive prompt photon production cross section.

\section{ACKNOWLEDGMENTS}

We thank CERN for the very successful operation of the LHC, as well as the support staff from our institutions without whom ATLAS could not be operated efficiently. We acknowledge the support of ANPCyT, Argentina; YerPhI, Armenia; ARC, Australia; BMWF, Austria; ANAS, Azerbaijan; SSTC, Belarus; CNPq and FAPESP, Brazil; NSERC, NRC and CFI, Canada; CERN; CONICYT, Chile; CAS, MOST and NSFC, China; COLCIENCIAS, Colombia; MSMT CR, MPO CR and VSC CR, Czech Republic; DNRF, DNSRC and Lundbeck Foundation, Denmark; EPLANET and ERC, European Union; IN2P3-CNRS, CEA-DSM/IRFU, France; GNAS, Georgia; BMBF, DFG, HGF, MPG and AvH Foundation, Germany; GSRT, Greece; ISF, MINERVA, GIF, DIP and Benoziyo Center, Israel; INFN, Italy; MEXT and JSPS, Japan; CNRST, Morocco; FOM and NWO, Netherlands; RCN, Norway; MNiSW, Poland; 
GRICES and FCT, Portugal; MERYS (MECTS), Romania; MES of Russia and ROSATOM, Russian Federation; JINR; MSTD, Serbia; MSSR, Slovakia; ARRS and MVZT, Slovenia; DST/NRF, South Africa; MICINN, Spain; SRC and Wallenberg Foundation, Sweden; SER, SNSF and Cantons of Bern and Geneva, Switzerland; NSC, Taiwan; TAEK, Turkey; STFC, the Royal Society and Leverhulme Trust, United Kingdom; DOE and NSF, United States of America. The crucial computing support from all WLCG partners is acknowledged gratefully, in particular, from CERN and the ATLAS Tier-1 facilities at TRIUMF (Canada), NDGF (Denmark, Norway, Sweden),
CC-IN2P3 (France), KIT/GridKA (Germany), INFNCNAF (Italy), NL-T1 (Netherlands), PIC (Spain), ASGC (Taiwan), RAL (UK) and BNL (USA) and in the Tier-2 facilities worldwide.

\section{APPENDIX A: THEORETICAL photon + jet CROSS SECTION}

Tables I, II, III, IV, V, and VI show the theoretical photon + jet differential cross sections, in the six photonjet angular configurations under study, computed as described in Sec. VIII.

TABLE I. NLO pQCD cross section prediction for the production of an isolated photon in the pseudorapidity range $0.00 \leq$ $\left|\eta^{\gamma}\right|<1.37$ in association with a jet in the rapidity range $\left|y^{\text {jet }}\right|<1.2$ and $p_{\mathrm{T}}^{\text {jet }}>20 \mathrm{GeV}\left(\eta^{\gamma} y^{\text {jet }} \geq 0\right)$. The NLO pQCD cross section has been computed with JETPHOX 1.3 using CT10 PDFs. Details on the calculation of the uncertainties are discussed in Sec. VIII. In the last column the nonperturbative correction factor that must multiply the JETPHOX cross section is shown, with its uncertainty.

\begin{tabular}{|c|c|c|c|c|c|c|c|}
\hline $\begin{array}{l}E_{\mathrm{T}}^{\gamma} \min \\
{[\mathrm{GeV}]}\end{array}$ & $\begin{array}{c}E_{\mathrm{T}}^{\gamma} \max \\
{[\mathrm{GeV}]}\end{array}$ & $\begin{array}{c}\frac{d \sigma}{d E^{\gamma}} \\
{[\mathrm{pb} / \mathrm{GeV}]}\end{array}$ & $\begin{array}{l}\text { stat uncertainty } \\
{[\mathrm{pb} / \mathrm{GeV}]}\end{array}$ & $\begin{array}{l}\text { scale uncertainty } \\
{[\mathrm{pb} / \mathrm{GeV}]}\end{array}$ & $\begin{array}{l}\text { PDF uncertainty } \\
{[\mathrm{pb} / \mathrm{GeV}]}\end{array}$ & $\begin{array}{l}\text { isolation uncertainty } \\
{[\mathrm{pb} / \mathrm{GeV}]}\end{array}$ & $\begin{array}{l}\text { correction } \\
\text { factor }\end{array}$ \\
\hline 25 & 30 & 550 & \pm 6 & $\begin{array}{l}+41 \\
-41\end{array}$ & $\begin{array}{l}+28 \\
-36\end{array}$ & $\begin{array}{l}+9 \\
-0\end{array}$ & $0.927 \pm 0.036$ \\
\hline 30 & 35 & 331 & \pm 3 & $\begin{array}{l}+51 \\
-19\end{array}$ & $\begin{array}{l}+16 \\
-20\end{array}$ & $\begin{array}{l}+2 \\
-0\end{array}$ & $0.951 \pm 0.034$ \\
\hline 35 & 45 & 156 & \pm 1 & $\begin{array}{l}+14 \\
-13\end{array}$ & $\begin{array}{l}+7 \\
-8\end{array}$ & $\begin{array}{l}+0 \\
-2\end{array}$ & $0.983 \pm 0.026$ \\
\hline 45 & 55 & 60.4 & \pm 0.4 & $\begin{array}{l}+6.6 \\
-3.4\end{array}$ & $\begin{array}{l}+2.7 \\
-2.7\end{array}$ & $\begin{array}{l}+0.6 \\
-0.0\end{array}$ & $0.992 \pm 0.020$ \\
\hline 55 & 70 & 24.2 & \pm 0.2 & $\begin{array}{l}+2.1 \\
-1.6\end{array}$ & $\begin{array}{l}+1.0 \\
-0.9\end{array}$ & $\begin{array}{l}+0.0 \\
-0.3\end{array}$ & $1.002 \pm 0.025$ \\
\hline 70 & 85 & 9.26 & \pm 0.06 & $\begin{array}{l}+0.92 \\
-0.85\end{array}$ & $\begin{array}{l}+0.34 \\
-0.34\end{array}$ & $\begin{array}{l}+0.00 \\
-0.14\end{array}$ & $0.995 \pm 0.026$ \\
\hline 85 & 100 & 4.21 & \pm 0.03 & $\begin{array}{l}+0.37 \\
-0.34\end{array}$ & $\begin{array}{l}+0.14 \\
-0.14\end{array}$ & $\begin{array}{l}+0.01 \\
-0.04\end{array}$ & $1.001 \pm 0.022$ \\
\hline 100 & 125 & 1.76 & \pm 0.01 & $\begin{array}{l}+0.16 \\
-0.13\end{array}$ & $\begin{array}{l}+0.05 \\
-0.06\end{array}$ & $\begin{array}{l}+0.00 \\
-0.01\end{array}$ & $0.996 \pm 0.017$ \\
\hline 125 & 150 & 0.699 & \pm 0.004 & $\begin{array}{l}+0.061 \\
-0.056\end{array}$ & $\begin{array}{l}+0.019 \\
-0.020\end{array}$ & $\begin{array}{l}+0.005 \\
-0.007\end{array}$ & $0.992 \pm 0.018$ \\
\hline 150 & 200 & 0.236 & \pm 0.001 & $\begin{array}{l}+0.024 \\
-0.018\end{array}$ & $\begin{array}{l}+0.006 \\
-0.007\end{array}$ & $\begin{array}{l}+0.001 \\
-0.003\end{array}$ & $0.997 \pm 0.016$ \\
\hline 200 & 400 & 0.0266 & \pm 0.0001 & $\begin{array}{l}+0.0026 \\
-0.0023 \\
\end{array}$ & $\begin{array}{l}+0.0008 \\
-0.0008 \\
\end{array}$ & $\begin{array}{l}+0.0000 \\
-0.0002 \\
\end{array}$ & $0.988 \pm 0.026$ \\
\hline
\end{tabular}

TABLE II. NLO pQCD cross section prediction for the production of an isolated photon in the pseudorapidity range $0.00 \leq$ $\left|\eta^{\gamma}\right|<1.37$ in association with a jet in the rapidity range $\left|y^{\text {jet }}\right|<1.2$ and $p_{\mathrm{T}}^{\text {jet }}>20 \mathrm{GeV}\left(\eta^{\gamma} y^{\text {jet }}<0\right)$. The NLO pQCD cross section has been computed with JETPHOX 1.3 using CT10 PDFs. Details on the calculation of the uncertainties are discussed in Sec. VIII. In the last column the nonperturbative correction factor that must multiply the JETPHOX cross section is shown, with its uncertainty.

\begin{tabular}{|c|c|c|c|c|c|c|c|}
\hline $\begin{array}{l}E_{\mathrm{T}}^{\gamma} \min \\
{[\mathrm{GeV}]}\end{array}$ & $\begin{array}{c}E_{\mathrm{T}}^{\gamma} \max \\
{[\mathrm{GeV}]}\end{array}$ & $\begin{array}{c}\frac{d \sigma}{d E^{\gamma}} \\
{[\mathrm{pb} / \mathrm{GeV}]}\end{array}$ & $\begin{array}{l}\text { stat uncertainty } \\
{[\mathrm{pb} / \mathrm{GeV}]}\end{array}$ & $\begin{array}{l}\text { scale uncertainty } \\
{[\mathrm{pb} / \mathrm{GeV}]}\end{array}$ & $\begin{array}{l}\text { PDF uncertainty } \\
{[\mathrm{pb} / \mathrm{GeV}]}\end{array}$ & $\begin{array}{c}\text { isolation uncertainty } \\
{[\mathrm{pb} / \mathrm{GeV}]}\end{array}$ & $\begin{array}{l}\text { correction } \\
\text { factor }\end{array}$ \\
\hline 25 & 30 & 420 & \pm 5 & $\begin{array}{l}+49 \\
-40\end{array}$ & $\begin{array}{l}+21 \\
-29\end{array}$ & $\begin{array}{l}+8 \\
+0\end{array}$ & $0.925 \pm 0.041$ \\
\hline 30 & 35 & 261 & \pm 2 & $\begin{array}{l}+27 \\
-40\end{array}$ & $\begin{array}{l}+11 \\
-21\end{array}$ & $\begin{array}{l}+0 \\
-7\end{array}$ & $0.943 \pm 0.049$ \\
\hline 35 & 45 & 118 & \pm 1 & $\begin{array}{l}+17 \\
-16\end{array}$ & $\begin{array}{l}+6 \\
-6\end{array}$ & $\begin{array}{l}+2 \\
-0\end{array}$ & $0.980 \pm 0.032$ \\
\hline 45 & 55 & 47.0 & \pm 0.3 & $\begin{array}{l}+4.3 \\
-6.6\end{array}$ & $\begin{array}{l}+2.6 \\
-1.9\end{array}$ & $\begin{array}{l}+0.0 \\
-0.7\end{array}$ & $0.979 \pm 0.029$ \\
\hline 55 & 70 & 17.2 & \pm 0.1 & $\begin{array}{l}+2.8 \\
-1.6\end{array}$ & $\begin{array}{l}+0.8 \\
-0.7\end{array}$ & $\begin{array}{l}+0.0 \\
-0.5\end{array}$ & $0.982 \pm 0.025$ \\
\hline 70 & 85 & 6.72 & \pm 0.05 & $\begin{array}{l}+0.62 \\
-0.74\end{array}$ & $\begin{array}{l}+0.28 \\
-0.26\end{array}$ & $\begin{array}{l}+0.03 \\
-0.02\end{array}$ & $0.995 \pm 0.018$ \\
\hline 85 & 100 & 2.93 & \pm 0.02 & $\begin{array}{l}+0.34 \\
-0.25\end{array}$ & $\begin{array}{l}+0.11 \\
-0.11\end{array}$ & $\begin{array}{l}+0.03 \\
-0.00\end{array}$ & $0.981 \pm 0.031$ \\
\hline 100 & 125 & 1.24 & \pm 0.01 & $\begin{array}{l}+0.14 \\
-0.12\end{array}$ & $\begin{array}{l}+0.04 \\
-0.04\end{array}$ & $\begin{array}{l}+0.01 \\
-0.02\end{array}$ & $0.989 \pm 0.025$ \\
\hline 125 & 150 & 0.469 & \pm 0.003 & $\begin{array}{l}+0.053 \\
-0.039\end{array}$ & $\begin{array}{l}+0.015 \\
-0.016\end{array}$ & $\begin{array}{l}+0.005 \\
-0.007\end{array}$ & $0.992 \pm 0.027$ \\
\hline 150 & 200 & 0.159 & \pm 0.001 & $\begin{array}{l}+0.020 \\
-0.015\end{array}$ & $\begin{array}{l}+0.005 \\
-0.005\end{array}$ & $\begin{array}{l}+0.001 \\
-0.001\end{array}$ & $0.984 \pm 0.019$ \\
\hline 200 & 400 & 0.0169 & \pm 0.0001 & $\begin{array}{l}+0.0017 \\
-0.0015 \\
\end{array}$ & $\begin{array}{l}+0.0007 \\
-0.0007 \\
\end{array}$ & $\begin{array}{l}+0.0003 \\
-0.0000 \\
\end{array}$ & $0.991 \pm 0.026$ \\
\hline
\end{tabular}


TABLE III. NLO pQCD cross section prediction for the production of an isolated photon in the pseudorapidity range $0.00 \leq$ $\left|\eta^{\gamma}\right|<1.37$ in association with a jet in the rapidity range $1.2 \leq\left|y^{\text {jet }}\right|<2.8$ and $p_{\mathrm{T}}^{\text {jet }}>20 \mathrm{GeV}\left(\eta^{\gamma} y^{\text {jet }} \geq 0\right)$. The NLO pQCD cross section has been computed with JETPHOX 1.3 using CT10 PDFs. Details on the calculation of the uncertainties are discussed in Sec. VIII. In the last column the nonperturbative correction factor that must multiply the JETPHOX cross section is shown, with its uncertainty.

\begin{tabular}{|c|c|c|c|c|c|c|c|}
\hline $\begin{array}{l}E_{\mathrm{T}}^{\gamma} \min \\
{[\mathrm{GeV}]}\end{array}$ & $\begin{array}{c}E_{\mathrm{T}}^{\gamma} \max \\
{[\mathrm{GeV}]}\end{array}$ & $\begin{array}{c}\frac{d \sigma}{d E^{\gamma}} \\
{[\mathrm{pb} / \mathrm{GeV}]}\end{array}$ & $\begin{array}{l}\text { stat uncertainty } \\
{[\mathrm{pb} / \mathrm{GeV}]}\end{array}$ & $\begin{array}{l}\text { scale uncertainty } \\
{[\mathrm{pb} / \mathrm{GeV}]}\end{array}$ & $\begin{array}{l}\text { PDF uncertainty } \\
{[\mathrm{pb} / \mathrm{GeV}]}\end{array}$ & $\begin{array}{l}\text { isolation uncertainty } \\
{[\mathrm{pb} / \mathrm{GeV}]}\end{array}$ & $\begin{array}{l}\text { correction } \\
\text { factor }\end{array}$ \\
\hline 25 & 30 & 434 & \pm 5 & $\begin{array}{l}+49 \\
-35\end{array}$ & $\begin{array}{l}+18 \\
-23\end{array}$ & $\begin{array}{c}+3 \\
-10\end{array}$ & $0.925 \pm 0.054$ \\
\hline 30 & 35 & 258 & \pm 2 & $\begin{array}{l}+34 \\
-30\end{array}$ & $\begin{array}{c}+9 \\
-12\end{array}$ & $\begin{array}{l}+0 \\
-5\end{array}$ & $0.955 \pm 0.034$ \\
\hline 35 & 45 & 120 & \pm 1 & $\begin{array}{l}+17 \\
-14\end{array}$ & $\begin{array}{l}+4 \\
-5\end{array}$ & $\begin{array}{l}+0 \\
-4\end{array}$ & $0.988 \pm 0.021$ \\
\hline 45 & 55 & 42.4 & \pm 0.3 & $\begin{array}{l}+6.5 \\
-3.7\end{array}$ & $\begin{array}{l}+1.1 \\
-1.5\end{array}$ & $\begin{array}{l}+0.9 \\
-0.0\end{array}$ & $0.993 \pm 0.023$ \\
\hline 55 & 70 & 16.7 & \pm 0.1 & $\begin{array}{l}+1.8 \\
-2.0\end{array}$ & $\begin{array}{l}+0.4 \\
-0.5\end{array}$ & $\begin{array}{l}+0.5 \\
-0.0\end{array}$ & $0.997 \pm 0.025$ \\
\hline 70 & 85 & 6.02 & \pm 0.05 & $\begin{array}{l}+0.68 \\
-0.55\end{array}$ & $\begin{array}{l}+0.12 \\
-0.17\end{array}$ & $\begin{array}{l}+0.05 \\
-0.00\end{array}$ & $0.994 \pm 0.015$ \\
\hline 85 & 100 & 2.66 & \pm 0.02 & $\begin{array}{l}+0.30 \\
-0.24\end{array}$ & $\begin{array}{l}+0.05 \\
-0.07\end{array}$ & $\begin{array}{l}+0.02 \\
-0.01\end{array}$ & $0.989 \pm 0.020$ \\
\hline 100 & 125 & 1.09 & \pm 0.01 & $\begin{array}{l}+0.11 \\
-0.10\end{array}$ & $\begin{array}{l}+0.02 \\
-0.03\end{array}$ & $\begin{array}{l}+0.01 \\
-0.01\end{array}$ & $0.997 \pm 0.020$ \\
\hline 125 & 150 & 0.401 & \pm 0.003 & $\begin{array}{l}+0.051 \\
-0.035\end{array}$ & $\begin{array}{l}+0.007 \\
-0.009\end{array}$ & $\begin{array}{l}+0.002 \\
-0.004\end{array}$ & $0.998 \pm 0.020$ \\
\hline 150 & 200 & 0.125 & \pm 0.001 & $\begin{array}{l}+0.010 \\
-0.011\end{array}$ & $\begin{array}{l}+0.003 \\
-0.003\end{array}$ & $\begin{array}{l}+0.000 \\
-0.002\end{array}$ & $0.994 \pm 0.023$ \\
\hline 200 & 400 & 0.0118 & \pm 0.0001 & $\begin{array}{l}+0.0011 \\
-0.0012 \\
\end{array}$ & $\begin{array}{l}+0.0003 \\
-0.0003 \\
\end{array}$ & $\begin{array}{l}+0.0000 \\
-0.0001 \\
\end{array}$ & $0.993 \pm 0.017$ \\
\hline
\end{tabular}

TABLE IV. NLO pQCD cross section prediction for the production of an isolated photon in the pseudorapidity range $0.00 \leq$ $\left|\eta^{\gamma}\right|<1.37$ in association with a jet in the rapidity range $1.2 \leq\left|y^{\text {jet }}\right|<2.8$ and $p_{\mathrm{T}}^{\text {jet }}>20 \mathrm{GeV}\left(\eta^{\gamma} y^{\text {jet }}<0\right)$. The NLO pQCD cross section has been computed with JETPHOX 1.3 using CT10 PDFs. Details on the calculation of the uncertainties are discussed in Sec. VIII. In the last column the nonperturbative correction factor that must multiply the JETPHOX cross section is shown, with its uncertainty.

\begin{tabular}{|c|c|c|c|c|c|c|c|}
\hline $\begin{array}{l}E_{\mathrm{T}}^{\gamma} \min \\
{[\mathrm{GeV}]}\end{array}$ & $\begin{array}{c}E_{\mathrm{T}}^{\gamma} \max \\
{[\mathrm{GeV}]}\end{array}$ & $\begin{array}{c}\frac{d \sigma}{d E^{\gamma}} \\
{[\mathrm{pb} / \mathrm{GeV}]}\end{array}$ & $\begin{array}{l}\text { stat uncertainty } \\
{[\mathrm{pb} / \mathrm{GeV}]}\end{array}$ & $\begin{array}{l}\text { scale uncertainty } \\
{[\mathrm{pb} / \mathrm{GeV}]}\end{array}$ & $\begin{array}{l}\text { PDF uncertainty } \\
{[\mathrm{pb} / \mathrm{GeV}]}\end{array}$ & $\begin{array}{c}\text { isolation } \\
\text { uncertainty }[\mathrm{pb} / \mathrm{GeV}]\end{array}$ & $\begin{array}{l}\text { correction } \\
\text { factor }\end{array}$ \\
\hline 25 & 30 & 260 & \pm 3 & $\begin{array}{l}+33 \\
-44\end{array}$ & $\begin{array}{l}+13 \\
-12\end{array}$ & $\begin{array}{c}+0 \\
-17\end{array}$ & $0.935 \pm 0.075$ \\
\hline 30 & 35 & 141 & \pm 1 & $\begin{array}{l}-44 \\
-23 \\
-23\end{array}$ & $\begin{array}{l}-12 \\
+7\end{array}$ & $\begin{array}{l}-11 \\
+0 \\
-4\end{array}$ & $0.909 \pm 0.055$ \\
\hline 35 & 45 & 60 & \pm 1 & $\begin{array}{l}+12 \\
-9\end{array}$ & $\begin{array}{l}+3 \\
-2\end{array}$ & $\begin{array}{l}+1 \\
-0\end{array}$ & $0.975 \pm 0.034$ \\
\hline 45 & 55 & 22.3 & \pm 0.2 & $\begin{array}{l}+3.8 \\
-4.0\end{array}$ & $\begin{array}{l}+0.8 \\
-0.8\end{array}$ & $\begin{array}{l}+0.0 \\
-1.0\end{array}$ & $0.962 \pm 0.051$ \\
\hline 55 & 70 & 8.1 & \pm 0.1 & $\begin{array}{l}+1.5 \\
-1.1\end{array}$ & $\begin{array}{l}+0.3 \\
-0.3\end{array}$ & $\begin{array}{l}+0.0 \\
-0.3\end{array}$ & $0.961 \pm 0.047$ \\
\hline 70 & 85 & 2.81 & \pm 0.02 & $\begin{array}{l}+0.40 \\
-0.45\end{array}$ & $\begin{array}{l}+0.09 \\
-0.09\end{array}$ & $\begin{array}{l}+0.06 \\
-0.07\end{array}$ & $0.985 \pm 0.024$ \\
\hline 85 & 100 & 1.14 & \pm 0.01 & $\begin{array}{l}+0.24 \\
-0.18\end{array}$ & $\begin{array}{l}+0.04 \\
-0.04\end{array}$ & $\begin{array}{l}+0.00 \\
-0.02\end{array}$ & $0.998 \pm 0.035$ \\
\hline 100 & 125 & 0.456 & \pm 0.004 & $\begin{array}{l}+0.078 \\
-0.069\end{array}$ & $\begin{array}{l}+0.016 \\
-0.016\end{array}$ & $\begin{array}{l}+0.002 \\
-0.012\end{array}$ & $0.974 \pm 0.036$ \\
\hline 125 & 150 & 0.157 & \pm 0.002 & $\begin{array}{l}+0.040 \\
-0.019\end{array}$ & $\begin{array}{l}+0.006 \\
-0.006\end{array}$ & $\begin{array}{l}+0.002 \\
-0.000\end{array}$ & $0.979 \pm 0.040$ \\
\hline 150 & 200 & 0.0481 & \pm 0.0005 & $\begin{array}{l}+0.0086 \\
-0.0076\end{array}$ & $\begin{array}{l}+0.0022 \\
-0.0022\end{array}$ & $\begin{array}{l}+0.0010 \\
-0.0000\end{array}$ & $0.979 \pm 0.031$ \\
\hline 200 & 400 & 0.00422 & \pm 0.00005 & $\begin{array}{l}+0.00099 \\
-0.00054 \\
\end{array}$ & $\begin{array}{l}+0.00024 \\
-0.00024 \\
\end{array}$ & $\begin{array}{l}+0.00002 \\
-0.00005 \\
\end{array}$ & $0.966 \pm 0.028$ \\
\hline
\end{tabular}


TABLE V. NLO pQCD cross section prediction for the production of an isolated photon in the pseudorapidity range $0.00 \leq$ $\left|\eta^{\gamma}\right|<1.37$ in association with a jet in the rapidity range $2.8 \leq\left|y^{\text {jet }}\right|<4.4$ and $p_{\mathrm{T}}^{\text {jet }}>20 \mathrm{GeV}\left(\eta^{\gamma} y^{\text {jet }} \geq 0\right)$. The NLO pQCD cross section has been computed with JETPHOX 1.3 using CT10 PDFs. Details on the calculation of the uncertainties are discussed in Sec. VIII. In the last column the nonperturbative correction factor that must multiply the JETPHOX cross section is shown, with its uncertainty.

\begin{tabular}{|c|c|c|c|c|c|c|c|}
\hline $\begin{array}{l}E_{\mathrm{T}}^{\gamma} \min \\
{[\mathrm{GeV}]}\end{array}$ & $\begin{array}{c}E_{\mathrm{T}}^{\gamma} \max \\
{[\mathrm{GeV}]}\end{array}$ & $\begin{array}{c}\frac{d \sigma}{d E^{\gamma}} \\
{[\mathrm{pb} / \mathrm{GeV}]}\end{array}$ & $\begin{array}{l}\text { stat uncertainty } \\
{[\mathrm{pb} / \mathrm{GeV}]}\end{array}$ & $\begin{array}{l}\text { scale uncertainty } \\
{[\mathrm{pb} / \mathrm{GeV}]}\end{array}$ & $\begin{array}{l}\text { PDF uncertainty } \\
{[\mathrm{pb} / \mathrm{GeV}]}\end{array}$ & $\begin{array}{l}\text { isolation uncertainty } \\
{[\mathrm{pb} / \mathrm{GeV}]}\end{array}$ & $\begin{array}{l}\text { correction } \\
\text { factor }\end{array}$ \\
\hline 25 & 30 & 91 & \pm 2 & $\begin{array}{l}+18 \\
-5\end{array}$ & $\begin{array}{l}+2 \\
-3\end{array}$ & $\begin{array}{l}+10 \\
-0\end{array}$ & $0.904 \pm 0.062$ \\
\hline 30 & 35 & 47 & \pm 1 & $\begin{array}{l}+12 \\
-4\end{array}$ & $\begin{array}{l}+1 \\
-2\end{array}$ & $\begin{array}{l}+5 \\
-0\end{array}$ & $0.919 \pm 0.071$ \\
\hline 35 & 45 & 19.8 & \pm 0.3 & $\begin{array}{l}+4.1 \\
-1.3\end{array}$ & $\begin{array}{l}+0.4 \\
-0.7\end{array}$ & $\begin{array}{l}+1.4 \\
-0.0\end{array}$ & $0.959 \pm 0.035$ \\
\hline 45 & 55 & 6.14 & \pm 0.11 & $\begin{array}{l}+2.31 \\
-0.82\end{array}$ & $\begin{array}{l}+0.18 \\
-0.23\end{array}$ & $\begin{array}{l}+0.59 \\
-0.00\end{array}$ & $0.950 \pm 0.068$ \\
\hline 55 & 70 & 1.97 & \pm 0.04 & $\begin{array}{l}+0.38 \\
-0.22\end{array}$ & $\begin{array}{l}+0.07 \\
-0.09\end{array}$ & $\begin{array}{l}+0.09 \\
-0.04\end{array}$ & $0.960 \pm 0.066$ \\
\hline 70 & 85 & 0.556 & \pm 0.013 & $\begin{array}{l}+0.147 \\
-0.051\end{array}$ & $\begin{array}{l}+0.026 \\
-0.024\end{array}$ & $\begin{array}{l}+0.009 \\
-0.002\end{array}$ & $0.975 \pm 0.067$ \\
\hline 85 & 100 & 0.204 & \pm 0.005 & $\begin{array}{l}+0.049 \\
-0.022\end{array}$ & $\begin{array}{l}+0.012 \\
-0.009\end{array}$ & $\begin{array}{l}+0.010 \\
{ }_{-0.003}\end{array}$ & $0.973 \pm 0.079$ \\
\hline 100 & 125 & 0.064 & \pm 0.002 & $\begin{array}{l}+0.008 \\
-0.011\end{array}$ & $\begin{array}{l}+0.004 \\
-0.003\end{array}$ & $\begin{array}{l}+0.003 \\
-0.000\end{array}$ & $0.973 \pm 0.056$ \\
\hline 125 & 150 & 0.0146 & \pm 0.0005 & $\begin{array}{l}+0.0019 \\
-0.0017\end{array}$ & $\begin{array}{l}+0.0014 \\
-0.0008\end{array}$ & $\begin{array}{l}+0.0012 \\
-0.0004\end{array}$ & $0.979 \pm 0.068$ \\
\hline 150 & 200 & 0.0027 & \pm 0.0001 & $\begin{array}{l}+0.0007 \\
-0.0005 \\
\end{array}$ & $\begin{array}{l}+0.0004 \\
-0.0002 \\
\end{array}$ & $\begin{array}{l}+0.0004 \\
-0.0000\end{array}$ & $1.004 \pm 0.056$ \\
\hline
\end{tabular}

TABLE VI. NLO pQCD cross section prediction for the production of an isolated photon in the pseudorapidity range $0.00 \leq$ $\left|\eta^{\gamma}\right|<1.37$ in association with a jet in the rapidity range $2.8 \leq\left|y^{\text {jet }}\right|<4.4$ and $p_{\mathrm{T}}^{\text {jet }}>20 \mathrm{GeV}\left(\eta^{\gamma} y^{\text {jet }}<0\right)$. The NLO pQCD cross section has been computed with JETPHOX 1.3 using CT10 PDFs. Details on the calculation of the uncertainties are discussed in Sec. VIII. In the last column the nonperturbative correction factor that must multiply the JETPHOX cross section is shown, with its uncertainty.

\begin{tabular}{|c|c|c|c|c|c|c|c|}
\hline $\begin{array}{l}E_{\mathrm{T}}^{\gamma} \min \\
{[\mathrm{GeV}]}\end{array}$ & $\begin{array}{c}E_{\mathrm{T}}^{\gamma} \max \\
{[\mathrm{GeV}]}\end{array}$ & $\begin{array}{c}\frac{d \sigma}{d E_{\gamma}^{\gamma}} \\
{[\mathrm{pb} / \mathrm{GeV}]}\end{array}$ & $\begin{array}{l}\text { stat uncertainty } \\
{[\mathrm{pb} / \mathrm{GeV}]}\end{array}$ & $\begin{array}{l}\text { scale uncertainty } \\
{[\mathrm{pb} / \mathrm{GeV}]}\end{array}$ & $\begin{array}{l}\text { PDF uncertainty } \\
{[\mathrm{pb} / \mathrm{GeV}]}\end{array}$ & $\begin{array}{l}\text { isolation uncertainty } \\
\qquad[\mathrm{pb} / \mathrm{GeV}]\end{array}$ & $\begin{array}{l}\text { correction } \\
\text { factor }\end{array}$ \\
\hline 25 & 30 & 53 & \pm 1 & $\begin{array}{c}+17 \\
-8\end{array}$ & $\begin{array}{l}+1 \\
-1\end{array}$ & $\begin{array}{l}+0 \\
-1\end{array}$ & $0.84 \pm 0.26$ \\
\hline 30 & 35 & 27 & \pm 0 & $\begin{array}{l}+7 \\
-5\end{array}$ & $\begin{array}{l}+1 \\
-1\end{array}$ & $\begin{array}{l}+1 \\
-0\end{array}$ & $0.81 \pm 0.21$ \\
\hline 35 & 45 & 10.4 & \pm 0.2 & $\begin{array}{l}+3.5 \\
-1.2\end{array}$ & $\begin{array}{l}+0.3 \\
-0.3\end{array}$ & $\begin{array}{l}+0.5 \\
-0.0\end{array}$ & $0.92 \pm 0.09$ \\
\hline 45 & 55 & 3.37 & \pm 0.05 & $\begin{array}{l}+0.88 \\
-0.69\end{array}$ & $\begin{array}{l}+0.12 \\
-0.12\end{array}$ & $\begin{array}{l}+0.23 \\
-0.00\end{array}$ & $0.88 \pm 0.08$ \\
\hline 55 & 70 & 1.00 & \pm 0.02 & $\begin{array}{l}+0.30 \\
-0.21\end{array}$ & $\begin{array}{l}+0.04 \\
-0.04\end{array}$ & $\begin{array}{l}+0.10 \\
-0.00\end{array}$ & $0.93 \pm 0.15$ \\
\hline 70 & 85 & 0.287 & \pm 0.005 & $\begin{array}{l}+0.094 \\
-0.058\end{array}$ & $\begin{array}{l}+0.017 \\
-0.014\end{array}$ & $\begin{array}{l}+0.005 \\
-0.002\end{array}$ & $0.95 \pm 0.06$ \\
\hline 85 & 100 & 0.091 & \pm 0.002 & $\begin{array}{l}+0.035 \\
-0.010\end{array}$ & $\begin{array}{l}+0.007 \\
-0.005\end{array}$ & $\begin{array}{l}+0.004 \\
-0.000\end{array}$ & $0.97 \pm 0.10$ \\
\hline 100 & 125 & 0.028 & \pm 0.001 & $\begin{array}{l}+0.010 \\
-0.006\end{array}$ & $\begin{array}{l}+0.003 \\
-0.002\end{array}$ & $\begin{array}{l}+0.000 \\
-0.001\end{array}$ & $0.94 \pm 0.12$ \\
\hline 125 & 150 & 0.0067 & \pm 0.0002 & $\begin{array}{l}+0.0030 \\
-0.0016\end{array}$ & $\begin{array}{l}+0.0008 \\
-0.0005\end{array}$ & $\begin{array}{l}+0.0000 \\
-0.0000\end{array}$ & $1.00 \pm 0.11$ \\
\hline 150 & 200 & 0.0014 & \pm 0.0001 & $\begin{array}{l}+0.0004 \\
-0.0004 \\
\end{array}$ & $\begin{array}{l}+0.0002 \\
-0.0001 \\
\end{array}$ & $\begin{array}{l}+0.0000 \\
-0.0001 \\
\end{array}$ & $0.92 \pm 0.21$ \\
\hline
\end{tabular}




\section{APPENDIX B: MEASURED photon + jet CROSS SECTION}

Tables VII, VIII, IX, X, XI, and XII show the measured photon + jet differential cross sections, in the six photon-jet angular configurations under study, and the comparison to the theoretical predictions.

TABLE VII. Measured cross section as a function of the photon transverse energy, $E_{\mathrm{T}}^{\gamma}$, for $\left|\eta^{\gamma}\right| \leq 1.37,\left|y^{\mathrm{jet}}\right|<1.2$ and $\eta^{\gamma} y^{\mathrm{jet}} \geq 0$. The last two columns show the cross section predicted by JETPHOX and multiplied by the corresponding nonperturbative correction factor, and its uncertainty.

\begin{tabular}{|c|c|c|c|c|c|c|c|}
\hline \multirow[b]{2}{*}{$\begin{array}{l}E_{\mathrm{T}}^{\gamma} \min \\
{[\mathrm{GeV}]}\end{array}$} & \multirow[b]{2}{*}{$\begin{array}{c}E_{\mathrm{T}}^{\gamma} \max \\
{[\mathrm{GeV}]}\end{array}$} & \multicolumn{4}{|c|}{ Measured cross section } & \multicolumn{2}{|c|}{ Predicted cross section } \\
\hline & & $\begin{array}{c}\frac{d \sigma}{d E_{\gamma}^{\gamma}} \\
{[\mathrm{pb} / \mathrm{GeV}]}\end{array}$ & $\begin{array}{c}\text { stat } \\
{[\mathrm{pb} / \mathrm{GeV}]}\end{array}$ & $\begin{array}{c}\text { syst } \\
{[\mathrm{pb} / \mathrm{GeV}]}\end{array}$ & $\begin{array}{l}\text { total exp. uncertainty } \\
{[\mathrm{pb} / \mathrm{GeV}]}\end{array}$ & $\begin{array}{c}\frac{d \sigma}{d E_{j}^{\gamma}} \\
{[\mathrm{pb} / \mathrm{GeV}]}\end{array}$ & $\begin{array}{l}\text { total theory uncertainty } \\
{[\mathrm{pb} / \mathrm{GeV}]}\end{array}$ \\
\hline 25 & 30 & 394 & \pm 8 & $\begin{array}{l}+74 \\
-30\end{array}$ & $\begin{array}{l}+74 \\
-31\end{array}$ & 510 & $\begin{array}{l}+51 \\
-55\end{array}$ \\
\hline 30 & 35 & 258 & \pm 6 & $\begin{array}{r}+49 \\
-23\end{array}$ & $\begin{array}{r}+1 \\
+50 \\
-23\end{array}$ & 315 & $\begin{array}{r}+52 \\
-29\end{array}$ \\
\hline 35 & 45 & 137 & \pm 3 & $\begin{array}{l}+27 \\
-13\end{array}$ & $\begin{array}{l}+27 \\
-13\end{array}$ & 153 & $\begin{array}{l}+16 \\
-16\end{array}$ \\
\hline 45 & 55 & 60.9 & \pm 0.7 & $\begin{array}{l}+7.0 \\
-5.2\end{array}$ & $\begin{array}{l}+7.1 \\
-5.2\end{array}$ & 59.9 & $\begin{array}{l}+7.2 \\
-4.5\end{array}$ \\
\hline 55 & 70 & 24.8 & \pm 0.3 & $\begin{array}{l}+3.0 \\
-2.3\end{array}$ & $\begin{array}{l}+3.0 \\
-2.4\end{array}$ & 24.3 & $\begin{array}{l}+2.5 \\
-2.0\end{array}$ \\
\hline 70 & 85 & 9.51 & \pm 0.20 & $\begin{array}{l}+1.22 \\
-0.98\end{array}$ & $\begin{array}{l}+1.24 \\
-1.00\end{array}$ & 9.21 & $\begin{array}{l}+1.01 \\
-0.95\end{array}$ \\
\hline 85 & 100 & 4.40 & \pm 0.15 & $\begin{array}{l}+0.55 \\
-0.48\end{array}$ & $\begin{array}{l}+0.57 \\
-0.50\end{array}$ & 4.21 & $\begin{array}{l}+0.41 \\
-0.39\end{array}$ \\
\hline 100 & 125 & 1.77 & \pm 0.07 & $\begin{array}{l}+0.23 \\
-0.20\end{array}$ & $\begin{array}{l}+0.24 \\
-0.22\end{array}$ & 1.76 & $\begin{array}{l}+0.17 \\
-0.15\end{array}$ \\
\hline 125 & 150 & 0.698 & \pm 0.038 & $\begin{array}{l}+0.096 \\
-0.085\end{array}$ & $\begin{array}{l}+0.103 \\
-0.093\end{array}$ & 0.693 & $\begin{array}{l}+0.065 \\
-0.061\end{array}$ \\
\hline 150 & 200 & 0.226 & \pm 0.017 & $\begin{array}{l}+0.032 \\
-0.029\end{array}$ & $\begin{array}{l}+0.036 \\
-0.034\end{array}$ & 0.236 & $\begin{array}{l}+0.025 \\
-0.020\end{array}$ \\
\hline 200 & 400 & 0.0283 & \pm 0.0028 & $\begin{array}{l}+0.0041 \\
-0.0038 \\
\end{array}$ & $\begin{array}{l}+0.0050 \\
-0.0048 \\
\end{array}$ & 0.0263 & $\begin{array}{l}+0.0027 \\
-0.0025 \\
\end{array}$ \\
\hline
\end{tabular}

TABLE VIII. Measured cross section as a function of the photon transverse energy, $E_{\mathrm{T}}^{\gamma}$, for $\left|\eta^{\gamma}\right| \leq 1.37,\left|y^{j}\right|<1.2$ and $\eta^{\gamma} y^{\text {jet }}<0$. The last two columns show the cross section predicted by JETPHOX and multiplied by the corresponding nonperturbative correction factor, and its uncertainty.

\begin{tabular}{|c|c|c|c|c|c|c|c|}
\hline \multirow[b]{2}{*}{$\begin{array}{l}E_{\mathrm{T}}^{\gamma} \min \\
{[\mathrm{GeV}]}\end{array}$} & \multirow[b]{2}{*}{$\begin{array}{c}E_{\mathrm{T}}^{\gamma} \max \\
{[\mathrm{GeV}]}\end{array}$} & \multicolumn{4}{|c|}{ Measured cross section } & \multicolumn{2}{|c|}{ Predicted cross section } \\
\hline & & $\begin{array}{c}\frac{d \sigma}{d E^{\gamma}} \\
{[\mathrm{pb} / \mathrm{GeV}]}\end{array}$ & $\begin{array}{c}\text { stat } \\
{[\mathrm{pb} / \mathrm{GeV}]}\end{array}$ & $\begin{array}{c}\text { syst } \\
{[\mathrm{pb} / \mathrm{GeV}]}\end{array}$ & $\begin{array}{l}\text { total exp. uncertainty } \\
\qquad[\mathrm{pb} / \mathrm{GeV}]\end{array}$ & $\begin{array}{c}\frac{d \sigma}{d E^{\gamma}} \\
{[\mathrm{pb} / \mathrm{GeV}]}\end{array}$ & $\begin{array}{l}\text { total theory } \\
\text { uncertainty }[\mathrm{pb} / \mathrm{GeV}]\end{array}$ \\
\hline 25 & 30 & 324 & \pm 7 & $\begin{array}{l}+64 \\
+29\end{array}$ & $\begin{array}{l}+65 \\
-30\end{array}$ & 389 & $\begin{array}{l}+53 \\
-49\end{array}$ \\
\hline 30 & 35 & 201 & \pm 5 & $\begin{array}{l}+41 \\
-20\end{array}$ & $\begin{array}{l}+41 \\
-20\end{array}$ & 246 & $\begin{array}{l}+30 \\
-45\end{array}$ \\
\hline 35 & 45 & 112 & \pm 3 & $\begin{array}{l}+23 \\
-12\end{array}$ & $\begin{array}{l}+23 \\
-12\end{array}$ & 116 & $\begin{array}{l}+18 \\
-17\end{array}$ \\
\hline 45 & 55 & 45.5 & \pm 0.5 & $\begin{array}{l}+5.6 \\
-3.9\end{array}$ & $\begin{array}{l}+5.6 \\
-3.9\end{array}$ & 46.0 & $\begin{array}{l}+5.1 \\
-6.9\end{array}$ \\
\hline 55 & 70 & 18.3 & \pm 0.3 & $\begin{array}{l}+2.4 \\
-1.7\end{array}$ & $\begin{array}{l}+2.4 \\
-1.8\end{array}$ & 16.9 & $\begin{array}{l}+2.9 \\
-1.8\end{array}$ \\
\hline 70 & 85 & 7.18 & \pm 0.18 & $\begin{array}{l}+0.97 \\
-0.74\end{array}$ & $\begin{array}{l}+0.99 \\
-0.76\end{array}$ & 6.68 & $\begin{array}{l}+0.69 \\
-0.79\end{array}$ \\
\hline 85 & 100 & 3.26 & \pm 0.14 & $\begin{aligned}+0.38 \\
-0.36\end{aligned}$ & $\begin{array}{l}+0.40 \\
-0.38\end{array}$ & 2.87 & $\begin{array}{l}+0.36 \\
-0.28\end{array}$ \\
\hline 100 & 125 & 1.36 & \pm 0.05 & $\begin{array}{l}+0.17 \\
-0.16\end{array}$ & $\begin{array}{l}+0.17 \\
-0.17\end{array}$ & 1.22 & $\begin{array}{l}+0.15 \\
-0.13\end{array}$ \\
\hline 125 & 150 & 0.503 & \pm 0.037 & $\begin{array}{l}+0.065 \\
-0.062\end{array}$ & $\begin{array}{l}+0.075 \\
-0.072\end{array}$ & 0.466 & $\begin{array}{l}+0.056 \\
-0.044\end{array}$ \\
\hline 150 & 200 & 0.156 & \pm 0.014 & $\begin{array}{l}+0.023 \\
-0.020\end{array}$ & $\begin{array}{l}+0.027 \\
-0.025\end{array}$ & 0.156 & $\begin{array}{l}+0.021 \\
-0.016\end{array}$ \\
\hline 200 & 400 & 0.0182 & \pm 0.0022 & $\begin{array}{l}+0.0028 \\
-0.0025 \\
\end{array}$ & $\begin{array}{l}+0.0035 \\
-0.0033 \\
\end{array}$ & 0.0167 & $\begin{array}{l}+0.0019 \\
-0.0017 \\
\end{array}$ \\
\hline
\end{tabular}


TABLE IX. Measured cross section as a function of the photon transverse energy, $E_{\mathrm{T}}^{\gamma}$, for $\left|\eta^{\gamma}\right| \leq 1.37,1.2 \leq\left|y^{\text {jet }}\right|<2.8$ and $\eta^{\gamma} y^{\text {jet }} \geq 0$. The last two columns show the cross section predicted by JETPHOX and multiplied by the corresponding nonperturbative correction factor, and its uncertainty.

\begin{tabular}{|c|c|c|c|c|c|c|c|}
\hline \multirow[b]{2}{*}{$\begin{array}{l}E_{\mathrm{T}}^{\gamma} \min \\
{[\mathrm{GeV}]}\end{array}$} & \multirow[b]{2}{*}{$\begin{array}{c}E_{\mathrm{T}}^{\gamma} \max \\
{[\mathrm{GeV}]}\end{array}$} & \multicolumn{4}{|c|}{ Measured cross section } & \multicolumn{2}{|c|}{ Predicted cross section } \\
\hline & & {$\left[\begin{array}{c}\frac{d \sigma}{d E^{\gamma}} \\
{[\mathrm{pb} / \mathrm{GeV}]}\end{array}\right.$} & $\begin{array}{c}\text { stat } \\
{[\mathrm{pb} / \mathrm{GeV}]}\end{array}$ & $\begin{array}{c}\text { syst } \\
{[\mathrm{pb} / \mathrm{GeV}]}\end{array}$ & $\begin{array}{l}\text { total exp. uncertainty } \\
{[\mathrm{pb} / \mathrm{GeV}]}\end{array}$ & $\begin{array}{c}\frac{d \sigma}{d E^{\gamma}} \\
{[\mathrm{pb} / \mathrm{GeV}]}\end{array}$ & $\begin{array}{l}\text { total theory uncertainty } \\
\qquad[\mathrm{pb} / \mathrm{GeV}]\end{array}$ \\
\hline 25 & 30 & 316 & \pm 7 & $\begin{array}{l}+54 \\
-30\end{array}$ & $\begin{array}{l}+55 \\
-31\end{array}$ & 401 & $\begin{array}{l}+53 \\
+46\end{array}$ \\
\hline 30 & 35 & 210 & \pm 6 & $\begin{array}{l}+37 \\
-22\end{array}$ & $\begin{array}{l}+37 \\
-23\end{array}$ & 247 & $\begin{array}{l}+34 \\
+32\end{array}$ \\
\hline 35 & 45 & 105 & \pm 2 & $\begin{array}{l}+19 \\
-12\end{array}$ & $\begin{array}{l}+19 \\
-12\end{array}$ & 119 & $\begin{array}{l}+17 \\
-16\end{array}$ \\
\hline 45 & 55 & 43.6 & \pm 0.6 & $\begin{array}{l}+5.0 \\
-3.7\end{array}$ & $\begin{array}{l}+5.1 \\
-3.8\end{array}$ & 42.1 & $\begin{array}{l}+6.7 \\
+4.2\end{array}$ \\
\hline 55 & 70 & 17.5 & \pm 0.3 & $\begin{array}{l}+2.1 \\
-1.7\end{array}$ & $\begin{array}{l}5.0 \\
+2.2 \\
-1.7\end{array}$ & 16.6 & $\begin{array}{l}-2.2 \\
+2.1\end{array}$ \\
\hline 70 & 85 & 6.39 & \pm 0.17 & $\begin{array}{l}+0.82 \\
-0.66\end{array}$ & $\begin{array}{l}+0.84 \\
-0.68\end{array}$ & 5.99 & $\begin{array}{l}+0.70 \\
-0.58\end{array}$ \\
\hline 85 & 100 & 2.71 & \pm 0.10 & $\begin{array}{l}+0.35 \\
-0.29\end{array}$ & $\begin{array}{l}+0.36 \\
-0.31\end{array}$ & 2.63 & $\begin{array}{l}+0.30 \\
-0.25\end{array}$ \\
\hline 100 & 125 & 1.27 & \pm 0.05 & $\begin{array}{l}+0.17 \\
-0.15\end{array}$ & $\begin{array}{l}+0.18 \\
-0.16\end{array}$ & 1.08 & $\begin{array}{l}+0.12 \\
-0.10\end{array}$ \\
\hline 125 & 150 & 0.441 & \pm 0.028 & $\begin{array}{l}+0.062 \\
-0.054\end{array}$ & $\begin{array}{l}+0.068 \\
-0.061\end{array}$ & 0.400 & $\begin{array}{l}+0.052 \\
-0.037\end{array}$ \\
\hline 150 & 200 & 0.102 & \pm 0.012 & $\begin{array}{l}+0.015 \\
-0.013\end{array}$ & $\begin{array}{l}+0.019 \\
-0.018\end{array}$ & 0.125 & $\begin{array}{l}+0.010 \\
-0.012\end{array}$ \\
\hline 200 & 400 & 0.0090 & \pm 0.0017 & $\begin{array}{l}+0.0013 \\
-0.0012\end{array}$ & $\begin{array}{l}+0.0022 \\
-0.0021\end{array}$ & 0.0117 & $\begin{array}{l}+0.0001 \\
-0.0012\end{array}$ \\
\hline
\end{tabular}

TABLE X. Measured cross section as a function of the photon transverse energy, $E_{\mathrm{T}}^{\gamma}$, for $\left|\eta^{\gamma}\right| \leq 1.37,1.2 \leq\left|y^{\text {jet }}\right|<2.8$ and $\eta^{\gamma} y^{\text {jet }}<0$. The last two columns show the cross section predicted by JETPHOX and multiplied by the corresponding nonperturbative correction factor, and its uncertainty.

\begin{tabular}{|c|c|c|c|c|c|c|c|}
\hline \multirow[b]{2}{*}{$\begin{array}{l}E_{\mathrm{T}}^{\gamma} \min \\
{[\mathrm{GeV}]}\end{array}$} & \multirow[b]{2}{*}{$\begin{array}{c}E_{\mathrm{T}}^{\gamma} \max \\
{[\mathrm{GeV}]}\end{array}$} & \multicolumn{4}{|c|}{ Measured cross section } & \multicolumn{2}{|c|}{ Predicted cross section } \\
\hline & & $\begin{array}{c}\frac{d \sigma}{d E^{\gamma}} \\
{[\mathrm{pb} / \mathrm{GeV}]}\end{array}$ & $\begin{array}{c}\text { stat } \\
{[\mathrm{pb} / \mathrm{GeV}]}\end{array}$ & $\begin{array}{c}\text { syst } \\
{[\mathrm{pb} / \mathrm{GeV}]}\end{array}$ & $\begin{array}{l}\text { total exp. uncertainty } \\
\qquad[\mathrm{pb} / \mathrm{GeV}]\end{array}$ & $\begin{array}{c}\frac{d \sigma}{d E^{\gamma}} \\
{[\mathrm{pb} / \mathrm{GeV}]}\end{array}$ & $\begin{array}{l}\text { total theory uncertainty } \\
\qquad[\mathrm{pb} / \mathrm{GeV}]\end{array}$ \\
\hline 25 & 30 & 188 & \pm 6 & $\begin{array}{l}+35 \\
-27\end{array}$ & $\begin{array}{l}+36 \\
-27\end{array}$ & 243 & $\begin{array}{l}+38 \\
-49\end{array}$ \\
\hline 30 & 35 & 115 & \pm 4 & $\begin{array}{l}+22 \\
-17\end{array}$ & $\begin{array}{l}+23 \\
-18\end{array}$ & 128 & $\begin{array}{l}+24 \\
-23\end{array}$ \\
\hline 35 & 45 & 58 & \pm 2 & $\begin{array}{l}+11 \\
-9\end{array}$ & $\begin{array}{l}10 \\
+12 \\
-9\end{array}$ & 58 & $\begin{array}{l}+12 \\
+9\end{array}$ \\
\hline 45 & 55 & 24.1 & \pm 0.5 & $\begin{array}{l}+3.1 \\
-2.1\end{array}$ & $\begin{array}{l}+3.1 \\
-2.2\end{array}$ & 21.5 & $\begin{array}{l}+3.9 \\
-4.2\end{array}$ \\
\hline 55 & 70 & 8.8 & \pm 0.2 & $\begin{array}{l}+1.2 \\
-0.9\end{array}$ & $\begin{array}{l}+1.2 \\
-0.9\end{array}$ & 7.8 & $\begin{array}{l}+1.5 \\
-1.2\end{array}$ \\
\hline 70 & 85 & 3.32 & \pm 0.11 & $\begin{array}{l}+0.46 \\
-0.35\end{array}$ & $\begin{array}{l}+0.48 \\
-0.37\end{array}$ & 2.76 & $\begin{array}{l}+0.41 \\
-0.46\end{array}$ \\
\hline 85 & 100 & 1.49 & \pm 0.09 & $\begin{array}{l}+0.16 \\
-0.16\end{array}$ & $\begin{array}{r}+0.19 \\
-0.18\end{array}$ & 1.14 & $\begin{array}{l}+0.25 \\
-0.19\end{array}$ \\
\hline 100 & 125 & 0.54 & \pm 0.04 & $\begin{array}{l}+0.06 \\
-0.06\end{array}$ & $\begin{array}{l}+0.07 \\
-0.07\end{array}$ & 0.44 & $\begin{array}{l}+0.08 \\
-0.07\end{array}$ \\
\hline 125 & 150 & 0.175 & \pm 0.022 & $\begin{array}{l}+0.022 \\
-0.022\end{array}$ & $\begin{array}{l}+0.031 \\
-0.031\end{array}$ & 0.154 & $\begin{array}{l}+0.040 \\
-0.021\end{array}$ \\
\hline 150 & 200 & 0.055 & \pm 0.008 & $\begin{array}{l}+0.007 \\
-0.008\end{array}$ & $\begin{array}{l}+0.011 \\
-0.011\end{array}$ & 0.047 & $\begin{array}{l}+0.009 \\
-0.008\end{array}$ \\
\hline 200 & 400 & 0.0041 & \pm 0.0010 & $\begin{array}{r}+0.0006 \\
-0.0006 \\
\end{array}$ & $\begin{array}{l}+0.0011 \\
-0.0012 \\
\end{array}$ & 0.0041 & $\begin{array}{l}+0.0010 \\
+0.0006\end{array}$ \\
\hline
\end{tabular}


TABLE XI. Measured cross section as a function of the photon transverse energy, $E_{\mathrm{T}}^{\gamma}$, for $\left|\eta^{\gamma}\right| \leq 1.37,2.8 \leq\left|y^{\text {jet }}\right|<4.4$ and $\eta^{\gamma} y^{\text {jet }} \geq 0$. The last two columns show the cross section predicted by JETPHOX and multiplied by the corresponding nonperturbative correction factor, and its uncertainty.

\begin{tabular}{|c|c|c|c|c|c|c|c|}
\hline \multirow[b]{2}{*}{$\begin{array}{l}E_{\mathrm{T}}^{\gamma} \min \\
{[\mathrm{GeV}]}\end{array}$} & \multirow[b]{2}{*}{$\begin{array}{c}E_{\mathrm{T}}^{\gamma} \max \\
{[\mathrm{GeV}]}\end{array}$} & \multicolumn{4}{|c|}{ Measured cross section } & \multicolumn{2}{|c|}{ Predicted cross section } \\
\hline & & {$\left[\begin{array}{c}\frac{d \sigma}{d E^{\gamma}} \\
{[\mathrm{pb} / \mathrm{GeV}]}\end{array}\right.$} & $\begin{array}{c}\text { stat } \\
{[\mathrm{pb} / \mathrm{GeV}]}\end{array}$ & $\begin{array}{c}\text { syst } \\
{[\mathrm{pb} / \mathrm{GeV}]}\end{array}$ & $\begin{array}{l}\text { total exp. uncertainty } \\
{[\mathrm{pb} / \mathrm{GeV}]}\end{array}$ & {$\left[\begin{array}{c}\frac{d \sigma}{d E^{\gamma}} \\
{[\mathrm{pb} / \mathrm{GeV}]}\end{array}\right.$} & $\begin{array}{l}\text { total theory uncertainty } \\
\qquad[\mathrm{pb} / \mathrm{GeV}]\end{array}$ \\
\hline 25 & 30 & 66 & \pm 4 & $\begin{array}{l}+18 \\
-9\end{array}$ & $\begin{array}{l}+19 \\
-10\end{array}$ & 82 & $\begin{array}{c}+20 \\
-8\end{array}$ \\
\hline 30 & 35 & 46 & \pm 3 & $\begin{array}{l}+13 \\
-7\end{array}$ & $\begin{array}{l}+13 \\
-8\end{array}$ & 43 & $\begin{array}{l}+12 \\
-5\end{array}$ \\
\hline 35 & 45 & 20 & \pm 1 & $\begin{array}{l}+6 \\
-3\end{array}$ & $\begin{array}{l}+6 \\
-3\end{array}$ & 19 & $\begin{array}{l}+4 \\
-2\end{array}$ \\
\hline 45 & 55 & 8.1 & \pm 0.3 & $\begin{array}{l}+1.4 \\
-0.8\end{array}$ & $\begin{array}{l}+1.4 \\
-0.8\end{array}$ & 5.8 & $\begin{array}{l}+2.3 \\
-0.9\end{array}$ \\
\hline 55 & 70 & 2.4 & \pm 0.1 & $\begin{array}{l}+0.4 \\
-0.2\end{array}$ & $\begin{array}{l}+0.4 \\
-0.3\end{array}$ & 1.9 & $\begin{array}{l}+0.4 \\
-0.3\end{array}$ \\
\hline 70 & 85 & 0.86 & \pm 0.06 & $\begin{array}{l}+0.15 \\
-0.10\end{array}$ & $\begin{array}{l}+0.17 \\
-0.11\end{array}$ & 0.54 & $\begin{array}{l}+0.15 \\
-0.07\end{array}$ \\
\hline 85 & 100 & 0.24 & \pm 0.03 & $\begin{array}{l}+0.03 \\
-0.03\end{array}$ & $\begin{array}{l}+0.04 \\
-0.04\end{array}$ & 0.20 & $\begin{array}{l}+0.05 \\
-0.03\end{array}$ \\
\hline 100 & 125 & 0.07 & \pm 0.01 & $\begin{array}{l}+0.01 \\
-0.01\end{array}$ & $\begin{array}{l}+0.02 \\
-0.02\end{array}$ & 0.06 & $\begin{array}{l}+0.01 \\
-0.01\end{array}$ \\
\hline 125 & 150 & 0.014 & \pm 0.007 & $\begin{array}{l}+0.002 \\
-0.002\end{array}$ & $\begin{array}{l}+0.007 \\
-0.007\end{array}$ & 0.014 & $\begin{array}{l}+0.003 \\
-0.002\end{array}$ \\
\hline 150 & 200 & 0.0028 & \pm 0.0019 & $\begin{array}{l}+0.0004 \\
-0.0004\end{array}$ & $\begin{array}{l}+0.0019 \\
-0.0020\end{array}$ & 0.0027 & $\begin{array}{l}+0.0009 \\
-0.0006\end{array}$ \\
\hline
\end{tabular}

TABLE XII. Measured cross section as a function of the photon transverse energy, $E_{\mathrm{T}}^{\gamma}$, for $\left|\eta^{\gamma}\right| \leq 1.37,2.8 \leq\left|y^{\text {jet }}\right|<4.4$ and $\eta^{\gamma} y^{\text {jet }}<0$. The last two columns show the cross section predicted by JETPHOX and multiplied by the corresponding nonperturbative correction factor, and its uncertainty.

\begin{tabular}{|c|c|c|c|c|c|c|c|}
\hline \multirow[b]{2}{*}{$\begin{array}{l}E_{\mathrm{T}}^{\gamma} \min \\
{[\mathrm{GeV}]}\end{array}$} & \multirow[b]{2}{*}{$\begin{array}{c}E_{\mathrm{T}}^{\gamma} \max \\
{[\mathrm{GeV}]}\end{array}$} & \multicolumn{4}{|c|}{ Measured cross section } & \multicolumn{2}{|c|}{ Predicted cross section } \\
\hline & & $\begin{array}{c}\frac{d \sigma}{d E_{\mathrm{\gamma}}^{\gamma}} \\
{[\mathrm{pb} / \mathrm{GeV}]}\end{array}$ & $\begin{array}{c}\text { stat } \\
{[\mathrm{pb} / \mathrm{GeV}]}\end{array}$ & $\begin{array}{c}\text { syst } \\
{[\mathrm{pb} / \mathrm{GeV}]}\end{array}$ & $\begin{array}{l}\text { total exp. uncertainty } \\
{[\mathrm{pb} / \mathrm{GeV}]}\end{array}$ & $\begin{array}{c}\frac{d \sigma}{d E_{\mathrm{\gamma}}^{\gamma}} \\
{[\mathrm{pb} / \mathrm{GeV}]}\end{array}$ & $\begin{array}{l}\text { total theory uncertainty } \\
\qquad[\mathrm{pb} / \mathrm{GeV}]\end{array}$ \\
\hline 25 & 30 & 31 & \pm 4 & $\begin{array}{l}+12 \\
-4\end{array}$ & $\begin{array}{l}+13 \\
-6\end{array}$ & 44 & $\begin{array}{r}+19 \\
-14\end{array}$ \\
\hline 30 & 35 & 21 & \pm 2 & $\begin{array}{l}+8 \\
-3\end{array}$ & $\begin{array}{l}+9 \\
-4\end{array}$ & 22 & $\begin{array}{l}+8 \\
-6\end{array}$ \\
\hline 35 & 45 & 12 & \pm 1 & $\begin{array}{l}+5 \\
-2\end{array}$ & $\begin{array}{l}+5 \\
-2\end{array}$ & 10 & $\begin{array}{l}+3 \\
-1\end{array}$ \\
\hline 45 & 55 & 3.5 & \pm 0.2 & $\begin{array}{l}+1.1 \\
-0.6\end{array}$ & $\begin{array}{l}+1.1 \\
-0.6\end{array}$ & 3.0 & $\begin{array}{l}+0.8 \\
-0.7\end{array}$ \\
\hline 55 & 70 & 1.5 & \pm 0.1 & $\begin{array}{l}+0.5 \\
+0.2\end{array}$ & $\begin{array}{l}+0.5 \\
+0.3\end{array}$ & 0.9 & $\begin{array}{l}+0.3 \\
+0.2\end{array}$ \\
\hline 70 & 85 & 0.38 & \pm 0.04 & $\begin{array}{l}+0.11 \\
-0.06\end{array}$ & $\begin{array}{l}+0.12 \\
-0.08\end{array}$ & 0.27 & $\begin{array}{l}+0.09 \\
-0.06\end{array}$ \\
\hline 85 & 100 & 0.12 & \pm 0.02 & $\begin{array}{l}+0.01 \\
-0.01\end{array}$ & $\begin{array}{l}+0.03 \\
-0.03\end{array}$ & 0.09 & $\begin{array}{l}+0.04 \\
-0.01\end{array}$ \\
\hline 100 & 125 & 0.036 & \pm 0.011 & $\begin{array}{l}+0.002 \\
-0.002\end{array}$ & $\begin{array}{l}+0.011 \\
-0.011\end{array}$ & 0.027 & $\begin{array}{l}+0.010 \\
-0.007\end{array}$ \\
\hline 125 & 150 & 0.015 & \pm 0.007 & $\begin{array}{l}+0.002 \\
+0.002\end{array}$ & $\begin{array}{l}+0.007 \\
-0.007\end{array}$ & 0.007 & $\begin{array}{l}+0.003 \\
-0.002\end{array}$ \\
\hline 150 & 200 & 0.0023 & \pm 0.0019 & $\begin{array}{l}+0.0003 \\
-0.0003 \\
\end{array}$ & $\begin{array}{l}+0.0019 \\
-0.0019 \\
\end{array}$ & 0.0013 & $\begin{array}{l}+0.0005 \\
-0.0005 \\
\end{array}$ \\
\hline
\end{tabular}

\section{APPENDIX C: THE ATLAS COLLABORATION}

[1] S. Catani et al., J. High Energy Phys. 05 (2002) 028.

[2] P. Aurenche et al., Phys. Rev. D 73, 094007 (2006).

[3] Z. Belghobsi et al., Phys. Rev. D 79, 114024 (2009).

[4] E. N. Argyres, A. P. Contogouris, N. Mebarki, and S. D. P. Vlassopulos, Phys. Rev. D 35, 1584 (1987).
[5] P. Aurenche, R. Baier, M. Fontannaz, J. F. Owens, and M. Werlen, Phys. Rev. D 39, 3275 (1989).

[6] D. d'Enterria and J. Rojo, Nucl. Phys. B860, 311 (2012).

[7] J. Gallicchio and M. D. Schwartz, J. High Energy Phys. 10 (2011) 103. 
[8] ATLAS Collaboration, Report No. ATLAS-CONF-2011031, 2011, http://cdsweb.cern.ch/record/1337781.

[9] ATLAS Collaboration, Eur. Phys. J. C 71, 1728 (2011).

[10] ATLAS Collaboration, Phys. Rev. D 83, 052005 (2011).

[11] ATLAS Collaboration, Phys. Lett. B 706, 150 (2011).

[12] ATLAS Collaboration, Phys. Rev. D 85, 012003 (2012).

[13] The ATLAS reference system is a Cartesian right-handed coordinate system, with the nominal collision point at the origin. The anticlockwise beam direction defines the positive $z$-axis, while the positive $x$-axis is defined as pointing from the collision point to the center of the LHC ring and the positive $y$-axis points upwards. The azimuthal angle $\phi$ is measured around the beam axis, and the polar angle $\theta$ is measured with respect to the $z$-axis. Pseudorapidity is defined as $\eta=-\ln \tan (\theta / 2)$, and transverse energy is defined as $E_{\mathrm{T}}=E \sin \theta$.

[14] M. Cacciari, G. P. Salam, and G. Soyez, J. High Energy Phys. 04 (2008) 005.

[15] M. Cacciari, G. P. Salam, and S. Sapeta, J. High Energy Phys. 04 (2010) 065.

[16] V. M. Abazov et al. (D0 Collaboration), Phys. Lett. B 666, 435 (2008).

[17] F. Abe et al. (CDF Collaboration), Phys. Rev. D 57, 67 (1998).

[18] T. Akesson et al. (Axial Field Spectrometer Collaboration), Z. Phys. C 34, 293 (1987).

[19] J. Alitti et al. (UA2 Collaboration), Phys. Lett. B 299, 174 (1993).

[20] F. D. Aaron et al. (H1 Collaboration), Eur. Phys. J. C 66, 17 (2010).

[21] F. D. Aaron et al. (H1 Collaboration), Eur. Phys. J. C 54, 371 (2008).

[22] S. Chekanov et al. (ZEUS Collaboration), Eur. Phys. J. C 49, 511 (2007).

[23] S. Chekanov et al. (ZEUS Collaboration), Phys. Lett. B 595, 86 (2004).

[24] ATLAS Collaboration, JINST 3, S08003 (2008).

[25] ATLAS Collaboration, Eur. Phys. J. C 72, 1909 (2012).

[26] ATLAS Collaboration, arXiv:1112.6426 [Eur. Phys. J. C (to be published)].

[27] ATLAS Collaboration, Eur. Phys. J. C 71, 1630 (2011).
[28] ATLAS Collaboration, Report No. ATLAS-CONF-2011011, 2011, http://cdsweb.cern.ch/record/1334563.

[29] T. Sjöstrand, S. Mrenna, and P.Z. Skands, J. High Energy Phys. 05 (2006) 026.

[30] ATLAS Collaboration, Report No. ATLAS-CONF-2010031, 2010, http://cdsweb.cern.ch/record/1277665.

[31] S. Agostinelli et al. (GEANT4), Nucl. Instrum. Methods Phys. Res., Sect. A 506, 250 (2003).

[32] ATLAS Collaboration, Eur. Phys. J. C 70, 8232010.

[33] G. Corcella et al., J. High Energy Phys. 01 (2001) 010.

[34] ATLAS Collaboration, Report No. ATL-PHYS-PUB2010-014, 2010, http://cdsweb.cern.ch/record/1303025.

[35] J. M. Butterworth, J. R. Forshaw, and M. H. Seymour, Z. Phys. C 72, 637 (1996).

[36] ATLAS Collaboration, arXiv:0901.0512.

[37] ATLAS Collaboration, ATL-PHYS-PUB-2011-007, 2011, http://cdsweb.cern.ch/record/1345329.

[38] M. Cacciari, G. P. Salam, and G. Soyez, J. High Energy Phys. 04 (2008) 063.

[39] G. D’Agostini, Nucl. Instrum. Methods Phys. Res., Sect. A 362, 487 (1995).

[40] See Supplemental Material at URL for tables detailing the uncertainties for each $E_{\mathrm{T}}^{\gamma}$ bin and each angular configuration.

[41] ATLAS Collaboration, arXiv:1112.6297 [Phys. Rev. D (to be published)].

[42] ATLAS Collaboration, arXiv:1201.1276 [Phys. Rev. D (to be published)].

[43] ATLAS Collaboration, Report No. ATLAS-CONF-2011013, 2011, http://cdsweb.cern.ch/record/1334838.

[44] ATLAS Collaboration, arXiv:1109.5141 [Phys. Rev. D (to be published)].

[45] L. Bourhis, M. Fontannaz, J. P. Guillet, and M. Werlen, Eur. Phys. J. C 19, 89 (2001).

[46] J. Pumplin et al., J. High Energy Phys. 07 (2002) 012.

[47] A. Martin, W. Stirling, R. Thorne, and G. Watt, Eur. Phys. J. C 63, 189 (2009).

[48] R. D. Ball, V. Bertone, F. Cerutti, L. Del Debbio, S. Forte et al., Nucl. Phys. B849, 296 (2011).

[49] P.Z. Skands, Phys. Rev. D 82, 074018 (2010).

[50] M. Bahr, S. Gieseke, M. Gigg, D. Grellscheid, K. Hamilton et al., Eur. Phys. J. C 58, 639 (2008).

[51] CMS Collaboration, Phys. Rev. D 84, 052011 (2011).

G. Aad ${ }^{47}$ B. Abbott,${ }^{109}$ J. Abdallah, ${ }^{11}$ A. A. Abdelalim, ${ }^{48}$ A. Abdesselam, ${ }^{116}$ O. Abdinov, ${ }^{10}$ B. Abi, ${ }^{110}$ M. Abolins, ${ }^{86}$ O. S. AbouZeid, ${ }^{156}$ H. Abramowicz, ${ }^{151}$ H. Abreu, ${ }^{113}$ E. Acerbi, ${ }^{87 a, 87 b}$ B. S. Acharya, ${ }^{162 a, 162 b}$ L. Adamczyk, ${ }^{37}$ D. L. Adams, ${ }^{24}$ T. N. Addy, ${ }^{55}$ J. Adelman, ${ }^{173}$ M. Aderholz,${ }^{97}$ S. Adomeit,${ }^{96}$ P. Adragna, ${ }^{73}$ T. Adye, ${ }^{127}$ S. Aefsky, ${ }^{22}$ J. A. Aguilar-Saavedra, ${ }^{122 b, a}$ M. Aharrouche, ${ }^{79}$ S. P. Ahlen, ${ }^{21}$ F. Ahles, ${ }^{47}$ A. Ahmad, ${ }^{146}$ M. Ahsan,${ }^{40}$ G. Aielli,,${ }^{131 a, 131 b}$ T. Akdogan, ${ }^{18 a}$ T. P. A. Åkesson, ${ }^{77}$ G. Akimoto, ${ }^{153}$ A. V. Akimov, ${ }^{92}$ A. Akiyama, ${ }^{65}$ M. S. Alam, ${ }^{1}$ M. A. Alam, ${ }^{74}$ J. Albert, ${ }^{167}$ S. Albrand,${ }^{54}$ M. Aleksa, ${ }^{29}$ I. N. Aleksandrov ${ }^{63}$ F. Alessandria,${ }^{87 a}$ C. Alexa, ${ }^{25 a}$ G. Alexander,${ }^{151}$ G. Alexandre, ${ }^{48}$ T. Alexopoulos, ${ }^{9}$ M. Alhroob,${ }^{20}$ M. Aliev, ${ }^{15}$ G. Alimonti,${ }^{87 a}$ J. Alison, ${ }^{118}$ M. Aliyev, ${ }^{10}$ B. M. M. Allbrooke, ${ }^{17}$ P. P. Allport, ${ }^{71}$ S. E. Allwood-Spiers, ${ }^{52}$ J. Almond ${ }^{80}$ A. Aloisio, ${ }^{100 a, 100 b}$ R. Alon, ${ }^{169}$ A. Alonso, ${ }^{77}$ B. Alvarez Gonzalez, ${ }^{86}$ M. G. Alviggi, ${ }^{100 a, 100 b}$ K. Amako, ${ }^{64}$ P. Amaral, ${ }^{29}$ C. Amelung, ${ }^{22}$ V. V. Ammosov, ${ }^{126}$ A. Amorim, ${ }^{122 a, b}$ G. Amorós, ${ }^{165}$ N. Amram,,${ }^{151}$ C. Anastopoulos, ${ }^{29}$ L. S. Ancu, ${ }^{16}$ N. Andari, ${ }^{113}$ T. Andeen, ${ }^{34}$ C. F. Anders,${ }^{20}$ G. Anders,${ }^{57 a}$ K. J. Anderson, ${ }^{30}$ A. Andreazza,${ }^{87 a, 87 b}$ V. Andrei,${ }^{57 a}$ M-L. Andrieux,${ }^{54}$ X. S. Anduaga, ${ }^{68}$ A. Angerami, ${ }^{34}$ F. Anghinolfi, ${ }^{29}$ A. Anisenkov, ${ }^{105}$ N. Anjos, ${ }^{122 a}$ A. Annovi, ${ }^{46}$ A. Antonaki, ${ }^{8}$ M. Antonelli ${ }^{46}$ A. Antonov, ${ }^{94}$ J. Antos, ${ }^{142 b}$ F. Anulli, ${ }^{130 a}$ S. Aoun, ${ }^{81}$ L. Aperio Bella, ${ }^{4}$ R. Apolle, ${ }^{116, c}$ G. Arabidze, ${ }^{86}$ I. Aracena, ${ }^{141}$ Y. Arai, ${ }^{64}$ A. T. H. Arce, ${ }^{44}$ S. Arfaoui, ${ }^{146}$ J-F. Arguin, ${ }^{14}$ E. Arik, ${ }^{18 a, j j}$ M. Arik, ${ }^{18 a}$ A. J. Armbruster ${ }^{85}$ 
O. Arnaez,${ }^{79}$ C. Arnault, ${ }^{113}$ A. Artamonov, ${ }^{93}$ G. Artoni, ${ }^{130 a, 130 b}$ D. Arutinov, ${ }^{20}$ S. Asai, ${ }^{153}$ R. Asfandiyarov, ${ }^{170}$ S. Ask ${ }^{27}$ B. Asman, ${ }^{144 a, 144 b}$ L. Asquith, ${ }^{5}$ K. Assamagan, ${ }^{24}$ A. Astbury, ${ }^{167}$ A. Astvatsatourov, ${ }^{51}$ B. Aubert, ${ }^{4}$ E. Auge, ${ }^{113}$ K. Augsten, ${ }^{125}$ M. Aurousseau, ${ }^{143 a}$ G. Avolio, ${ }^{161}$ R. Avramidou, ${ }^{9}$ D. Axen, ${ }^{166}$ C. Ay,${ }^{53}$ G. Azuelos, ${ }^{91, d}$ Y. Azuma, ${ }^{153}$ M. A. Baak,${ }^{29}$ G. Baccaglioni, ${ }^{87 a}$ C. Bacci, ${ }^{132 a, 132 b}$ A. M. Bach,${ }^{14}$ H. Bachacou, ${ }^{134}$ K. Bachas, ${ }^{29}$ M. Backes, ${ }^{48}$ M. Backhaus, ${ }^{20}$ E. Badescu, ${ }^{25 a}$ P. Bagnaia, ${ }^{130 a, 130 b}$ S. Bahinipati, ${ }^{2}$ Y. Bai, ${ }^{32 a}$ D. C. Bailey ${ }^{156}$ T. Bain, ${ }^{156}$ J. T. Baines, ${ }^{127}$ O. K. Baker, ${ }^{173}$ M. D. Baker, ${ }^{24}$ S. Baker,${ }^{75}$ E. Banas, ${ }^{38}$ P. Banerjee, ${ }^{91}$ Sw. Banerjee, ${ }^{170}$ D. Banfi, ${ }^{29}$ A. Bangert, ${ }^{148}$ V. Bansal, ${ }^{167}$ H. S. Bansil,,${ }^{17}$ L. Barak, ${ }^{169}$ S. P. Baranov, ${ }^{92}$ A. Barashkou, ${ }^{63}$ A. Barbaro Galtieri, ${ }^{14}$ T. Barber, ${ }^{47}$ E. L. Barberio, ${ }^{84}$ D. Barberis, ${ }^{49 a, 49 b}$ M. Barbero, ${ }^{20}$ D. Y. Bardin,${ }^{63}$ T. Barillari, ${ }^{97}$ M. Barisonzi,${ }^{172}$ T. Barklow, ${ }^{141}$ N. Barlow, ${ }^{27}$ B. M. Barnett, ${ }^{127}$ R. M. Barnett, ${ }^{14}$ A. Baroncelli, ${ }^{132 a}$ G. Barone, ${ }^{48}$ A. J. Barr, ${ }^{116}$ F. Barreiro ${ }^{78}$ J. Barreiro Guimarães da Costa, ${ }^{56}$ P. Barrillon, ${ }^{113}$ R. Bartoldus, ${ }^{141}$ A. E. Barton, ${ }^{69}$ V. Bartsch, ${ }^{147}$ R. L. Bates, ${ }^{52}$ L. Batkova, ${ }^{142 a}$ J. R. Batley, ${ }^{27}$ A. Battaglia, ${ }^{16}$ M. Battistin, ${ }^{29}$ F. Bauer, ${ }^{134}$ H. S. Bawa, ${ }^{141, e}$ S. Beale, ${ }^{96}$

T. Beau, ${ }^{76}$ P. H. Beauchemin, ${ }^{159}$ R. Beccherle,${ }^{49 a}$ P. Bechtle, ${ }^{20}$ H. P. Beck,,${ }^{16}$ S. Becker, ${ }^{96}$ M. Beckingham,${ }^{136}$ K. H. Becks, ${ }^{172}$ A. J. Beddall, ${ }^{18 c}$ A. Beddall, ${ }^{18 c}$ S. Bedikian, ${ }^{173}$ V. A. Bednyakov, ${ }^{63}$ C. P. Bee, ${ }^{81}$ M. Begel,${ }^{24}$ S. Behar Harpaz, ${ }^{150}$ P. K. Behera,${ }^{61}$ M. Beimforde, ${ }^{97}$ C. Belanger-Champagne, ${ }^{83}$ P. J. Bell, ${ }^{48}$ W. H. Bell, ${ }^{48}$ G. Bella, ${ }^{151}$ L. Bellagamba, ${ }^{19 a}$ F. Bellina,${ }^{29}$ M. Bellomo, ${ }^{29}$ A. Belloni, ${ }^{56}$ O. Beloborodova, ${ }^{105, f} \mathrm{~K}$. Belotskiy,${ }^{94}$ O. Beltramello, ${ }^{29}$ S. Ben Ami, ${ }^{150}$ O. Benary, ${ }^{151}$ D. Benchekroun, ${ }^{133 a}$ C. Benchouk, ${ }^{81}$ M. Bendel, ${ }^{79}$ N. Benekos, ${ }^{163}$ Y. Benhammou, ${ }^{151}$ E. Benhar Noccioli, ${ }^{48}$ J. A. Benitez Garcia, ${ }^{157 b}$ D. P. Benjamin, ${ }^{44}$ M. Benoit, ${ }^{113}$ J. R. Bensinger, ${ }^{22}$ K. Benslama, ${ }^{128}$ S. Bentvelsen, ${ }^{103}$ D. Berge, ${ }^{29}$ E. Bergeaas Kuutmann,${ }^{41}$ N. Berger ${ }^{4}$ F. Berghaus, ${ }^{167}$ E. Berglund,${ }^{103}$ J. Beringer, ${ }^{14}$ P. Bernat, ${ }^{75}$ R. Bernhard,${ }^{47}$ C. Bernius,${ }^{24}$ T. Berry, ${ }^{74}$ C. Bertella, ${ }^{81}$ A. Bertin, ${ }^{19 a, 19 b}$ F. Bertinelli, ${ }^{29}$ F. Bertolucci, ${ }^{120 \mathrm{a}, 120 \mathrm{~b}}$ M. I. Besana,${ }^{87 \mathrm{a}, 87 \mathrm{~b}}$ N. Besson, ${ }^{134}$ S. Bethke,${ }^{97}$ W. Bhimji, ${ }^{45}$ R. M. Bianchi, ${ }^{29}$ M. Bianco,${ }^{70 a, 70 b}$

O. Biebel, ${ }^{96}$ S. P. Bieniek,${ }^{75}$ K. Bierwagen, ${ }^{53}$ J. Biesiada, ${ }^{14}$ M. Biglietti, ${ }^{132 a}$ H. Bilokon ${ }^{46}$ M. Bindi, ${ }^{19 a, 19 b}$ S. Binet ${ }^{113}$ A. Bingul, ${ }^{18 \mathrm{c}}$ C. Bini,${ }^{130 a, 130 \mathrm{~b}}$ C. Biscarat, ${ }^{175}$ U. Bitenc,${ }^{47}$ K. M. Black, ${ }^{21}$ R. E. Blair, ${ }^{5}$ J.-B. Blanchard, ${ }^{134}$ G. Blanchot, ${ }^{29}$ T. Blazek, ${ }^{142 a}$ C. Blocker, ${ }^{22}$ J. Blocki ${ }^{38}$ A. Blondel,${ }^{48}$ W. Blum,${ }^{79}$ U. Blumenschein, ${ }^{53}$ G. J. Bobbink, ${ }^{103}$ V. B. Bobrovnikov, ${ }^{105}$ S. S. Bocchetta, ${ }^{77}$ A. Bocci, ${ }^{44}$ C. R. Boddy, ${ }^{116}$ M. Boehler, ${ }^{41}$ J. Boek, ${ }^{172}$ N. Boelaert, ${ }^{35}$ J. A. Bogaerts, ${ }^{29}$ A. Bogdanchikov, ${ }^{105}$ A. Bogouch,,${ }^{88, j j}$ C. Bohm, ${ }^{144 a}$ V. Boisvert, ${ }^{74}$ T. Bold, ${ }^{37}$ V. Boldea, ${ }^{25 a}$ N. M. Bolnet, ${ }^{134}$ M. Bona, ${ }^{73}$ V. G. Bondarenko, ${ }^{94}$ M. Bondioli, ${ }^{161}$ M. Boonekamp, ${ }^{134}$ C. N. Booth,${ }^{137}$ S. Bordoni ${ }^{76}$ C. Borer, ${ }^{16}$ A. Borisov, ${ }^{126}$ G. Borissov, ${ }^{69}$ I. Borjanovic, ${ }^{12 a}$ M. Borri, ${ }^{80}$ S. Borroni, ${ }^{85}$

V. Bortolotto, ${ }^{132 \mathrm{a}, 132 \mathrm{~b}} \mathrm{~K}$. Bos, ${ }^{103}$ D. Boscherini, ${ }^{19 \mathrm{a}} \mathrm{M}$. Bosman, ${ }^{11}$ H. Boterenbrood, ${ }^{103}$ D. Botterill, ${ }^{127} \mathrm{~J}$. Bouchami, ${ }^{91}$ J. Boudreau, ${ }^{121}$ E. V. Bouhova-Thacker, ${ }^{69}$ D. Boumediene, ${ }^{33}$ C. Bourdarios,${ }^{113}$ N. Bousson, ${ }^{81}$ A. Boveia,${ }^{30}$ J. Boyd, ${ }^{29}$ I. R. Boyko, ${ }^{63}$ N. I. Bozhko, ${ }^{126}$ I. Bozovic-Jelisavcic, ${ }^{12 b}$ J. Bracinik, ${ }^{17}$ A. Braem, ${ }^{29}$ P. Branchini, ${ }^{132 a}$ G. W. Brandenburg, ${ }^{56}$ A. Brandt, ${ }^{7}$ G. Brandt,,${ }^{116}$ O. Brandt,${ }^{53}$ U. Bratzler, ${ }^{154}$ B. Brau, ${ }^{82}$ J. E. Brau, ${ }^{112}$ H. M. Braun, ${ }^{172}$ B. Brelier, ${ }^{156}$ J. Bremer, ${ }^{29}$ R. Brenner, ${ }^{164}$ S. Bressler, ${ }^{169}$ D. Britton, ${ }^{52}$ F. M. Brochu, ${ }^{27}$ I. Brock, ${ }^{20}$ R. Brock,${ }^{86}$ T. J. Brodbeck, ${ }^{69}$ E. Brodet, ${ }^{151}$ F. Broggi, ${ }^{87 a}$ C. Bromberg, ${ }^{86}$ J. Bronner, ${ }^{97}$ G. Brooijmans, ${ }^{34}$ W. K. Brooks, ${ }^{31 b}$ G. Brown, ${ }^{80}$ H. Brown, ${ }^{7}$ P. A. Bruckman de Renstrom, ${ }^{38}$ D. Bruncko, ${ }^{142 b}$ R. Bruneliere, ${ }^{47}$ S. Brunet, ${ }^{59}$ A. Bruni, ${ }^{19 a}$ G. Bruni, ${ }^{19 a}$ M. Bruschi, ${ }^{19 a}$ T. Buanes, ${ }^{13}$ Q. Buat,${ }^{54}$ F. Bucci, ${ }^{48}$ J. Buchanan, ${ }^{116}$ N. J. Buchanan, ${ }^{2}$ P. Buchholz, ${ }^{139}$ R. M. Buckingham, ${ }^{116}$ A. G. Buckley ${ }^{45}$ S. I. Buda, ${ }^{25 a}$ I. A. Budagov, ${ }^{63}$ B. Budick,${ }^{106}$ V. Büscher,${ }^{79}$ L. Bugge,${ }^{115}$ O. Bulekov, ${ }^{94}$ M. Bunse, ${ }^{42}$ T. Buran, ${ }^{115}$ H. Burckhart ${ }^{29}$ S. Burdin, ${ }^{71}$ T. Burgess, ${ }^{13}$ S. Burke, ${ }^{127}$ E. Busato,${ }^{33}$

P. Bussey, ${ }^{52}$ C. P. Buszello, ${ }^{164}$ F. Butin, ${ }^{29}$ B. Butler, ${ }^{141}$ J. M. Butler, ${ }^{21}$ C. M. Buttar, ${ }^{52}$ J. M. Butterworth, ${ }^{75}$ W. Buttinger, ${ }^{27}$ S. Cabrera Urbán, ${ }^{165}$ D. Caforio, ${ }^{19 a, 19 b}$ O. Cakir,${ }^{3 a}$ P. Calafiura, ${ }^{14}$ G. Calderini, ${ }^{76}$ P. Calfayan, ${ }^{96}$ R. Calkins, ${ }^{104}$ L. P. Caloba, ${ }^{23 a}$ R. Caloi,${ }^{130 a, 130 b}$ D. Calvet,${ }^{33}$ S. Calvet, ${ }^{33}$ R. Camacho Toro, ${ }^{33}$ P. Camarri, ${ }^{131 a, 131 b}$ M. Cambiaghi, ${ }^{17 \mathrm{a}, 117 \mathrm{~b}}$ D. Cameron, ${ }^{115}$ L. M. Caminada, ${ }^{14}$ S. Campana,${ }^{29}$ M. Campanelli, ${ }^{75}$ V. Canale,${ }^{100 a, 100 b}$ F. Canelli, ${ }^{30, g}$ A. Canepa,${ }^{157 a}$ J. Cantero,${ }^{78}$ L. Capasso, ${ }^{100 a, 100 b}$ M. D. M. Capeans Garrido, ${ }^{29}$ I. Caprini, ${ }^{25 a}$ M. Caprini, ${ }^{25 a}$ D. Capriotti, ${ }^{97}$ M. Capua ${ }^{36 a, 36 b}$ R. Caputo, ${ }^{79}$ C. Caramarcu ${ }^{24}$ R. Cardarelli, ${ }^{131 a}$ T. Carli, ${ }^{29}$ G. Carlino, ${ }^{100 a}$ L. Carminati, ${ }^{87 a, 87 b}$ B. Caron,${ }^{83}$ S. Caron, ${ }^{102}$ G. D. Carrillo Montoya, ${ }^{170}$ A. A. Carter, ${ }^{73}$ J. R. Carter, ${ }^{27}$ J. Carvalho, ${ }^{122 a, h}$ D. Casadei, ${ }^{106}$ M. P. Casado, ${ }^{11}$ M. Cascella, ${ }^{120 a, 120 b}$ C. Caso, ${ }^{49 a, 49 b, j j}$

A. M. Castaneda Hernandez, ${ }^{170}$ E. Castaneda-Miranda, ${ }^{170}$ V. Castillo Gimenez, ${ }^{165}$ N. F. Castro, ${ }^{122 a}$ G. Cataldi, ${ }^{70 a}$ F. Cataneo, ${ }^{29}$ A. Catinaccio, ${ }^{29}$ J. R. Catmore, ${ }^{29}$ A. Cattai, ${ }^{29}$ G. Cattani, ${ }^{131 \mathrm{a}, 131 \mathrm{~b}}$ S. Caughron, ${ }^{86}$ D. Cauz, ${ }^{162 \mathrm{a}, 162 \mathrm{c}}$ P. Cavalleri, ${ }^{76}$ D. Cavalli, ${ }^{87 a}$ M. Cavalli-Sforza, ${ }^{11}$ V. Cavasinni, ${ }^{120 a, 120 b}$ F. Ceradini, ${ }^{132 a, 132 b}$ A. S. Cerqueira, ${ }^{23 b}$ A. Cerri, ${ }^{29}$ L. Cerrito, ${ }^{73}$ F. Cerutti, ${ }^{46}$ S. A. Cetin, ${ }^{18 b}$ F. Cevenini, ${ }^{100 a, 100 b}$ A. Chafaq, ${ }^{133 a}$ D. Chakraborty ${ }^{104}$ K. Chan, ${ }^{2}$ B. Chapleau, ${ }^{83}$ J. D. Chapman, ${ }^{27}$ J. W. Chapman, ${ }^{85}$ E. Chareyre, ${ }^{76}$ D. G. Charlton, ${ }^{17}$ V. Chavda,${ }^{80}$

C. A. Chavez Barajas, ${ }^{29}$ S. Cheatham, ${ }^{83}$ S. Chekanov, ${ }^{5}$ S. V. Chekulaev, ${ }^{157 a}$ G. A. Chelkov,${ }^{63}$ M. A. Chelstowska, ${ }^{102}$ 
C. Chen, ${ }^{62}$ H. Chen, ${ }^{24}$ S. Chen, ${ }^{32 \mathrm{c}}$ T. Chen,${ }^{32 \mathrm{c}}$ X. Chen, ${ }^{170}$ S. Cheng, ${ }^{32 \mathrm{a}}$ A. Cheplakov ${ }^{63}$ V. F. Chepurnov, ${ }^{63}$ R. Cherkaoui El Moursli, ${ }^{133 e}$ V. Chernyatin,${ }^{24}$ E. Cheu, ${ }^{6}$ S. L. Cheung, ${ }^{156}$ L. Chevalier, ${ }^{134}$ G. Chiefari, ${ }^{100 a, 100 b}$ L. Chikovani, ${ }^{50 a}$ J. T. Childers ${ }^{29}$ A. Chilingarov ${ }^{69}$ G. Chiodini ${ }^{70 a}$ A. S. Chisholm,${ }^{17}$ M. V. Chizhov, ${ }^{63}$ G. Choudalakis, ${ }^{30}$ S. Chouridou, ${ }^{135}$ I. A. Christidi, ${ }^{75}$ A. Christov, ${ }^{47}$ D. Chromek-Burckhart, ${ }^{29}$ M. L. Chu, ${ }^{149}$ J. Chudoba, ${ }^{123}$ G. Ciapetti, ${ }^{130 a, 130 b}$ K. Ciba, ${ }^{37}$ A. K. Ciftci, ${ }^{3 a}$ R. Ciftci, ${ }^{3 a}$ D. Cinca, ${ }^{33}$ V. Cindro, ${ }^{72}$ M. D. Ciobotaru, ${ }^{161}$ C. Ciocca, ${ }^{19 a}$ A. Ciocio, ${ }^{14}$ M. Cirilli,${ }^{85}$ M. Citterio, ${ }^{87 a}$ M. Ciubancan, ${ }^{25 a}$ A. Clark,${ }^{48}$ P. J. Clark,${ }^{45}$ W. Cleland,${ }^{121}$ J. C. Clemens, ${ }^{81}$ B. Clement, ${ }^{54}$ C. Clement, ${ }^{144 a, 144 b}$ R. W. Clifft, ${ }^{127}$ Y. Coadou, ${ }^{81}$ M. Cobal, ${ }^{162 a, 162 c}$ A. Coccaro, ${ }^{170}$ J. Cochran, ${ }^{62}$ P. Coe, ${ }^{116}$ J. G. Cogan, ${ }^{141}$ J. Coggeshall, ${ }^{163}$ E. Cogneras, ${ }^{175}$ J. Colas, ${ }^{4}$ A. P. Colijn, ${ }^{103}$ N. J. Collins, ${ }^{17}$ C. Collins-Tooth, ${ }^{52}$ J. Collot, ${ }^{54}$ G. Colon, ${ }^{82}$ P. Conde Muiño, ${ }^{122 a}$ E. Coniavitis, ${ }^{116}$ M. C. Conidi, ${ }^{11}$ M. Consonni, ${ }^{102}$ V. Consorti, ${ }^{47}$ S. Constantinescu, ${ }^{25 a}$ C. Conta, ${ }^{117 a, 117 b}$ F. Conventi, ${ }^{100 a, i}$ J. Cook,${ }^{29}$ M. Cooke, ${ }^{14}$ B. D. Cooper, ${ }^{75}$ A. M. Cooper-Sarkar, ${ }^{116}$ K. Copic, ${ }^{14}$ T. Cornelissen, ${ }^{172}$ M. Corradi, ${ }^{19 a}$ F. Corriveau, ${ }^{83, j}$ A. Cortes-Gonzalez, ${ }^{163}$ G. Cortiana, ${ }^{97}$ G. Costa, ${ }^{87 a}$ M. J. Costa, ${ }^{165}$ D. Costanzo, ${ }^{137}$ T. Costin, ${ }^{30}$ D. Côté, ${ }^{29}$ R. Coura Torres,${ }^{23 a}$ L. Courneyea, ${ }^{167}$ G. Cowan, ${ }^{74}$ C. Cowden, ${ }^{27}$ B. E. Cox ${ }^{80}$ K. Cranmer, ${ }^{106}$ F. Crescioli, ${ }^{120 a, 120 b}$ M. Cristinziani, ${ }^{20}$ G. Crosetti, ${ }^{36 a, 36 b}$ R. Crupi,${ }^{70 a, 70 b}$ S. Crépé-Renaudin, ${ }^{54}$ C.-M. Cuciuc ${ }^{25 a}$ C. Cuenca Almenar, ${ }^{173}$

T. Cuhadar Donszelmann, ${ }^{137}$ M. Curatolo, ${ }^{46}$ C. J. Curtis, ${ }^{17}$ C. Cuthbert, ${ }^{148}$ P. Cwetanski, ${ }^{59}$ H. Czirr, ${ }^{139}$ P. Czodrowski, ${ }^{43}$ Z. Czyczula, ${ }^{173}$ S. D'Auria, ${ }^{52}$ M. D'Onofrio, ${ }^{71}$ A. D'Orazio, ${ }^{130 a, 130 b}$ P. V. M. Da Silva, ${ }^{23 a}$

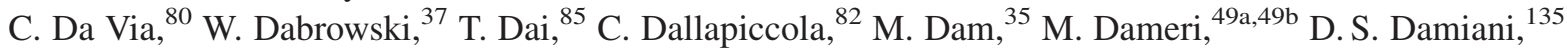
H. O. Danielsson, ${ }^{29}$ D. Dannheim, ${ }^{97}$ V. Dao, ${ }^{48}$ G. Darbo, ${ }^{49 a}$ G. L. Darlea, ${ }^{25 b}$ W. Davey, ${ }^{20}$ T. Davidek, ${ }^{124}$ N. Davidson,${ }^{84}$ R. Davidson, ${ }^{69}$ E. Davies, ${ }^{116, c}$ M. Davies, ${ }^{91}$ A. R. Davison, ${ }^{75}$ Y. Davygora, ${ }^{57 a}$ E. Dawe,${ }^{140}$ I. Dawson, ${ }^{137}$ J. W. Dawson, ${ }^{5, j j}$ R. K. Daya-Ishmukhametova, ${ }^{22}$ K. De,${ }^{7}$ R. de Asmundis, ${ }^{100 a}$ S. De Castro, ${ }^{19 a, 19 b}$ P. E. De Castro Faria Salgado, ${ }^{24}$ S. De Cecco, ${ }^{76}$ J. de Graat, ${ }^{96}$ N. De Groot, ${ }^{102}$ P. de Jong, ${ }^{103}$ C. De La Taille, ${ }^{113}$

H. De la Torre ${ }^{78}$ B. De Lotto, ${ }^{162 a, 162 c}$ L. de Mora, ${ }^{69}$ L. De Nooij,${ }^{103}$ D. De Pedis, ${ }^{130 a}$ A. De Salvo, ${ }^{130 a}$ U. De Sanctis, ${ }^{162 a, 162 c}$ A. De Santo, ${ }^{147}$ J. B. De Vivie De Regie, ${ }^{113}$ S. Dean, ${ }^{75}$ W. J. Dearnaley, ${ }^{69}$ R. Debbe,${ }^{24}$

C. Debenedetti ${ }^{45}$ D. V. Dedovich, ${ }^{63}$ J. Degenhardt,${ }^{118}$ M. Dehchar, ${ }^{116}$ C. Del Papa, ${ }^{162 a, 162 \mathrm{c}}$ J. Del Peso, ${ }^{78}$ T. Del Prete, ${ }^{120 a, 120 b}$ T. Delemontex,${ }^{54}$ M. Deliyergiyev, ${ }^{72}$ A. Dell'Acqua, ${ }^{29}$ L. Dell'Asta, ${ }^{21}$ M. Della Pietra, ${ }^{100 a, i}$ D. della Volpe, ${ }^{100 a, 100 b}$ M. Delmastro, ${ }^{4}$ N. Delruelle, ${ }^{29}$ P. A. Delsart, ${ }^{54}$ C. Deluca, ${ }^{146}$ S. Demers,${ }^{173}$ M. Demichev, ${ }^{63}$ B. Demirkoz, ${ }^{11, k}$ J. Deng, ${ }^{161}$ S. P. Denisov ${ }^{126}$ D. Derendarz,${ }^{38}$ J. E. Derkaoui, ${ }^{133 d}$ F. Derue,${ }^{76}$ P. Dervan,${ }^{71}$ K. Desch, ${ }^{20}$ E. Devetak, ${ }^{146}$ P. O. Deviveiros, ${ }^{103}$ A. Dewhurst,${ }^{127}$ B. DeWilde, ${ }^{146}$ S. Dhaliwal, ${ }^{156}$ R. Dhullipudi, ${ }^{24,1}$ A. Di Ciaccio, ${ }^{131 \mathrm{a}, 131 \mathrm{~b}}$ L. Di Ciaccio, ${ }^{4}$ A. Di Girolamo, ${ }^{29}$ B. Di Girolamo, ${ }^{29}$ S. Di Luise, ${ }^{132 a, 132 b}$ A. Di Mattia, ${ }^{170}$ B. Di Micco ${ }^{29}$ R. Di Nardo, ${ }^{46}$ A. Di Simone,${ }^{131 a, 131 b}$ R. Di Sipio, ${ }^{19 a, 19 b}$ M. A. Diaz, ${ }^{31 a}$ F. Diblen, ${ }^{18 c}$ E. B. Diehl,${ }^{85}$ J. Dietrich ${ }^{41}$ T. A. Dietzsch, ${ }^{57 a}$ S. Diglio, ${ }^{84}$ K. Dindar Yagci, ${ }^{39}$ J. Dingfelder, ${ }^{20}$ C. Dionisi, ${ }^{130 a, 130 b}$ P. Dita, ${ }^{25 a}$ S. Dita, ${ }^{25 a}$ F. Dittus,${ }^{29}$ F. Djama, ${ }^{81}$ T. Djobava, ${ }^{50 b}$ M. A. B. do Vale, ${ }^{23 c}$ A. Do Valle Wemans, ${ }^{122 a}$ T. K. O. Doan,${ }^{4}$ M. Dobbs ${ }^{83}$ R. Dobinson, ${ }^{29, j j}$ D. Dobos, ${ }^{29}$ E. Dobson,${ }^{29, m}$ J. Dodd,${ }^{34}$ C. Doglioni,${ }^{48}$ T. Doherty, ${ }^{52}$ Y. Doi,${ }^{64, j j}$ J. Dolejsi, ${ }^{124}$ I. Dolenc, ${ }^{72}$ Z. Dolezal, ${ }^{124}$ B. A. Dolgoshein, ${ }^{94, j j}$ T. Dohmae, ${ }^{153}$ M. Donadelli, ${ }^{23 d}$ M. Donega, ${ }^{118}$ J. Donini, ${ }^{33}$ J. Dopke, ${ }^{29}$ A. Doria, ${ }^{100 a}$ A. Dos Anjos, ${ }^{170}$ M. Dosil, ${ }^{11}$ A. Dotti, ${ }^{120 a, 120 b}$ M. T. Dova, ${ }^{68}$ J. D. Dowell, ${ }^{17}$ A. D. Doxiadis, ${ }^{103}$ A. T. Doyle, ${ }^{52}$ Z. Drasal, ${ }^{124}$ J. Drees, ${ }^{172}$ N. Dressnandt, ${ }^{118}$ H. Drevermann, ${ }^{29}$ C. Driouichi, ${ }^{35}$ M. Dris, ${ }^{9}$ J. Dubbert, ${ }^{97}$ S. Dube, ${ }^{14}$ E. Duchovni, ${ }^{169}$ G. Duckeck, ${ }^{96}$ A. Dudarev, ${ }^{29}$ F. Dudziak,${ }^{62}$ M. Dührssen,${ }^{29}$ I. P. Duerdoth ${ }^{80}$ L. Duflot, ${ }^{113}$ M-A. Dufour, ${ }^{83}$ M. Dunford ${ }^{29}$ H. Duran Yildiz, ${ }^{3 a}$ R. Duxfield, ${ }^{137}$ M. Dwuznik, ${ }^{37}$

F. Dydak,${ }^{29}$ M. Düren, ${ }^{51}$ W. L. Ebenstein, ${ }^{44}$ J. Ebke, ${ }^{96}$ S. Eckweiler,${ }^{79}$ K. Edmonds, ${ }^{79}$ C. A. Edwards, ${ }^{74}$ N. C. Edwards, ${ }^{52}$ W. Ehrenfeld, ${ }^{41}$ T. Ehrich, ${ }^{97}$ T. Eifert, ${ }^{141}$ G. Eigen, ${ }^{13}$ K. Einsweiler, ${ }^{14}$ E. Eisenhandler, ${ }^{73}$ T. Ekelof, ${ }^{164}$ M. El Kacimi, ${ }^{133 c}$ M. Ellert, ${ }^{164}$ S. Elles, ${ }^{4}$ F. Ellinghaus, ${ }^{79}$ K. Ellis, ${ }^{73}$ N. Ellis, ${ }^{29}$ J. Elmsheuser, ${ }^{96}$ M. Elsing, ${ }^{29}$ D. Emeliyanov, ${ }^{127}$ R. Engelmann, ${ }^{146}$ A. Engl, ${ }^{96}$ B. Epp, ${ }^{60}$ A. Eppig, ${ }^{85}$ J. Erdmann, ${ }^{53}$ A. Ereditato, ${ }^{16}$ D. Eriksson, ${ }^{144 a}$ J. Ernst, ${ }^{1}$ M. Ernst, ${ }^{24}$ J. Ernwein, ${ }^{134}$ D. Errede, ${ }^{163}$ S. Errede,${ }^{163}$ E. Ertel,${ }^{79}$ M. Escalier,${ }^{113}$

C. Escobar, ${ }^{121}$ X. Espinal Curull, ${ }^{11}$ B. Esposito, ${ }^{46}$ F. Etienne, ${ }^{81}$ A. I. Etienvre, ${ }^{134}$ E. Etzion, ${ }^{151}$ D. Evangelakou, ${ }^{53}$ H. Evans, ${ }^{59}$ L. Fabbri, ${ }^{19 a, 19 b}$ C. Fabre,${ }^{29}$ R. M. Fakhrutdinov,${ }^{126}$ S. Falciano, ${ }^{130 a}$ Y. Fang, ${ }^{170}$ M. Fanti,,${ }^{87,87 b}$ A. Farbin, ${ }^{7}$ A. Farilla, ${ }^{132 a}$ J. Farley, ${ }^{146}$ T. Farooque, ${ }^{156}$ S. M. Farrington, ${ }^{116}$ P. Farthouat, ${ }^{29}$ P. Fassnacht,${ }^{29}$ D. Fassouliotis, ${ }^{8}$ B. Fatholahzadeh, ${ }^{156}$ A. Favareto, ${ }^{87 a, 87 b}$ L. Fayard, ${ }^{113}$ S. Fazio, ${ }^{36 a, 36 b}$ R. Febbraro, ${ }^{33}$ P. Federic, ${ }^{142 a}$ O. L. Fedin, ${ }^{119}$ W. Fedorko,${ }^{86}$ M. Fehling-Kaschek, ${ }^{47}$ L. Feligioni, ${ }^{81}$ D. Fellmann, ${ }^{5}$ C. Feng, ${ }^{32 d}$ E. J. Feng, ${ }^{30}$ A. B. Fenyuk, ${ }^{126}$ J. Ferencei, ${ }^{142 \mathrm{~b}}$ J. Ferland,${ }^{91}$ W. Fernando, ${ }^{107}$ S. Ferrag,${ }^{52}$ J. Ferrando,${ }^{52}$ V. Ferrara, ${ }^{41}$ A. Ferrari, ${ }^{164}$ P. Ferrari, ${ }^{103}$ R. Ferrari, ${ }^{117}$ D. E. Ferreira de Lima, ${ }^{52}$ A. Ferrer, ${ }^{165}$ M. L. Ferrer, ${ }^{46}$ D. Ferrere, ${ }^{48}$ C. Ferretti, ${ }^{85}$ A. Ferretto Parodi, ${ }^{49 a, 49 b}$ M. Fiascaris,${ }^{30}$ F. Fiedler, ${ }^{79}$ A. Filipčič,${ }^{72}$ A. Filippas, ${ }^{9}$ F. Filthaut, ${ }^{102}$ M. Fincke-Keeler, ${ }^{167}$ 
M. C. N. Fiolhais, ${ }^{122 a, h}$ L. Fiorini, ${ }^{165}$ A. Firan, ${ }^{39}$ G. Fischer, ${ }^{41}$ P. Fischer, ${ }^{20}$ M. J. Fisher, ${ }^{107}$ M. Flechl,${ }^{47}$ I. Fleck, ${ }^{139}$ J. Fleckner, ${ }^{79}$ P. Fleischmann, ${ }^{171}$ S. Fleischmann, ${ }^{172}$ T. Flick, ${ }^{172}$ A. Floderus, ${ }^{77}$ L. R. Flores Castillo, ${ }^{170}$ M. J. Flowerdew, ${ }^{97}$ M. Fokitis, ${ }^{9}$ T. Fonseca Martin, ${ }^{16}$ D. A. Forbush, ${ }^{136}$ A. Formica, ${ }^{134}$ A. Forti,${ }^{80}$ D. Fortin, ${ }^{157 a}$ J. M. Foster ${ }^{80}$ D. Fournier, ${ }^{113}$ A. Foussat, ${ }^{29}$ A. J. Fowler, ${ }^{44}$ K. Fowler, ${ }^{135}$ H. Fox,${ }^{69}$ P. Francavilla, ${ }^{11}$ S. Franchino, ${ }^{17 \mathrm{a}, 117 \mathrm{~b}}$ D. Francis, ${ }^{29}$ T. Frank, ${ }^{169}$ M. Franklin, ${ }^{56}$ S. Franz ${ }^{29}$ M. Fraternali, ${ }^{117 a, 117 b}$ S. Fratina, ${ }^{118}$ S. T. French, ${ }^{27}$ F. Friedrich, ${ }^{43}$ R. Froeschl, ${ }^{29}$ D. Froidevaux, ${ }^{29}$ J. A. Frost, ${ }^{27}$ C. Fukunaga, ${ }^{154}$ E. Fullana Torregrosa, ${ }^{29}$ J. Fuster, ${ }^{165}$ C. Gabaldon, ${ }^{29}$ O. Gabizon, ${ }^{169}$ T. Gadfort,${ }^{24}$ S. Gadomski, ${ }^{48}$ G. Gagliardi, ${ }^{49 a, 49 b}$ P. Gagnon,,${ }^{59}$ C. Galea, ${ }^{96}$ E. J. Gallas, ${ }^{116}$ V. Gallo, ${ }^{16}$ B. J. Gallop, ${ }^{127}$ P. Gallus, ${ }^{123}$ K. K. Gan, ${ }^{107}$ Y. S. Gao, ${ }^{141, e}$ V. A. Gapienko, ${ }^{126}$ A. Gaponenko, ${ }^{14}$ F. Garberson, ${ }^{173}$ M. Garcia-Sciveres,${ }^{14}$ C. García, ${ }^{165}$ J. E. García Navarro, ${ }^{165}$ R. W. Gardner, ${ }^{30}$ N. Garelli, ${ }^{29}$ H. Garitaonandia, ${ }^{103}$ V. Garonne, ${ }^{29}$ J. Garvey ${ }^{17}$ C. Gatti, ${ }^{46}$ G. Gaudio, ${ }^{117 a}$ B. Gaur,${ }^{139}$ L. Gauthier, ${ }^{134}$ I. L. Gavrilenko, ${ }^{92}$ C. Gay, ${ }^{166}$ G. Gaycken,${ }^{20}$ J-C. Gayde, ${ }^{29}$ E. N. Gazis, ${ }^{9}$ P. Ge,${ }^{32 d}$ C. N. P. Gee,${ }^{127}$ D. A. A. Geerts, ${ }^{103}$ Ch. Geich-Gimbel,${ }^{20}$ K. Gellerstedt, ${ }^{144 a, 144 b}$ C. Gemme, ${ }^{49 a}$ A. Gemmell, ${ }^{52}$ M. H. Genest, ${ }^{54}$ S. Gentile, ${ }^{130 a, 130 b}$ M. George, ${ }^{53}$ S. George,${ }^{74}$ P. Gerlach, ${ }^{172}$ A. Gershon, ${ }^{151}$ C. Geweniger, ${ }^{57 a}$ H. Ghazlane, ${ }^{133 b}$ N. Ghodbane, ${ }^{33}$ B. Giacobbe, ${ }^{19 \mathrm{a}}$ S. Giagu, ${ }^{130 a, 130 \mathrm{~b}}$ V. Giakoumopoulou ${ }^{8}$ V. Giangiobbe,${ }^{11}$ F. Gianotti, ${ }^{29}$ B. Gibbard, ${ }^{24}$ A. Gibson, ${ }^{156}$ S. M. Gibson, ${ }^{29}$ L. M. Gilbert, ${ }^{116}$ V. Gilewsky, ${ }^{89}$ D. Gillberg, ${ }^{28}$ A. R. Gillman, ${ }^{127}$ D. M. Gingrich, ${ }^{2, d}$ J. Ginzburg, ${ }^{151}$ N. Giokaris, ${ }^{8}$ M. P. Giordani, ${ }^{162 \mathrm{c}}$ R. Giordano, ${ }^{100 a, 100 b}$ F. M. Giorgi, ${ }^{15}$ P. Giovannini, ${ }^{97}$ P. F. Giraud, ${ }^{134}$ D. Giugni, ${ }^{87 a}$ M. Giunta, ${ }^{91}$ P. Giusti, ${ }^{19 a}$ B. K. Gjelsten, ${ }^{115}$ L. K. Gladilin, ${ }^{95}$ C. Glasman, ${ }^{78}$ J. Glatzer, ${ }^{47}$ A. Glazov, ${ }^{41}$ K. W. Glitza, ${ }^{172}$ G. L. Glonti, ${ }^{63}$ J. R. Goddard, ${ }^{73}$ J. Godfrey,${ }^{140}$ J. Godlewski, ${ }^{29}$ M. Goebel, ${ }^{41}$ T. Göpfert, ${ }^{43}$ C. Goeringer, ${ }^{79}$ C. Gössling, ${ }^{42}$ T. Göttfert, ${ }^{97}$ S. Goldfarb,${ }^{85}$ T. Golling,${ }^{173}$ A. Gomes, ${ }^{122 a, b}$ L. S. Gomez Fajardo, ${ }^{41}$ R. Gonçalo, ${ }^{74}$ J. Goncalves Pinto Firmino Da Costa, ${ }^{41}$ L. Gonella, ${ }^{20}$ A. Gonidec, ${ }^{29}$ S. Gonzalez, ${ }^{170}$ S. González de la Hoz, ${ }^{165}$ G. Gonzalez Parra, ${ }^{11}$ M. L. Gonzalez Silva, ${ }^{26}$ S. Gonzalez-Sevilla, ${ }^{48}$ J. J. Goodson, ${ }^{146}$ L. Goossens, ${ }^{29}$ P. A. Gorbounov,${ }^{93}$ H. A. Gordon,${ }^{24}$ I. Gorelov, ${ }^{101}$ G. Gorfine, ${ }^{172}$ B. Gorini, ${ }^{29}$ E. Gorini, ${ }^{70 a, 70 b}$ A. Gorišek, ${ }^{72}$ E. Gornicki, ${ }^{38}$ S. A. Gorokhov, ${ }^{126}$ V. N. Goryachev, ${ }^{126}$ B. Gosdzik, ${ }^{41}$ M. Gosselink, ${ }^{103}$ M. I. Gostkin, ${ }^{63}$ I. Gough Eschrich, ${ }^{161}$ M. Gouighri, ${ }^{133 a}$ D. Goujdami,${ }^{133 c}$ M. P. Goulette, ${ }^{48}$ A. G. Goussiou, ${ }^{136}$ C. Goy, ${ }^{4}$ S. Gozpinar, ${ }^{22}$ I. Grabowska-Bold, ${ }^{37}$ P. Grafström, ${ }^{29}$ K-J. Grahn,,${ }^{41}$ F. Grancagnolo, ${ }^{70 a}$ S. Grancagnolo, ${ }^{15}$ V. Grassi, ${ }^{146}$ V. Gratchev, ${ }^{119}$ N. Grau, ${ }^{34}$ H. M. Gray, ${ }^{29}$ J. A. Gray, ${ }^{146}$ E. Graziani, ${ }^{132 a}$ O. G. Grebenyuk, ${ }^{119}$ T. Greenshaw, ${ }^{71}$ Z. D. Greenwood,${ }^{24,1}$ K. Gregersen, ${ }^{35}$ I. M. Gregor, ${ }^{41}$ P. Grenier, ${ }^{141}$ J. Griffiths, ${ }^{136}$ N. Grigalashvili, ${ }^{63}$ A. A. Grillo, ${ }^{135}$ S. Grinstein, ${ }^{11}$ Y. V. Grishkevich, ${ }^{95}$ J.-F. Grivaz, ${ }^{113}$ M. Groh, ${ }^{97}$ E. Gross, ${ }^{169}$ J. Grosse-Knetter, ${ }^{53}$ J. Groth-Jensen, ${ }^{169}$ K. Grybel, ${ }^{139}$ V. J. Guarino, ${ }^{5}$ D. Guest,${ }^{173}$ C. Guicheney,${ }^{33}$ A. Guida, ${ }^{70 a, 70 b}$ S. Guindon, ${ }^{53}$

H. Guler, ${ }^{83, n}$ J. Gunther, ${ }^{123}$ B. Guo, ${ }^{156}$ J. Guo, ${ }^{34}$ A. Gupta, ${ }^{30}$ Y. Gusakov, ${ }^{63}$ V. N. Gushchin, ${ }^{126}$ P. Gutierrez, ${ }^{109}$ N. Guttman, ${ }^{151}$ O. Gutzwiller, ${ }^{170}$ C. Guyot, ${ }^{134}$ C. Gwenlan, ${ }^{116}$ C. B. Gwilliam, ${ }^{71}$ A. Haas, ${ }^{141}$ S. Haas, ${ }^{29}$ C. Haber,${ }^{14}$ H. K. Hadavand, ${ }^{39}$ D. R. Hadley, ${ }^{17}$ P. Haefner,${ }^{97}$ F. Hahn, ${ }^{29}$ S. Haider, ${ }^{29}$ Z. Hajduk, ${ }^{38}$ H. Hakobyan, ${ }^{174}$ D. Hall, ${ }^{116}$ J. Haller, ${ }^{53}$ K. Hamacher, ${ }^{172}$ P. Hamal, ${ }^{111}$ M. Hamer ${ }^{53}$ A. Hamilton, ${ }^{143 b, o}$ S. Hamilton, ${ }^{159}$ H. Han, ${ }^{32 a}$ L. Han, ${ }^{32 b}$ K. Hanagaki, ${ }^{114}$ K. Hanawa, ${ }^{158}$ M. Hance,,${ }^{14}$ C. Handel, ${ }^{79}$ P. Hanke, ${ }^{57 a}$ J. R. Hansen, ${ }^{35}$ J. B. Hansen,${ }^{35}$ J. D. Hansen, ${ }^{35}$ P. H. Hansen, ${ }^{35}$ P. Hansson, ${ }^{141}$ K. Hara, ${ }^{158}$ G. A. Hare, ${ }^{135}$ T. Harenberg, ${ }^{172}$ S. Harkusha, ${ }^{88}$ D. Harper, ${ }^{85}$ R. D. Harrington, ${ }^{45}$ O. M. Harris, ${ }^{136}$ K. Harrison, ${ }^{17}$ J. Hartert, ${ }^{47}$ F. Hartjes, ${ }^{103}$ T. Haruyama, ${ }^{64}$ A. Harvey, ${ }^{55}$ S. Hasegawa, ${ }^{99}$ Y. Hasegawa, ${ }^{138}$ S. Hassani, ${ }^{134}$ M. Hatch, ${ }^{29}$ D. Hauff, ${ }^{97}$ S. Haug, ${ }^{16}$ M. Hauschild, ${ }^{29}$ R. Hauser, ${ }^{86}$ M. Havranek, ${ }^{20}$ B. M. Hawes, ${ }^{116}$ C. M. Hawkes ${ }^{17}$ R. J. Hawkings,${ }^{29}$ A. D. Hawkins, ${ }^{77}$ D. Hawkins, ${ }^{161}$

T. Hayakawa, ${ }^{65}$ T. Hayashi, ${ }^{158}$ D. Hayden,${ }^{74}$ H. S. Hayward, ${ }^{71}$ S. J. Haywood, ${ }^{127}$ E. Hazen, ${ }^{21}$ M. He ${ }^{32 d}$ S. J. Head, ${ }^{17}$ V. Hedberg, ${ }^{77}$ L. Heelan, ${ }^{7}$ S. Heim, ${ }^{86}$ B. Heinemann, ${ }^{14}$ S. Heisterkamp, ${ }^{35}$ L. Helary, ${ }^{4}$ C. Heller,${ }^{96}$ M. Heller ${ }^{29}$ S. Hellman, ${ }^{144 a, 144 b}$ D. Hellmich,${ }^{20}$ C. Helsens, ${ }^{11}$ R. C. W. Henderson,${ }^{69}$ M. Henke, ${ }^{57 a}$ A. Henrichs,${ }^{53}$

A. M. Henriques Correia, ${ }^{29}$ S. Henrot-Versille, ${ }^{113}$ F. Henry-Couannier, ${ }^{81}$ C. Hensel, ${ }^{53}$ T. Hen $\beta,{ }^{172}$ C. M. Hernandez, Y. Hernández Jiménez, ${ }^{165}$ R. Herrberg, ${ }^{15}$ A. D. Hershenhorn, ${ }^{150}$ G. Herten, ${ }^{47}$ R. Hertenberger,${ }^{96}$ L. Hervas,${ }^{29}$ G. G. Hesketh ${ }^{75}$ N. P. Hessey, ${ }^{103}$ E. Higón-Rodriguez, ${ }^{165}$ D. Hill, ${ }^{5, j j}$ J. C. Hill, ${ }^{27}$ N. Hill, ${ }^{5}$ K. H. Hiller, ${ }^{41}$ S. Hillert ${ }^{20}$ S. J. Hillier, ${ }^{17}$ I. Hinchliffe, ${ }^{14}$ E. Hines, ${ }^{118}$ M. Hirose, ${ }^{114}$ F. Hirsch, ${ }^{42}$ D. Hirschbuehl, ${ }^{172}$ J. Hobbs, ${ }^{146}$ N. Hod,${ }^{151}$ M. C. Hodgkinson, ${ }^{137}$ P. Hodgson, ${ }^{137}$ A. Hoecker,${ }^{29}$ M. R. Hoeferkamp, ${ }^{101}$ J. Hoffman, ${ }^{39}$ D. Hoffmann, ${ }^{81}$ M. Hohlfeld, ${ }^{79}$ M. Holder, ${ }^{139}$ S. O. Holmgren, ${ }^{14 a}$ T. Holy, ${ }^{125}$ J. L. Holzbauer, ${ }^{86}$ Y. Homma, ${ }^{65}$ T. M. Hong, ${ }^{118}$ L. Hooft van Huysduynen, ${ }^{106}$ T. Horazdovsky, ${ }^{125}$ C. Horn, ${ }^{141}$ S. Horner, ${ }^{47}$ J-Y. Hostachy, ${ }^{54}$ S. Hou,${ }^{149}$

M. A. Houlden, ${ }^{71}$ A. Hoummada, ${ }^{133 a}$ J. Howarth, ${ }^{80}$ D. F. Howell, ${ }^{116}$ I. Hristova,${ }^{15}$ J. Hrivnac, ${ }^{113}$ I. Hruska, ${ }^{123}$ T. Hryn'ova, ${ }^{4}$ P. J. Hsu, ${ }^{79}$ S.-C. Hsu, ${ }^{14}$ G. S. Huang,,${ }^{109}$ Z. Hubacek,${ }^{125}$ F. Hubaut,${ }^{81}$ F. Huegging,${ }^{20}$ A. Huettmann, ${ }^{41}$ T. B. Huffman, ${ }^{116}$ E. W. Hughes, ${ }^{34}$ G. Hughes, ${ }^{69}$ R. E. Hughes-Jones, ${ }^{80}$ M. Huhtinen, ${ }^{29}$ P. Hurst,${ }^{56}$ M. Hurwitz, ${ }^{14}$ 
U. Husemann, ${ }^{41}$ N. Huseynov, ${ }^{63, p}$ J. Huston, ${ }^{86}$ J. Huth, ${ }^{56}$ G. Iacobucci, ${ }^{48}$ G. Iakovidis, ${ }^{9}$ M. Ibbotson, ${ }^{80}$ I. Ibragimov, ${ }^{139}$ R. Ichimiya ${ }^{65} \mathrm{~L}$. Iconomidou-Fayard ${ }^{113} \mathrm{~J}$. Idarraga, ${ }^{113} \mathrm{P}$. Iengo, ${ }^{100 a}$ O. Igonkina, ${ }^{103} \mathrm{Y}$. Ikegami, ${ }^{64}$ M. Ikeno, ${ }^{64}$ Y. Ilchenko, ${ }^{39}$ D. Iliadis,${ }^{152} \mathrm{~N}$. Ilic, ${ }^{156} \mathrm{M}$. Imori, ${ }^{153}$ T. Ince, ${ }^{20} \mathrm{~J}$. Inigo-Golfin, ${ }^{29}$ P. Ioannou, ${ }^{8}$ M. Iodice, ${ }^{132 \mathrm{a}} \mathrm{V}$. Ippolito, ${ }^{130 \mathrm{a}, 130 \mathrm{~b}} \mathrm{~A}$. Irles Quiles, ${ }^{165} \mathrm{C}$. Isaksson, ${ }^{164} \mathrm{~A}$. Ishikawa, ${ }^{65} \mathrm{M}$. Ishino, ${ }^{66}$ R. Ishmukhametov ${ }^{39}$ C. Issever, ${ }^{16}$ S. Istin, ${ }^{18 a}$ A. V. Ivashin, ${ }^{126}$ W. Iwanski, ${ }^{38}$ H. Iwasaki, ${ }^{64} \mathrm{~J}$. M. Izen, ${ }^{40}$ V. Izzo, ${ }^{100 a}$ B. Jackson, ${ }^{18}$ J. N. Jackson, ${ }^{71}$ P. Jackson, ${ }^{141}$ M. R. Jaekel, ${ }^{29}$ V. Jain, ${ }^{59}$ K. Jakobs, ${ }^{47}$ S. Jakobsen, ${ }^{35}$ J. Jakubek, ${ }^{125}$ D. K. Jana, ${ }^{109}$ E. Jankowski, ${ }^{156}$ E. Jansen, ${ }^{75}$ H. Jansen, ${ }^{29}$ A. Jantsch, ${ }^{97}$ M. Janus, ${ }^{20}$ G. Jarlskog,${ }^{77}$ L. Jeanty, ${ }^{56}$ K. Jelen, ${ }^{37}$ I. Jen-La Plante, ${ }^{30}$ P. Jenni, ${ }^{29}$ A. Jeremie, ${ }^{4}$ P. Jež,${ }^{35}$ S. Jézéquel, ${ }^{4}$ M. K. Jha, ${ }^{19 a}$ H. Ji, ${ }^{10}$ W. Ji ${ }^{79}$ J. Jia, ${ }^{146}$ Y. Jiang, ${ }^{32 b}$ M. Jimenez Belenguer ${ }^{41}$ G. Jin, ${ }^{32 b}$ S. Jin, ${ }^{32 a}$ O. Jinnouchi, ${ }^{155}$ M. D. Joergensen, ${ }^{35}$ D. Joffe,${ }^{39}$ L. G. Johansen, ${ }^{13}$ M. Johansen, ${ }^{144 a, 144 b}$ K. E. Johansson, ${ }^{144 a}$ P. Johansson, ${ }^{137}$ S. Johnert, ${ }^{41}$ K. A. Johns, ${ }^{6}$ K. Jon-And,${ }^{144 a, 144 b}$ G. Jones, ${ }^{116}$ R. W. L. Jones, ${ }^{69}$ T. W. Jones, ${ }^{75}$ T. J. Jones, ${ }^{71}$ O. Jonsson, ${ }^{29}$ C. Joram, ${ }^{29}$ P. M. Jorge, ${ }^{122 \mathrm{a}}$ J. Joseph, ${ }^{14}$ J. Jovicevic, ${ }^{145}$ T. Jovin, ${ }^{12 \mathrm{~b}} \mathrm{X}$. Ju, ${ }^{170}$ C. A. Jung, ${ }^{42}$ R. M. Jungst,${ }^{29}$ V. Juranek,${ }^{123}$ P. Jussel, ${ }^{60}$ A. Juste Rozas, ${ }^{11}$ V. V. Kabachenko, ${ }^{126}$ S. Kabana,${ }^{16}$ M. Kaci, ${ }^{165}$ A. Kaczmarska, ${ }^{38}$ P. Kadlecik,${ }^{35}$ M. Kado, ${ }^{113}$ H. Kagan, ${ }^{107}$ M. Kagan,${ }^{56}$ S. Kaiser, ${ }^{97}$ E. Kajomovitz, ${ }^{150}$ S. Kalinin, ${ }^{172}$ L. V. Kalinovskaya, ${ }^{63}$ S. Kama, ${ }^{39}$ N. Kanaya, ${ }^{153}$ M. Kaneda, ${ }^{29}$ S. Kaneti, ${ }^{27}$ T. Kanno, ${ }^{155}$ V. A. Kantserov, ${ }^{94}$ J. Kanzaki, ${ }^{64}$ B. Kaplan, ${ }^{173}$ A. Kapliy, ${ }^{30}$ J. Kaplon, ${ }^{29}$ D. Kar, ${ }^{43}$ M. Karagounis, ${ }^{20}$ M. Karagoz, ${ }^{116}$ M. Karnevskiy, ${ }^{41}$ K. Karr, ${ }^{5}$ V. Kartvelishvilis, ${ }^{69}$ A. N. Karyukhin, ${ }^{126}$ L. Kashif, ${ }^{170}$ G. Kasieczka, ${ }^{57 b}$ R. D. Kass, ${ }^{107}$ A. Kastanas, ${ }^{13}$ M. Kataoka, ${ }^{4}$ Y. Kataoka, ${ }^{153}$

E. Katsoufis, ${ }^{9}$ J. Katzy, ${ }^{41}$ V. Kaushik,${ }^{6}$ K. Kawagoe, ${ }^{65}$ T. Kawamoto, ${ }^{153}$ G. Kawamura, ${ }^{79}$ M. S. Kayl, ${ }^{103}$ V. A. Kazanin, ${ }^{105}$ M. Y. Kazarinov, ${ }^{63}$ R. Keeler, ${ }^{167}$ R. Kehoe, ${ }^{39}$ M. Keil ${ }^{53}$ G. D. Kekelidze ${ }^{63}$ J. Kennedy,${ }^{96}$ C. J. Kenney, ${ }^{141}$ M. Kenyon, ${ }^{52}$ O. Kepka, ${ }^{123}$ N. Kerschen,${ }^{29}$ B. P. Kerševan, ${ }^{72}$ S. Kersten, ${ }^{172}$ K. Kessoku, ${ }^{153}$ J. Keung, ${ }^{156}$ F. Khalil-zada, ${ }^{10}$ H. Khandanyan, ${ }^{163}$ A. Khanov, ${ }^{110}$ D. Kharchenko, ${ }^{63}$ A. Khodinov, ${ }^{94}$ A. G. Kholodenko, ${ }^{126}$ A. Khomich, ${ }^{57 \mathrm{a}}$ T. J. Khoo, ${ }^{27}$ G. Khoriauli, ${ }^{20}$ A. Khoroshilov, ${ }^{172}$ N. Khovanskiy, ${ }^{63}$ V. Khovanskiy, ${ }^{93}$ E. Khramov, ${ }^{63}$ J. Khubua, ${ }^{50 b}$ H. Kim,,${ }^{144 a, 144 b}$ M. S. Kim, ${ }^{2}$ S. H. Kim, ${ }^{158}$ N. Kimura, ${ }^{168}$ O. Kind, ${ }^{15}$ B. T. King, ${ }^{71}$ M. King, ${ }^{65}$ R. S. B. King,,${ }^{16}$ J. Kirk, ${ }^{127}$ L. E. Kirsch, ${ }^{22}$ A. E. Kiryunin, ${ }^{97}$ T. Kishimoto, ${ }^{65}$ D. Kisielewska, ${ }^{37}$ T. Kittelmann, ${ }^{121}$ A. M. Kiver, ${ }^{126}$ E. Kladiva, ${ }^{142 b}$ J. Klaiber-Lodewigs, ${ }^{42}$ M. Klein, ${ }^{71}$ U. Klein, ${ }^{71}$ K. Kleinknecht ${ }^{79}$ M. Klemetti, ${ }^{83}$ A. Klier, ${ }^{169}$ P. Klimek, ${ }^{144 a, 144 b}$ A. Klimentov, ${ }^{24}$ R. Klingenberg, ${ }^{42}$ J. A. Klinger, ${ }^{80}$ E. B. Klinkby, ${ }^{35}$ T. Klioutchnikova, ${ }^{29}$ P. F. Klok, ${ }^{102}$ S. Klous, ${ }^{103}$ E.-E. Kluge,${ }^{57 a}$ T. Kluge, ${ }^{71}$ P. Kluit, ${ }^{103}$ S. Kluth, ${ }^{97}$ N. S. Knecht, ${ }^{156}$ E. Kneringer ${ }^{60}$ J. Knobloch, ${ }^{29}$ E. B. F. G. Knoops, ${ }^{81}$ A. Knue, ${ }^{53}$ B. R. Ko, ${ }^{44}$ T. Kobayashi, ${ }^{153}$ M. Kobel, ${ }^{43}$ M. Kocian, ${ }^{141}$ P. Kodys, ${ }^{124}$ K. Köneke, ${ }^{29}$ A. C. König, ${ }^{102}$ S. Koenig, ${ }^{79}$ L. Köpke,${ }^{79}$ F. Koetsveld,${ }^{102}$ P. Koevesarki ${ }^{20}$ T. Koffas,${ }^{28}$ E. Koffeman, ${ }^{103}$ L. A. Kogan,${ }^{116}$ F. Kohn, ${ }^{53}$ Z. Kohout, ${ }^{125}$ T. Kohriki, ${ }^{64}$ T. Koi ${ }^{141}$

T. Kokott, ${ }^{20}$ G. M. Kolachev, ${ }^{105}$ H. Kolanoski, ${ }^{15}$ V. Kolesnikov,${ }^{63}$ I. Koletsou, ${ }^{87 a}$ J. Koll ${ }^{86}$ M. Kollefrath,${ }^{47}$

S. D. Kolya ${ }^{80}$ A. A. Komar, ${ }^{92}$ Y. Komori, ${ }^{153}$ T. Kondo, ${ }^{64}$ T. Kono, ${ }^{41, q}$ A. I. Kononov ${ }^{47}$ R. Konoplich,,${ }^{106, r}$ N. Konstantinidis, ${ }^{75}$ A. Kootz, ${ }^{172}$ S. Koperny, ${ }^{37}$ K. Korcyl, ${ }^{38}$ K. Kordas, ${ }^{152}$ V. Koreshev, ${ }^{126}$ A. Korn, ${ }^{116}$ A. Korol, ${ }^{105}$ I. Korolkov, ${ }^{11}$ E. V. Korolkova, ${ }^{137}$ V. A. Korotkov, ${ }^{126}$ O. Kortner, ${ }^{97}$ S. Kortner, ${ }^{97}$ V. V. Kostyukhin, ${ }^{20}$

M. J. Kotamäki, ${ }^{29}$ S. Kotov, ${ }^{97}$ V. M. Kotov, ${ }^{63}$ A. Kotwal,${ }^{44}$ C. Kourkoumelis, ${ }^{8}$ V. Kouskoura, ${ }^{152}$ A. Koutsman, ${ }^{157 a}$ R. Kowalewski, ${ }^{167}$ T.Z. Kowalski, ${ }^{37}$ W. Kozanecki, ${ }^{134}$ A. S. Kozhin, ${ }^{126}$ V. Kral, ${ }^{125}$ V. A. Kramarenko, ${ }^{95}$

G. Kramberger, ${ }^{72}$ M. W. Krasny, ${ }^{76}$ A. Krasznahorkay, ${ }^{106}$ J. Kraus, ${ }^{86}$ J. K. Kraus,${ }^{20}$ A. Kreisel, ${ }^{151}$ F. Krejci, ${ }^{125}$ J. Kretzschmar, ${ }^{71}$ N. Krieger, ${ }^{53}$ P. Krieger, ${ }^{156}$ K. Kroeninger, ${ }^{53}$ H. Kroha,${ }^{97}$ J. Kroll, ${ }^{118}$ J. Kroseberg, ${ }^{20}$ J. Krstic, ${ }^{12 a}$ U. Kruchonak ${ }^{63}$ H. Krüger ${ }^{20}$ T. Kruker, ${ }^{16}$ N. Krumnack, ${ }^{62}$ Z. V. Krumshteyn, ${ }^{63}$ A. Kruth, ${ }^{20}$ T. Kubota, ${ }^{84}$ S. Kuday, ${ }^{3 a}$ S. Kuehn, ${ }^{47}$ A. Kugel,${ }^{57 \mathrm{c}}$ T. Kuhl,${ }^{41}$ D. Kuhn, ${ }^{60}$ V. Kukhtin, ${ }^{63}$ Y. Kulchitsky, ${ }^{88}$ S. Kuleshov, ${ }^{31 \mathrm{~b}}$ C. Kummer, ${ }^{96}$ M. Kuna ${ }^{76}$ N. Kundu, ${ }^{116}$ J. Kunkle, ${ }^{118}$ A. Kupco, ${ }^{123}$ H. Kurashige,${ }^{65}$ M. Kurata, ${ }^{158}$ Y. A. Kurochkin,${ }^{88}$ V. Kus, ${ }^{123}$ E. S. Kuwertz, ${ }^{145}$ M. Kuze, ${ }^{155}$ J. Kvita, ${ }^{140}$ R. Kwee, ${ }^{15}$ A. La Rosa,${ }^{48}$ L. La Rotonda, ${ }^{36 a, 36 b}$ L. Labarga, ${ }^{78}$ J. Labbe, ${ }^{4}$ S. Lablak, ${ }^{133 a}$ C. Lacasta, ${ }^{165}$ F. Lacava, ${ }^{130 a, 130 b}$ H. Lacker,${ }^{15}$ D. Lacour,${ }^{76}$ V. R. Lacuesta, ${ }^{165}$ E. Ladygin, ${ }^{63}$ R. Lafaye, ${ }^{4}$ B. Laforge ${ }^{76}$ T. Lagouri, ${ }^{78}$ S. Lai, ${ }^{47}$ E. Laisne,${ }^{54}$ M. Lamanna, ${ }^{29}$ C. L. Lampen, ${ }^{6}$ W. Lampl,${ }^{6}$ E. Lancon, ${ }^{134}$ U. Landgraf, ${ }^{47}$ M. P. J. Landon, ${ }^{73}$ J. L. Lane, ${ }^{80}$ C. Lange, ${ }^{41}$ A. J. Lankford, ${ }^{161}$ F. Lanni, ${ }^{24}$

K. Lantzsch, ${ }^{172}$ S. Laplace, ${ }^{76}$ C. Lapoire, ${ }^{20}$ J. F. Laporte, ${ }^{134}$ T. Lari, ${ }^{87 a}$ A. V. Larionov, ${ }^{126}$ A. Larner, ${ }^{116}$ C. Lasseur, ${ }^{29}$ M. Lassnig, ${ }^{29}$ P. Laurelli, ${ }^{46}$ V. Lavorini, ${ }^{36 a, 36 b}$ W. Lavrijsen, ${ }^{14}$ P. Laycock, ${ }^{71}$ A. B. Lazarev, ${ }^{63}$ O. Le Dortz, ${ }^{76}$ E. Le Guirriec, ${ }^{81}$ C. Le Maner, ${ }^{156}$ E. Le Menedeu, ${ }^{9}$ C. Lebel,${ }^{91}$ T. LeCompte, ${ }^{5}$ F. Ledroit-Guillon,${ }^{54}$ H. Lee, ${ }^{103}$ J. S. H. Lee, ${ }^{114}$ S. C. Lee, ${ }^{149}$ L. Lee, ${ }^{173}$ M. Lefebvre, ${ }^{167}$ M. Legendre, ${ }^{134}$ A. Leger, ${ }^{48}$ B. C. LeGeyt, ${ }^{118}$ F. Legger, ${ }^{96}$ C. Leggett, ${ }^{14}$ M. Lehmacher ${ }^{20}$ G. Lehmann Miotto, ${ }^{29}$ X. Lei, ${ }^{6}$ M. A. L. Leite, ${ }^{23 d}$ R. Leitner, ${ }^{124}$ D. Lellouch ${ }^{169}$ M. Leltchouk, ${ }^{34}$ B. Lemmer, ${ }^{53}$ V. Lendermann, ${ }^{57 a}$ K. J. C. Leney, ${ }^{143 b}$ T. Lenz, ${ }^{103}$ G. Lenzen, ${ }^{172}$ B. Lenzi, ${ }^{29}$ 
K. Leonhardt, ${ }^{43}$ S. Leontsinis, ${ }^{9}$ C. Leroy, ${ }^{91}$ J-R. Lessard, ${ }^{167}$ J. Lesser, ${ }^{144 a}$ C. G. Lester, ${ }^{27}$ A. Leung Fook Cheong, ${ }^{170}$ J. Levêque, ${ }^{4}$ D. Levin, ${ }^{85}$ L. J. Levinson, ${ }^{169}$ M. S. Levitski, ${ }^{126}$ A. Lewis, ${ }^{116}$ G. H. Lewis, ${ }^{106}$ A. M. Leyko, ${ }^{20}$ M. Leyton, ${ }^{15}$ B. Li ${ }^{81} \mathrm{H}$. Li ${ }^{170, s}$ S. Li ${ }^{32 b, t}$ X. Li ${ }^{85}$ Z. Liang, ${ }^{116, \mathrm{u}} \mathrm{H}$. Liao, ${ }^{33}$ B. Liberti, ${ }^{131 \mathrm{a}}$ P. Lichard, ${ }^{29}$ M. Lichtnecker, ${ }^{96}$ K. Lie, ${ }^{163}$ W. Liebig, ${ }^{13}$ R. Lifshitz, ${ }^{150}$ C. Limbach,${ }^{20}$ A. Limosani,${ }^{84}$ M. Limper, ${ }^{61}$ S. C. Lin, ${ }^{149, v}$ F. Linde, ${ }^{103}$ J. T. Linnemann, ${ }^{86}$ E. Lipeles, ${ }^{18}$ L. Lipinsky, ${ }^{123}$ A. Lipniacka, ${ }^{13}$ T. M. Liss, ${ }^{163}$ D. Lissauer,${ }^{24}$ A. Lister,${ }^{48}$ A. M. Litke, ${ }^{135}$ C. Liu, ${ }^{28}$ D. Liu,${ }^{149}$ H. Liu, ${ }^{85}$ J. B. Liu ${ }^{85}$ M. Liu, ${ }^{32 b}$ Y. Liu, ${ }^{32 b}$ M. Livan, ${ }^{117 a, 117 b}$ S. S. A. Livermore, ${ }^{116}$ A. Lleres,${ }^{54}$ J. Llorente Merino, ${ }^{78}$ S. L. Lloyd, ${ }^{73}$ E. Lobodzinska, ${ }^{41}$ P. Loch, ${ }^{6}$ W. S. Lockman, ${ }^{135}$ T. Loddenkoetter ${ }^{20}$ F. K. Loebinger, ${ }^{80}$ A. Loginov, ${ }^{173}$ C. W. Loh, ${ }^{166}$ T. Lohse, ${ }^{15}$ K. Lohwasser ${ }^{47}$ M. Lokajicek, ${ }^{123}$ J. Loken, ${ }^{116}$ V. P. Lombardo, ${ }^{4}$ R. E. Long, ${ }^{69}$ L. Lopes, ${ }^{122 a}$ D. Lopez Mateos, ${ }^{56}$ J. Lorenz,${ }^{96}$ N. Lorenzo Martinez, ${ }^{113}$ M. Losada, ${ }^{160}$ P. Loscutoff, ${ }^{14}$ F. Lo Sterzo, ${ }^{130 a, 130 b}$ M. J. Losty, ${ }^{157 a}$ X. Lou, ${ }^{40}$ A. Lounis, ${ }^{113}$ K. F. Loureiro, ${ }^{160}$ J. Love,${ }^{21}$ P. A. Love, ${ }^{69}$ A. J. Lowe,${ }^{141, e}$ F. Lu, ${ }^{32 a}$ H. J. Lubatti, ${ }^{136}$ C. Luci, ${ }^{130 a, 130 b}$ A. Lucotte,${ }^{54}$ A. Ludwig, ${ }^{43}$ D. Ludwig, ${ }^{41}$ I. Ludwig, ${ }^{47}$ J. Ludwig, ${ }^{47}$ F. Luehring, ${ }^{59}$ G. Luijckx,${ }^{103}$ D. Lumb,${ }^{47}$ L. Luminari, ${ }^{130 a}$ E. Lund, ${ }^{115}$ B. Lund-Jensen, ${ }^{145}$ B. Lundberg, ${ }^{77}$ J. Lundberg, ${ }^{144 a, 144 b}$ J. Lundquist, ${ }^{35}$ M. Lungwitz, ${ }^{79}$ G. Lutz, ${ }^{97}$ D. Lynn, ${ }^{24}$ J. Lys, ${ }^{14}$ E. Lytken, ${ }^{77}$ H. Ma,${ }^{24}$ L. L. Ma,${ }^{170}$ J. A. Macana Goia,${ }^{91}$ G. Maccarrone, ${ }^{46}$ A. Macchiolo, ${ }^{97}$ B. Maček,${ }^{72}$ J. Machado Miguens, ${ }^{122 a}$ R. Mackeprang, ${ }^{35}$ R. J. Madaras, ${ }^{14}$ W. F. Mader, ${ }^{43}$ R. Maenner, ${ }^{57 \mathrm{c}}$ T. Maeno, ${ }^{24}$ P. Mättig, ${ }^{172}$ S. Mättig, ${ }^{41}$ L. Magnoni, ${ }^{29}$ E. Magradze,${ }^{53}$ Y. Mahalalel,${ }^{151}$ K. Mahboubi, ${ }^{47}$ G. Mahout, ${ }^{17}$

C. Maiani, ${ }^{130 a, 130 b}$ C. Maidantchik, ${ }^{23 a}$ A. Maio, ${ }^{122 a, b}$ S. Majewski, ${ }^{24}$ Y. Makida,${ }^{64}$ N. Makovec, ${ }^{113}$ P. Mal, ${ }^{134}$ B. Malaescu, ${ }^{29}$ Pa. Malecki, ${ }^{38}$ P. Malecki, ${ }^{38}$ V. P. Maleev, ${ }^{119}$ F. Malek, ${ }^{54}$ U. Mallik, ${ }^{61}$ D. Malon, ${ }^{5}$ C. Malone,${ }^{141}$ S. Maltezos, ${ }^{9}$ V. Malyshev ${ }^{105}$ S. Malyukov, ${ }^{29}$ R. Mameghani, ${ }^{96}$ J. Mamuzic, ${ }^{12 b}$ A. Manabe,${ }^{64}$ L. Mandelli, ${ }^{87 a}$ I. Mandic,${ }^{72}$ R. Mandrysch, ${ }^{15}$ J. Maneira, ${ }^{122 a}$ P. S. Mangeard, ${ }^{86}$ L. Manhaes de Andrade Filho, ${ }^{23 a}$ I. D. Manjavidze, ${ }^{63}$ A. Mann, ${ }^{53}$ P. M. Manning, ${ }^{135}$ A. Manousakis-Katsikakis, ${ }^{8}$ B. Mansoulie, ${ }^{134}$ A. Manz, ${ }^{97}$ A. Mapelli, ${ }^{29}$ L. Mapelli, ${ }^{29}$ L. March ${ }^{78}$ J. F. Marchand ${ }^{28}$ F. Marchese, ${ }^{131 \mathrm{a}, 131 \mathrm{~b}}$ G. Marchiori, ${ }^{76}$ M. Marcisovsky, ${ }^{123}$ A. Marin, ${ }^{21, j \mathrm{j}}$ C. P. Marino, ${ }^{167}$ F. Marroquim, ${ }^{23 a}$ R. Marshall, ${ }^{80}$ Z. Marshall,,${ }^{29}$ F. K. Martens, ${ }^{156}$ S. Marti-Garcia, ${ }^{165}$ A. J. Martin, ${ }^{173}$ B. Martin, ${ }^{29}$ B. Martin, ${ }^{86}$ F. F. Martin, ${ }^{118}$ J. P. Martin, ${ }^{91}$ Ph. Martin, ${ }^{54}$ T. A. Martin, ${ }^{17}$ V. J. Martin, ${ }^{45}$

B. Martin dit Latour, ${ }^{48}$ S. Martin-Haugh, ${ }^{147}$ M. Martinez,${ }^{11}$ V. Martinez Outschoorn, ${ }^{56}$ A. C. Martyniuk,,${ }^{167}$ M. Marx ${ }^{80}$ F. Marzano, ${ }^{130 a}$ A. Marzin, ${ }^{109}$ L. Masetti ${ }^{79}$ T. Mashimo, ${ }^{153}$ R. Mashinistov, ${ }^{92}$ J. Masik, ${ }^{80}$ A. L. Maslennikov, ${ }^{105}$ I. Massa, ${ }^{19 a, 19 b}$ G. Massaro, ${ }^{103}$ N. Massol, ${ }^{4}$ P. Mastrandrea, ${ }^{130 a, 130 b}$ A. Mastroberardino, ${ }^{36 a, 36 b}$ T. Masubuchi, ${ }^{153}$ M. Mathes,${ }^{20}$ P. Matricon, ${ }^{113}$ H. Matsumoto, ${ }^{153}$ H. Matsunaga, ${ }^{153}$ T. Matsushita, ${ }^{65}$

C. Mattravers, ${ }^{116, \mathrm{c}}$ J. M. Maugain, ${ }^{29}$ J. Maurer, ${ }^{81}$ S. J. Maxfield, ${ }^{71}$ D. A. Maximov, ${ }^{105, f}$ E. N. May, ${ }^{5}$ A. Mayne, ${ }^{137}$ R. Mazini, ${ }^{149}$ M. Mazur, ${ }^{20}$ M. Mazzanti, ${ }^{87 a}$ E. Mazzoni, ${ }^{120 a, 120 b}$ S. P. Mc Kee ${ }^{85}$ A. McCarn, ${ }^{163}$ R. L. McCarthy, ${ }^{146}$ T. G. McCarthy, ${ }^{28}$ N. A. McCubbin, ${ }^{127}$ K. W. McFarlane, ${ }^{55}$ J. A. Mcfayden,,${ }^{137}$ H. McGlone, ${ }^{52}$ G. Mchedlidze,${ }^{50 b}$ R. A. McLaren, ${ }^{29}$ T. Mclaughlan, ${ }^{17}$ S. J. McMahon, ${ }^{127}$ R. A. McPherson, ${ }^{167, j}$ A. Meade, ${ }^{82}$ J. Mechnich, ${ }^{103}$ M. Mechtel, ${ }^{172}$ M. Medinnis, ${ }^{41}$ R. Meera-Lebbai, ${ }^{109}$ T. Meguro, ${ }^{114}$ R. Mehdiyev, ${ }^{91}$ S. Mehlhase, ${ }^{35}$ A. Mehta,${ }^{71}$

K. Meier, ${ }^{57 a}$ B. Meirose,${ }^{77}$ C. Melachrinos, ${ }^{30}$ B. R. Mellado Garcia,,${ }^{170}$ L. Mendoza Navas, ${ }^{160}$ Z. Meng,,${ }^{149, s}$ A. Mengarelli, ${ }^{19 a, 19 b}$ S. Menke, ${ }^{97}$ C. Menot, ${ }^{29}$ E. Meoni, ${ }^{11}$ K. M. Mercurio,${ }^{56}$ P. Mermod,${ }^{48}$ L. Merola, ${ }^{100 a, 100 b}$ C. Meroni, ${ }^{87 a}$ F. S. Merritt, ${ }^{30}$ H. Merritt, ${ }^{107}$ A. Messina,${ }^{29}$ J. Metcalfe, ${ }^{101}$ A. S. Mete, ${ }^{62}$ C. Meyer, ${ }^{79}$ C. Meyer, ${ }^{30}$ J-P. Meyer, ${ }^{134}$ J. Meyer, ${ }^{171}$ J. Meyer, ${ }^{53}$ T. C. Meyer, ${ }^{29}$ W. T. Meyer, ${ }^{62}$ J. Miao, ${ }^{32 d}$ S. Michal, ${ }^{29}$ L. Micu, ${ }^{25 a}$ R. P. Middleton, ${ }^{127}$ S. Migas, ${ }^{71}$ L. Mijović, ${ }^{41}$ G. Mikenberg, ${ }^{169}$ M. Mikestikova, ${ }^{123}$ M. Mikuž, ${ }^{72}$ D. W. Miller,${ }^{30}$ R. J. Miller, ${ }^{86}$ W. J. Mills, ${ }^{166}$ C. Mills,${ }^{56}$ A. Milov, ${ }^{169}$ D. A. Milstead,${ }^{144 a, 144 b}$ D. Milstein, ${ }^{169}$ A. A. Minaenko, ${ }^{126}$ M. Miñano Moya, ${ }^{165}$ I. A. Minashvili, ${ }^{63}$ A. I. Mincer, ${ }^{106}$ B. Mindur,${ }^{37}$ M. Mineev, ${ }^{63}$ Y. Ming, ${ }^{170}$ L. M. Mir, ${ }^{11}$ G. Mirabelli, ${ }^{130 a}$ L. Miralles Verge, ${ }^{11}$ A. Misiejuk, ${ }^{74}$ J. Mitrevski, ${ }^{135}$ G. Y. Mitrofanov, ${ }^{126}$ V. A. Mitsou, ${ }^{165}$ S. Mitsui ${ }^{64}$ P. S. Miyagawa, ${ }^{137}$ K. Miyazaki, ${ }^{65}$ J. U. Mjörnmark, ${ }^{77}$ T. Moa, ${ }^{144 a, 144 b}$ P. Mockett, ${ }^{136}$ S. Moed, ${ }^{56}$ V. Moeller, ${ }^{27}$ K. Mönig, ${ }^{41}$ N. Möser, ${ }^{20}$ S. Mohapatra, ${ }^{146}$ W. Mohr, ${ }^{47}$ S. Mohrdieck-Möck, ${ }^{97}$ A. M. Moisseev, ${ }^{126, j j}$

R. Moles-Valls, ${ }^{165}$ J. Molina-Perez, ${ }^{29}$ J. Monk, ${ }^{75}$ E. Monnier, ${ }^{81}$ S. Montesano,${ }^{87 a, 87 b}$ F. Monticelli, ${ }^{68}$ S. Monzani, ${ }^{19 a, 19 b}$ R. W. Moore, ${ }^{2}$ G. F. Moorhead ${ }^{84}$ C. Mora Herrera ${ }^{48}$ A. Moraes ${ }^{52}$ N. Morange, ${ }^{134}$ J. Morel,${ }^{53}$ G. Morello, ${ }^{36 a, 36 b}$ D. Moreno, ${ }^{79}$ M. Moreno Llácer, ${ }^{165}$ P. Morettini ${ }^{49 a}$ M. Morgenstern, ${ }^{43}$ M. Morii, ${ }^{56}$ J. Morin,${ }^{73}$ A. K. Morley, ${ }^{29}$ G. Mornacchi, ${ }^{29}$ S. V. Morozov, ${ }^{94}$ J. D. Morris, ${ }^{73}$ L. Morvaj, ${ }^{99}$ H. G. Moser, ${ }^{97}$ M. Mosidze, ${ }^{50 b}$ J. Moss, ${ }^{107}$ R. Mount,${ }^{141}$ E. Mountricha, ${ }^{9, w}$ S. V. Mouraviev, ${ }^{92}$ E. J. W. Moyse, ${ }^{82}$ M. Mudrinic,${ }^{12 b}$ F. Mueller,${ }^{57 a}$ J. Mueller, ${ }^{121}$ K. Mueller, ${ }^{20}$ T. A. Müller, ${ }^{96}$ T. Mueller, ${ }^{79}$ D. Muenstermann, ${ }^{29}$ A. Muir, ${ }^{166}$ Y. Munwes, ${ }^{151}$ W. J. Murray, ${ }^{127}$ I. Mussche, ${ }^{103}$ E. Musto, ${ }^{100 a, 100 b}$ A. G. Myagkov, ${ }^{126}$ M. Myska, ${ }^{123}$ J. Nadal, ${ }^{11}$ K. Nagai, ${ }^{158}$ K. Nagano, ${ }^{64}$ A. Nagarkar, ${ }^{107}$ Y. Nagasaka, ${ }^{58}$ M. Nagel, ${ }^{97}$ A. M. Nairz,${ }^{29}$ Y. Nakahama, ${ }^{29}$ K. Nakamura, ${ }^{153}$ 
T. Nakamura, ${ }^{153}$ I. Nakano, ${ }^{108}$ G. Nanava,${ }^{20}$ A. Napier,${ }^{159}$ R. Narayan,${ }^{57 b}$ M. Nash, ${ }^{75, c}$ N. R. Nation, ${ }^{21}$ T. Nattermann, ${ }^{20}$ T. Naumann, ${ }^{41}$ G. Navarro, ${ }^{160}$ H. A. Neal, ${ }^{85}$ E. Nebot,${ }^{78}$ P. Yu. Nechaeva, ${ }^{92}$ T. J. Neep,${ }^{80}$ A. Negri, ${ }^{117 a, 117 b}$ G. Negri, ${ }^{29}$ S. Nektarijevic, ${ }^{48}$ A. Nelson, ${ }^{161}$ S. Nelson, ${ }^{141}$ T. K. Nelson, ${ }^{141}$ S. Nemecek, ${ }^{123}$ P. Nemethy, ${ }^{106}$ A. A. Nepomuceno, ${ }^{23 a}$ M. Nessi, ${ }^{29, x}$ M. S. Neubauer, ${ }^{163}$ A. Neusiedl, ${ }^{79}$ R. M. Neves, ${ }^{106}$ P. Nevski,${ }^{24}$ P. R. Newman, ${ }^{17}$ V. Nguyen Thi Hong, ${ }^{134}$ R. B. Nickerson,,${ }^{116}$ R. Nicolaidou, ${ }^{134}$ L. Nicolas, ${ }^{137}$ B. Nicquevert, ${ }^{29}$ F. Niedercorn, ${ }^{13}$ J. Nielsen, ${ }^{135}$ T. Niinikoski, ${ }^{29}$ N. Nikiforou, ${ }^{34}$ A. Nikiforov, ${ }^{15}$ V. Nikolaenko, ${ }^{126}$ K. Nikolaev, ${ }^{63}$ I. Nikolic-Audit, ${ }^{76}$ K. Nikolics, ${ }^{48}$ K. Nikolopoulos, ${ }^{24}$ H. Nilsen, ${ }^{47}$ P. Nilsson, ${ }^{7}$ Y. Ninomiya, ${ }^{153}$ A. Nisati, ${ }^{130 a}$ T. Nishiyama, ${ }^{65}$ R. Nisius, ${ }^{97}$ L. Nodulman, ${ }^{5}$ M. Nomachi, ${ }^{114}$ I. Nomidis, ${ }^{152}$ M. Nordberg, ${ }^{29}$ B. Nordkvist,${ }^{144 a, 144 b}$ P. R. Norton, ${ }^{127}$ J. Novakova, ${ }^{124}$ M. Nozaki ${ }^{64}$ L. Nozka, ${ }^{111}$ I. M. Nugent, ${ }^{157 a}$ A.-E. Nuncio-Quiroz, ${ }^{20}$ G. Nunes Hanninger, ${ }^{84}$ T. Nunnemann, ${ }^{96}$ E. Nurse, ${ }^{75}$ B. J. O’Brien, ${ }^{45}$ S. W. O'Neale, ${ }^{17, j j}$ D. C. O'Neil, ${ }^{140}$ V. O'Shea,${ }^{52}$ L. B. Oakes, ${ }^{96}$ F. G. Oakham ${ }^{28, d}$ H. Oberlack,${ }^{97}$ J. Ocariz,${ }^{76}$ A. Ochi, ${ }^{65}$ S. Oda,${ }^{153}$ S. Odaka ${ }^{64}$ J. Odier, ${ }^{81}$ H. Ogren,${ }^{59}$ A. Oh ${ }^{80}$ S. H. Oh,${ }^{44}$ C. C. Ohm, ${ }^{144 a, 144 b}$ T. Ohshima, ${ }^{99}$ H. Ohshita, ${ }^{138}$ T. Ohsugi, ${ }^{176}$ S. Okada,${ }^{65}$ H. Okawa, ${ }^{161}$ Y. Okumura, ${ }^{99}$ T. Okuyama, ${ }^{153}$ A. Olariu, ${ }^{25 a}$ M. Olcese,${ }^{49 a}$ A. G. Olchevski, ${ }^{63}$ S. A. Olivares Pino, ${ }^{31 \mathrm{a}}$ M. Oliveira, ${ }^{122 a, \mathrm{~h}}$ D. Oliveira Damazio, ${ }^{24}$ E. Oliver Garcia,${ }^{165}$ D. Olivito, ${ }^{118}$ A. Olszewski, ${ }^{38}$ J. Olszowska, ${ }^{38}$ C. Omachi, ${ }^{65}$ A. Onofre, ${ }^{122 a, y}$ P. U.E. Onyisi, ${ }^{30}$ C. J. Oram,${ }^{157 a}$ M. J. Oreglia, ${ }^{30}$ Y. Oren,,${ }^{151}$

D. Orestano, ${ }^{132 a, 132 b}$ I. Orlov, ${ }^{105}$ C. Oropeza Barrera, ${ }^{52}$ R. S. Orr, ${ }^{156}$ B. Osculati, ${ }^{49 a, 49 b}$ R. Ospanov, ${ }^{118}$ C. Osuna, ${ }^{11}$ G. Otero y Garzon, ${ }^{26}$ J. P. Ottersbach, ${ }^{103}$ M. Ouchrif, ${ }^{133 d}$ E. A. Ouellette, ${ }^{167}$ F. Ould-Saada, ${ }^{115}$ A. Ouraou, ${ }^{134}$ Q. Ouyang, ${ }^{32 a}$ A. Ovcharova, ${ }^{14}$ M. Owen, ${ }^{80}$ S. Owen, ${ }^{137}$ V.E. Ozcan, ${ }^{18 a}$ N. Ozturk, ${ }^{7}$ A. Pacheco Pages,${ }^{11}$ C. Padilla Aranda ${ }^{11}$ S. Pagan Griso, ${ }^{14}$ E. Paganis, ${ }^{137}$ F. Paige, ${ }^{24}$ P. Pais,${ }^{82}$ K. Pajchel, ${ }^{115}$ G. Palacino, ${ }^{157 b}$ C. P. Paleari, ${ }^{6}$ S. Palestini, ${ }^{29}$ D. Pallin,${ }^{33}$ A. Palma, ${ }^{122 a}$ J. D. Palmer, ${ }^{17}$ Y. B. Pan,${ }^{170}$ E. Panagiotopoulou, ${ }^{9}$ B. Panes,${ }^{31 a}$ N. Panikashvili, ${ }^{85}$ S. Panitkin, ${ }^{24}$ D. Pantea, ${ }^{25 a}$ M. Panuskova, ${ }^{123}$ V. Paolone, ${ }^{121}$ A. Papadelis, ${ }^{14 a}$

Th. D. Papadopoulou, ${ }^{9}$ A. Paramonov,${ }^{5}$ D. Paredes Hernandez,${ }^{33}$ W. Park, ${ }^{24, z}$ M. A. Parker,${ }^{27}$ F. Parodi, ${ }^{49 a, 49 b}$ J. A. Parsons, ${ }^{34}$ U. Parzefall, ${ }^{47}$ E. Pasqualucci, ${ }^{130 a}$ S. Passaggio, ${ }^{49 a}$ A. Passeri, ${ }^{132 a}$ F. Pastore, ${ }^{132 a, 132 b}$ Fr. Pastore ${ }^{74}$ G. Pásztor, ${ }^{48, a a}$ S. Pataraia, ${ }^{172}$ N. Patel, ${ }^{148}$ J. R. Pater, ${ }^{80}$ S. Patricelli, ${ }^{100 a, 100 b}$ T. Pauly, ${ }^{29}$ M. Pecsy, ${ }^{142 a}$

M. I. Pedraza Morales, ${ }^{170}$ S. V. Peleganchuk, ${ }^{105}$ H. Peng, ${ }^{32 b}$ R. Pengo, ${ }^{29}$ B. Penning, ${ }^{30}$ A. Penson, ${ }^{34}$ J. Penwell, ${ }^{59}$ M. Perantoni, ${ }^{23 a}$ K. Perez,${ }^{34, b b}$ T. Perez Cavalcanti, ${ }^{41}$ E. Perez Codina, ${ }^{11}$ M. T. Pérez García-Estañ, ${ }^{165}$ V. Perez Reale, ${ }^{34}$ L. Perini,${ }^{87 a, 87 b}$ H. Pernegger, ${ }^{29}$ R. Perrino, ${ }^{70 a}$ P. Perrodo,${ }^{4}$ S. Persembe, ${ }^{3 a}$ A. Perus,${ }^{113}$ V. D. Peshekhonov, ${ }^{63}$ K. Peters,${ }^{29}$ B. A. Petersen, ${ }^{29}$ J. Petersen, ${ }^{29}$ T. C. Petersen, ${ }^{35}$ E. Petit, ${ }^{4}$ A. Petridis, ${ }^{152}$ C. Petridou, ${ }^{152}$ E. Petrolo, ${ }^{130 \mathrm{a}}$ F. Petrucci, ${ }^{132 \mathrm{a}, 132 \mathrm{~b}}$ D. Petschull, ${ }^{41}$ M. Petteni,${ }^{140}$ R. Pezoa,${ }^{31 \mathrm{~b}}$ A. Phan, ${ }^{84}$ P. W. Phillips, ${ }^{127}$ G. Piacquadio, ${ }^{29}$ E. Piccaro,${ }^{73}$ M. Piccinini, ${ }^{19 a, 19 b}$ S. M. Piec, ${ }^{41}$ R. Piegaia, ${ }^{26}$ D. T. Pignotti,${ }^{107}$ J.E. Pilcher ${ }^{30}$ A. D. Pilkington, ${ }^{80}$ J. Pina, ${ }^{122 a, b}$ M. Pinamonti, ${ }^{162 a, 162 c}$ A. Pinder, ${ }^{116}$ J. L. Pinfold, ${ }^{2}$ J. Ping, ${ }^{32 c}$ B. Pinto, ${ }^{122 \mathrm{a}}$ O. Pirotte, ${ }^{29}$ C. Pizio, ${ }^{87 \mathrm{a}, 87 \mathrm{~b}}$ M. Plamondon, ${ }^{167}$ M.-A. Pleier ${ }^{24}$ A. V. Pleskach,${ }^{126}$ A. Poblaguev, ${ }^{24}$ S. Poddar, ${ }^{57 a}$ F. Podlyski, ${ }^{33}$ L. Poggioli, ${ }^{13}$ T. Poghosyan,${ }^{20}$ M. Pohl,${ }^{48}$ F. Polci,${ }^{54}$ G. Polesello, ${ }^{117 a}$

A. Policicchio, ${ }^{36 a, 36 b}$ A. Polini, ${ }^{19 a}$ J. Poll, ${ }^{73}$ V. Polychronakos, ${ }^{24}$ D. M. Pomarede, ${ }^{134}$ D. Pomeroy, ${ }^{22}$ K. Pommès, ${ }^{29}$ L. Pontecorvo, ${ }^{130 a}$ B. G. Pope,${ }^{86}$ G. A. Popeneciu, ${ }^{25 a}$ D. S. Popovic, ${ }^{12 a}$ A. Poppleton, ${ }^{29}$ X. Portell Bueso, ${ }^{29}$

C. Posch, ${ }^{21}$ G. E. Pospelov, ${ }^{97}$ S. Pospisil, ${ }^{125}$ I. N. Potrap,${ }^{97}$ C. J. Potter,${ }^{147}$ C. T. Potter, ${ }^{112}$ G. Poulard, ${ }^{29}$ J. Poveda, ${ }^{170}$ V. Pozdnyakov, ${ }^{63}$ R. Prabhu, ${ }^{75}$ P. Pralavorio, ${ }^{81}$ A. Pranko, ${ }^{14}$ S. Prasad ${ }^{56}$ R. Pravahan, ${ }^{7}$ S. Prell, ${ }^{62}$ K. Pretzl, ${ }^{16}$ L. Pribyl, ${ }^{29}$ D. Price,${ }^{59}$ J. Price,${ }^{71}$ L. E. Price,${ }^{5}$ M. J. Price,${ }^{29}$ D. Prieur, ${ }^{121}$ M. Primavera, ${ }^{70 a}$ K. Prokofiev,${ }^{106}$

F. Prokoshin, ${ }^{31 b}$ S. Protopopescu, ${ }^{24}$ J. Proudfoot,${ }^{5}$ X. Prudent,${ }^{43}$ M. Przybycien, ${ }^{37}$ H. Przysiezniak, ${ }^{4}$ S. Psoroulas, ${ }^{20}$ E. Ptacek, ${ }^{112}$ E. Pueschel, ${ }^{82}$ J. Purdham, ${ }^{85}$ M. Purohit,${ }^{24, z}$ P. Puzo, ${ }^{113}$ Y. Pylypchenko, ${ }^{61}$ J. Qian ${ }^{85}$ Z. Qian, ${ }^{81}$ Z. Qin, ${ }^{41}$ A. Quadt, ${ }^{53}$ D. R. Quarrie, ${ }^{14}$ W. B. Quayle, ${ }^{170}$ F. Quinonez, ${ }^{31 a}$ M. Raas, ${ }^{102}$ V. Radescu, ${ }^{57 b}$ B. Radics,${ }^{20}$

P. Radloff, ${ }^{112}$ T. Rador, ${ }^{18 \mathrm{a}}$ F. Ragusa, ${ }^{87 a, 87 b}$ G. Rahal, ${ }^{175}$ A. M. Rahimi, ${ }^{107}$ D. Rahm, ${ }^{24}$ S. Rajagopalan, ${ }^{24}$ M. Rammensee, ${ }^{47}$ M. Rammes, ${ }^{139}$ A. S. Randle-Conde, ${ }^{39}$ K. Randrianarivony, ${ }^{28}$ P. N. Ratoff, ${ }^{69}$ F. Rauscher, ${ }^{96}$ T. C. Rave, ${ }^{47}$ M. Raymond, ${ }^{29}$ A. L. Read, ${ }^{115}$ D. M. Rebuzzi, ${ }^{117 a, 117 b}$ A. Redelbach, ${ }^{171}$ G. Redlinger, ${ }^{24}$ R. Reece, ${ }^{118}$ K. Reeves, ${ }^{40}$ A. Reichold, ${ }^{103}$ E. Reinherz-Aronis, ${ }^{151}$ A. Reinsch, ${ }^{112}$ I. Reisinger, ${ }^{42}$ C. Rembser ${ }^{29}$ Z. L. Ren, ${ }^{149}$ A. Renaud, ${ }^{113}$ P. Renkel, ${ }^{39}$ M. Rescigno, ${ }^{130 a}$ S. Resconi, ${ }^{87 a}$ B. Resende, ${ }^{134}$ P. Reznicek, ${ }^{96}$ R. Rezvani, ${ }^{156}$ A. Richards, ${ }^{75}$ R. Richter, ${ }^{97}$ E. Richter-Was, ${ }^{4, c c}$ M. Ridel, ${ }^{76}$ M. Rijpstra, ${ }^{103}$ M. Rijssenbeek, ${ }^{146}$ A. Rimoldi, ${ }^{117 a, 117 b}$ L. Rinaldi, ${ }^{19 a}$ R. R. Rios, ${ }^{39}$ I. Riu, ${ }^{11}$ G. Rivoltella,${ }^{87 a, 87 b}$ F. Rizatdinova, ${ }^{110}$ E. Rizvi, ${ }^{73}$ S. H. Robertson,,${ }^{83, j}$ A. Robichaud-Veronneau, ${ }^{116}$ D. Robinson, ${ }^{27}$ J. E. M. Robinson, ${ }^{75}$ M. Robinson, ${ }^{112}$ A. Robson, ${ }^{52}$ J. G. Rocha de Lima, ${ }^{104}$ C. Roda, ${ }^{120 a, 120 b}$ D. Roda Dos Santos, ${ }^{29}$ D. Rodriguez, ${ }^{160}$ A. Roe,${ }^{53}$ S. Roe,${ }^{29}$ O. Røhne, ${ }^{115}$ V. Rojo, ${ }^{1}$ S. Rolli, ${ }^{159}$ A. Romaniouk, ${ }^{94}$ M. Romano, ${ }^{19 a, 19 b}$ V. M. Romanov, ${ }^{63}$ G. Romeo, ${ }^{26}$ E. Romero Adam, ${ }^{165}$ 
L. Roos, ${ }^{76}$ E. Ros,${ }^{165}$ S. Rosati, ${ }^{130 a}$ K. Rosbach,${ }^{48}$ A. Rose,${ }^{147}$ M. Rose,${ }^{74}$ G. A. Rosenbaum, ${ }^{156}$ E. I. Rosenberg, ${ }^{62}$ P. L. Rosendahl, ${ }^{13}$ O. Rosenthal, ${ }^{139}$ L. Rosselet, ${ }^{48}$ V. Rossetti, ${ }^{11}$ E. Rossi, ${ }^{130 a, 130 b}$ L. P. Rossi, ${ }^{49 a}$ M. Rotaru, ${ }^{25 a}$ I. Roth, ${ }^{169}$ J. Rothberg, ${ }^{136}$ D. Rousseau, ${ }^{113}$ C. R. Royon, ${ }^{134}$ A. Rozanov, ${ }^{81}$ Y. Rozen, ${ }^{150}$ X. Ruan, ${ }^{32 a, d d}$ I. Rubinskiy, ${ }^{41}$

B. Ruckert, ${ }^{96}$ N. Ruckstuhl, ${ }^{103}$ V. I. Rud,${ }^{95}$ C. Rudolph,${ }^{43}$ G. Rudolph,${ }^{60}$ F. Rühr, ${ }^{6}$ F. Ruggieri, ${ }^{132 a, 132 b}$

A. Ruiz-Martinez, ${ }^{62}$ V. Rumiantsev, ${ }^{89, j j}$ L. Rumyantsev, ${ }^{63}$ K. Runge, ${ }^{47}$ Z. Rurikova, ${ }^{47}$ N. A. Rusakovich, ${ }^{63}$

D. R. Rust, ${ }^{59}$ J. P. Rutherfoord, ${ }^{6}$ C. Ruwiedel, ${ }^{14}$ P. Ruzicka, ${ }^{123}$ Y. F. Ryabov, ${ }^{119}$ V. Ryadovikov, ${ }^{126}$ P. Ryan, ${ }^{86}$

M. Rybar, ${ }^{124}$ G. Rybkin, ${ }^{113}$ N. C. Ryder, ${ }^{116}$ S. Rzaeva, ${ }^{10}$ A. F. Saavedra, ${ }^{148}$ I. Sadeh, ${ }^{151}$ H. F-W. Sadrozinski, ${ }^{135}$

R. Sadykov, ${ }^{63}$ F. Safai Tehrani, ${ }^{130 a}$ H. Sakamoto, ${ }^{153}$ G. Salamanna, ${ }^{73}$ A. Salamon, ${ }^{131 a}$ M. Saleem, ${ }^{109}$ D. Salihagic, ${ }^{97}$

A. Salnikov, ${ }^{141}$ J. Salt, ${ }^{165}$ B. M. Salvachua Ferrando, ${ }^{5}$ D. Salvatore, ${ }^{36 a, 36 b}$ F. Salvatore, ${ }^{147}$ A. Salvucci, ${ }^{102}$

A. Salzburger ${ }^{29}$ D. Sampsonidis, ${ }^{152}$ B. H. Samset, ${ }^{115}$ A. Sanchez, ${ }^{100 a, 100 b}$ V. Sanchez Martinez, ${ }^{165}$ H. Sandaker, ${ }^{13}$

H. G. Sander, ${ }^{79}$ M. P. Sanders, ${ }^{96}$ M. Sandhoff, ${ }^{172}$ T. Sandoval, ${ }^{27}$ C. Sandoval, ${ }^{160}$ R. Sandstroem, ${ }^{97}$ S. Sandvoss, ${ }^{172}$

D. P. C. Sankey, ${ }^{127}$ A. Sansoni,${ }^{46}$ C. Santamarina Rios,${ }^{83}$ C. Santoni,${ }^{33}$ R. Santonico, ${ }^{131 a, 131 b}$ H. Santos, ${ }^{122 a}$

J. G. Saraiva, ${ }^{122 \mathrm{a}}$ T. Sarangi, ${ }^{170}$ E. Sarkisyan-Grinbaum, ${ }^{7}$ F. Sarri, ${ }^{120 a, 120 \mathrm{~b}}$ G. Sartisohn, ${ }^{172}$ O. Sasaki, ${ }^{64}$ N. Sasao, ${ }^{66}$

I. Satsounkevitch, ${ }^{88}$ G. Sauvage, ${ }^{4}$ E. Sauvan, ${ }^{4}$ J. B. Sauvan, ${ }^{113}$ P. Savard, ${ }^{156, d}$ V. Savinov, ${ }^{121}$ D. O. Savu, ${ }^{29}$

L. Sawyer, ${ }^{24,1}$ D. H. Saxon, ${ }^{52}$ L. P. Says ${ }^{33}$ C. Sbarra, ${ }^{19 a}$ A. Sbrizzi, ${ }^{19 a, 19 b}$ O. Scallon, ${ }^{91}$ D. A. Scannicchio, ${ }^{161}$

M. Scarcella, ${ }^{148}$ J. Schaarschmidt, ${ }^{113}$ P. Schacht, ${ }^{97}$ U. Schäfer, ${ }^{79}$ S. Schaepe,${ }^{20}$ S. Schaetzel, ${ }^{57 b}$ A. C. Schaffer, ${ }^{113}$

D. Schaile, ${ }^{96}$ R. D. Schamberger, ${ }^{146}$ A. G. Schamov, ${ }^{105}$ V. Scharf,${ }^{57 a}$ V. A. Schegelsky, ${ }^{119}$ D. Scheirich, ${ }^{85}$

M. Schernau, ${ }^{161}$ M. I. Scherzer, ${ }^{34}$ C. Schiavi, ${ }^{49 a, 49 b}$ J. Schieck, ${ }^{96}$ M. Schioppa, ${ }^{36 a, 36 b}$ S. Schlenker, ${ }^{29}$ J. L. Schlereth, ${ }^{5}$

E. Schmidt,${ }^{47}$ K. Schmieden, ${ }^{20}$ C. Schmitt, ${ }^{79}$ S. Schmitt, ${ }^{57 b}$ M. Schmitz, ${ }^{20}$ A. Schöning, ${ }^{57 b}$ M. Schott, ${ }^{29}$

D. Schouten, ${ }^{157 a}$ J. Schovancova, ${ }^{123}$ M. Schram, ${ }^{83}$ C. Schroeder,${ }^{79}$ N. Schroer, ${ }^{57 \mathrm{c}}$ S. Schuh, ${ }^{29}$ G. Schuler, ${ }^{29}$

M. J. Schultens, ${ }^{20}$ J. Schultes, ${ }^{172}$ H.-C. Schultz-Coulon, ${ }^{57 a}$ H. Schulz, ${ }^{15}$ J. W. Schumacher, ${ }^{20}$ M. Schumacher, ${ }^{47}$

B. A. Schumm, ${ }^{135}$ Ph. Schune, ${ }^{134}$ C. Schwanenberger, ${ }^{80}$ A. Schwartzman, ${ }^{141}$ Ph. Schwemling,${ }^{76}$ R. Schwienhorst, ${ }^{86}$

R. Schwierz, ${ }^{43}$ J. Schwindling, ${ }^{134}$ T. Schwindt, ${ }^{20}$ M. Schwoerer,${ }^{4}$ W. G. Scott, ${ }^{127}$ J. Searcy, ${ }^{112}$ G. Sedov, ${ }^{41}$

E. Sedykh, ${ }^{119}$ E. Segura, ${ }^{11}$ S. C. Seidel, ${ }^{101}$ A. Seiden, ${ }^{135}$ F. Seifert, ${ }^{43}$ J. M. Seixas, ${ }^{23 a}$ G. Sekhniaidze,${ }^{100 a}$

K. E. Selbach, ${ }^{45}$ D. M. Seliverstov, ${ }^{119}$ B. Sellden, ${ }^{14 a}$ G. Sellers, ${ }^{71}$ M. Seman, ${ }^{142 b}$ N. Semprini-Cesari, ${ }^{19 a, 19 b}$

C. Serfon, ${ }^{96}$ L. Serin, ${ }^{113}$ L. Serkin, ${ }^{53}$ R. Seuster, ${ }^{97}$ H. Severini, ${ }^{109}$ M. E. Sevior, ${ }^{84}$ A. Sfyrla, ${ }^{29}$ E. Shabalina, ${ }^{53}$

M. Shamim, ${ }^{112}$ L. Y. Shan, ${ }^{32 a}$ J. T. Shank, ${ }^{21}$ Q. T. Shao,${ }^{84}$ M. Shapiro, ${ }^{14}$ P. B. Shatalov, ${ }^{93}$ L. Shaver, ${ }^{6}$ K. Shaw, ${ }^{162 a, 162 c}$ D. Sherman, ${ }^{173}$ P. Sherwood, ${ }^{75}$ A. Shibata, ${ }^{106}$ H. Shichi, ${ }^{99}$ S. Shimizu, ${ }^{29}$ M. Shimojima, ${ }^{98}$ T. Shin, ${ }^{55}$ M. Shiyakova ${ }^{63}$ A. Shmeleva, ${ }^{92}$ M. J. Shochet ${ }^{30}$ D. Short,${ }^{116}$ S. Shrestha,${ }^{62}$ E. Shulga,${ }^{94}$ M. A. Shupe, ${ }^{6}$ P. Sicho, ${ }^{123}$ A. Sidoti, ${ }^{130 a}$

F. Siegert, ${ }^{47}$ Dj. Sijacki, ${ }^{12 a}$ O. Silbert, ${ }^{169}$ J. Silva, ${ }^{122 a, b}$ Y. Silver, ${ }^{151}$ D. Silverstein, ${ }^{141}$ S. B. Silverstein, ${ }^{144 a}$ V. Simak, ${ }^{125}$ O. Simard, ${ }^{134}$ Lj. Simic, ${ }^{12 a}$ S. Simion, ${ }^{113}$ B. Simmons, ${ }^{75}$ M. Simonyan, ${ }^{35}$ P. Sinervo, ${ }^{156}$ N. B. Sinev, ${ }^{112}$ V. Sipica, ${ }^{139}$ G. Siragusa, ${ }^{171}$ A. Sircar, ${ }^{24}$ A. N. Sisakyan, ${ }^{63}$ S. Yu. Sivoklokov, ${ }^{95}$ J. Sjölin, ${ }^{144 a, 144 b}$ T. B. Sjursen, ${ }^{13}$ L. A. Skinnari, ${ }^{14}$ H. P. Skottowe, ${ }^{56}$ K. Skovpen, ${ }^{105}$ P. Skubic, ${ }^{109}$ N. Skvorodnev, ${ }^{22}$ M. Slater, ${ }^{17}$ T. Slavicek, ${ }^{125}$

K. Sliwa, ${ }^{159}$ J. Sloper, ${ }^{29}$ V. Smakhtin, ${ }^{169}$ B. H. Smart, ${ }^{45}$ S. Yu. Smirnov, ${ }^{94}$ Y. Smirnov, ${ }^{94}$ L. N. Smirnova, ${ }^{95}$

O. Smirnova, ${ }^{77}$ B. C. Smith, ${ }^{56}$ D. Smith, ${ }^{141}$ K. M. Smith, ${ }^{52}$ M. Smizanska, ${ }^{69}$ K. Smolek, ${ }^{125}$ A. A. Snesarev, ${ }^{92}$

S. W. Snow, ${ }^{80}$ J. Snow, ${ }^{109}$ J. Snuverink, ${ }^{103}$ S. Snyder, ${ }^{24}$ M. Soares, ${ }^{122 a}$ R. Sobie, ${ }^{167, j}$ J. Sodomka, ${ }^{125}$ A. Soffer, ${ }^{151}$

C. A. Solans, ${ }^{165}$ M. Solar, ${ }^{125}$ J. Solc,${ }^{125}$ E. Soldatov, ${ }^{94}$ U. Soldevila, ${ }^{165}$ E. Solfaroli Camillocci, ${ }^{130 a, 130 \mathrm{~b}}$

A. A. Solodkov, ${ }^{126}$ O. V. Solovyanov, ${ }^{126}$ N. Soni, ${ }^{2}$ V. Sopko, ${ }^{125}$ B. Sopko, ${ }^{125}$ M. Sosebee, ${ }^{7}$ R. Soualah,,${ }^{162 a, 162 c}$

A. Soukharev, ${ }^{105}$ S. Spagnolo, ${ }^{70 a, 70 b}$ F. Spanò ${ }^{74}$ R. Spighi, ${ }^{19 a}$ G. Spigo, ${ }^{29}$ F. Spila, ${ }^{130 a, 130 b}$ R. Spiwoks, ${ }^{29}$

M. Spousta, ${ }^{124}$ T. Spreitzer, ${ }^{156}$ B. Spurlock, ${ }^{7}$ R. D. St. Denis, ${ }^{52}$ J. Stahlman, ${ }^{118}$ R. Stamen, ${ }^{57 a}$ E. Stanecka, ${ }^{38}$

R. W. Stanek, ${ }^{5}$ C. Stanescu, ${ }^{132 a}$ S. Stapnes, ${ }^{115}$ E. A. Starchenko, ${ }^{126}$ J. Stark, ${ }^{54}$ P. Staroba, ${ }^{123}$ P. Starovoitov, ${ }^{89}$

A. Staude, ${ }^{96}$ P. Stavina, ${ }^{142 a}$ G. Stavropoulos, ${ }^{14}$ G. Steele, ${ }^{52}$ P. Steinbach, ${ }^{43}$ P. Steinberg, ${ }^{24}$ I. Stekl, ${ }^{125}$ B. Stelzer, ${ }^{140}$

H. J. Stelzer, ${ }^{86}$ O. Stelzer-Chilton, ${ }^{157 a}$ H. Stenzel, ${ }^{51}$ S. Stern, ${ }^{97}$ K. Stevenson,${ }^{73}$ G. A. Stewart, ${ }^{29}$ J. A. Stillings,${ }^{20}$

M. C. Stockton, ${ }^{83}$ K. Stoerig, ${ }^{47}$ G. Stoicea, ${ }^{25 a}$ S. Stonjek, ${ }^{97}$ P. Strachota, ${ }^{124}$ A. R. Stradling, ${ }^{7}$ A. Straessner, ${ }^{43}$

J. Strandberg, ${ }^{145}$ S. Strandberg, ${ }^{144 a, 144 b}$ A. Strandlie, ${ }^{115}$ M. Strang, ${ }^{107}$ E. Strauss, ${ }^{141}$ M. Strauss, ${ }^{109}$ P. Strizenec, ${ }^{142 b}$

R. Ströhmer, ${ }^{171}$ D. M. Strom, ${ }^{112}$ J. A. Strong, ${ }^{74, j j}$ R. Stroynowski, ${ }^{39}$ J. Strube,${ }^{127}$ B. Stugu, ${ }^{13}$ I. Stumer, ${ }^{24, j j}$

J. Stupak, ${ }^{146}$ P. Sturm, ${ }^{172}$ N. A. Styles, ${ }^{41}$ D. A. Soh, ${ }^{149, u}$ D. Su, ${ }^{141}$ HS. Subramania, ${ }^{2}$ A. Succurro, ${ }^{11}$ Y. Sugaya, ${ }^{114}$

T. Sugimoto, ${ }^{99}$ C. Suhr, ${ }^{104}$ K. Suita, ${ }^{65}$ M. Suk, ${ }^{124}$ V. V. Sulin, ${ }^{92}$ S. Sultansoy, ${ }^{3 d}$ T. Sumida ${ }^{66}$ X. Sun, ${ }^{54}$

J.E. Sundermann, ${ }^{47}$ K. Suruliz, ${ }^{137}$ S. Sushkov, ${ }^{11}$ G. Susinno, ${ }^{36 a, 36 b}$ M. R. Sutton, ${ }^{147}$ Y. Suzuki, ${ }^{64}$ Y. Suzuki, ${ }^{65}$

M. Svatos, ${ }^{123}$ Yu. M. Sviridov, ${ }^{126}$ S. Swedish, ${ }^{166}$ I. Sykora, ${ }^{142 a}$ T. Sykora, ${ }^{124}$ B. Szeless, ${ }^{29}$ J. Sánchez, ${ }^{165}$ D. Ta, ${ }^{103}$

K. Tackmann, ${ }^{41}$ A. Taffard, ${ }^{161}$ R. Tafirout, ${ }^{157 a}$ N. Taiblum,,${ }^{151}$ Y. Takahashi, ${ }^{99}$ H. Takai, ${ }^{24}$ R. Takashima, ${ }^{67}$ 
H. Takeda, ${ }^{65}$ T. Takeshita, ${ }^{138}$ Y. Takubo, ${ }^{64}$ M. Talby ${ }^{81}$ A. Talyshev,,${ }^{105, f}$ M. C. Tamsett,${ }^{24}$ J. Tanaka, ${ }^{153}$ R. Tanaka, ${ }^{113}$ S. Tanaka, ${ }^{129}$ S. Tanaka, ${ }^{64}$ Y. Tanaka ${ }^{98}$ A. J. Tanasijczuk, ${ }^{140}$ K. Tani, ${ }^{65}$ N. Tannoury ${ }^{81}$ G. P. Tappern,${ }^{29}$ S. Tapprogge ${ }^{79}$ D. Tardif, ${ }^{156}$ S. Tarem, ${ }^{150}$ F. Tarrade,${ }^{28}$ G. F. Tartarelli, ${ }^{87 a}$ P. Tas,${ }^{124}$ M. Tasevsky, ${ }^{123}$ E. Tassi, ${ }^{36 a, 36 b}$ M. Tatarkhanov, ${ }^{14}$ Y. Tayalati, ${ }^{133 d}$ C. Taylor, ${ }^{75}$ F. E. Taylor, ${ }^{90}$ G. N. Taylor, ${ }^{84}$ W. Taylor, ${ }^{157 b}$ M. Teinturier, ${ }^{113}$ M. Teixeira Dias Castanheira, ${ }^{73}$ P. Teixeira-Dias, ${ }^{74}$ K. K. Temming, ${ }^{47}$ H. Ten Kate, ${ }^{29}$ P. K. Teng, ${ }^{149}$ S. Terada, ${ }^{64}$ K. Terashi, ${ }^{153}$ J. Terron, ${ }^{78}$ M. Testa, ${ }^{46}$ R. J. Teuscher, ${ }^{156, j}$ J. Thadome, ${ }^{172}$ J. Therhaag, ${ }^{20}$ T. Theveneaux-Pelzer, ${ }^{76}$ M. Thioye, ${ }^{173}$ S. Thoma,${ }^{47}$ J. P. Thomas, ${ }^{17}$ E. N. Thompson, ${ }^{34}$ P. D. Thompson, ${ }^{17}$ P. D. Thompson, ${ }^{156}$ A. S. Thompson, ${ }^{52}$ L. A. Thomsen, ${ }^{35}$ E. Thomson, ${ }^{118}$ M. Thomson, ${ }^{27}$ R. P. Thun ${ }^{85}$ F. Tian, ${ }^{34}$ M. J. Tibbetts, ${ }^{14}$ T. Tic, ${ }^{123}$ V. O. Tikhomirov, ${ }^{92}$ Y. A. Tikhonov, ${ }^{105, f}$ S. Timoshenko, ${ }^{94}$ P. Tipton, ${ }^{173}$ F. J. Tique Aires Viegas,${ }^{29}$ S. Tisserant ${ }^{81}$ B. Toczek,${ }^{37}$ T. Todorov ${ }^{4}$ S. Todorova-Nova, ${ }^{159}$ B. Toggerson, ${ }^{161}$ J. Tojo,${ }^{64}$ S. Tokár, ${ }^{142 a}$ K. Tokunaga,${ }^{65} \mathrm{~K}$. Tokushuku, ${ }^{64} \mathrm{~K}$. Tollefson, ${ }^{86} \mathrm{M}$. Tomoto, ${ }^{99} \mathrm{~L}$. Tompkins,${ }^{30} \mathrm{~K}$. Toms, ${ }^{101} \mathrm{G}$. Tong, ${ }^{32 \mathrm{a}}$ A. Tonoyan,${ }^{13}$ C. Topfel, ${ }^{16}$ N. D. Topilin,${ }^{63}$ I. Torchiani, ${ }^{29}$ E. Torrence, ${ }^{112}$ H. Torres,${ }^{76}$ E. Torró Pastor,${ }^{165}$ J. Toth, ${ }^{81, \text { aa }}$ F. Touchard ${ }^{81}$ D. R. Tovey, ${ }^{137}$ T. Trefzger,${ }^{171}$ L. Tremblet, ${ }^{29}$ A. Tricoli, ${ }^{29}$ I. M. Trigger, ${ }^{157 a}$ S. Trincaz-Duvoid, ${ }^{76}$ T. N. Trinh, ${ }^{76}$ M. F. Tripiana, ${ }^{68}$ W. Trischuk, ${ }^{156}$ A. Trivedi, ${ }^{24, z}$ B. Trocmé,${ }^{54}$ C. Troncon, ${ }^{87 a}$ M. Trottier-McDonald, ${ }^{140}$ M. Trzebinski, ${ }^{38}$ A. Trzupek, ${ }^{38}$ C. Tsarouchas, ${ }^{29}$ J. C-L. Tseng, ${ }^{116}$ M. Tsiakiris,${ }^{103}$ P. V. Tsiareshka, ${ }^{88}$ D. Tsionou, ${ }^{4, e e}$ G. Tsipolitis, ${ }^{9}$ V. Tsiskaridze, ${ }^{47}$ E. G. Tskhadadze, ${ }^{50 a}$ I. I. Tsukerman, ${ }^{93}$ V. Tsulaia,${ }^{14}$ J.-W. Tsung, ${ }^{20}$ S. Tsuno ${ }^{64}$ D. Tsybychev, ${ }^{146}$ A. Tua ${ }^{137}$ A. Tudorache, ${ }^{25 a}$ V. Tudorache,${ }^{25 a}$ J. M. Tuggle,${ }^{30}$ M. Turala ${ }^{38}$ D. Turecek,${ }^{125}$ I. Turk Cakir, ${ }^{3 e}$ E. Turlay, ${ }^{103}$ R. Turra, ${ }^{87 a, 87 b}$ P. M. Tuts, ${ }^{34}$ A. Tykhonov, ${ }^{72}$ M. Tylmad,${ }^{144 a, 144 b}$ M. Tyndel, ${ }^{127}$ G. Tzanakos, ${ }^{8}$ K. Uchida, ${ }^{20}$ I. Ueda, ${ }^{153}$ R. Ueno, ${ }^{28}$ M. Ugland, ${ }^{13}$ M. Uhlenbrock,${ }^{20}$ M. Uhrmacher, ${ }^{53}$ F. Ukegawa, ${ }^{158}$ G. Unal, ${ }^{29}$ D. G. Underwood, ${ }^{5}$ A. Undrus, ${ }^{24}$ G. Unel, ${ }^{161}$ Y. Unno, ${ }^{64}$ D. Urbaniec, ${ }^{34}$ G. Usai, ${ }^{7}$ M. Uslenghi, ${ }^{117 a, 117 b}$ L. Vacavant, ${ }^{81}$ V. Vacek, ${ }^{125}$ B. Vachon, ${ }^{83}$ S. Vahsen, ${ }^{14}$ J. Valenta, ${ }^{123}$ P. Valente, ${ }^{130 a}$ S. Valentinetti, ${ }^{19 a, 19 b}$ S. Valkar, ${ }^{124}$ E. Valladolid Gallego, ${ }^{165}$ S. Vallecorsa, ${ }^{150}$ J. A. Valls Ferrer, ${ }^{165}$ H. van der Graaf, ${ }^{103}$ E. van der Kraaij, ${ }^{103}$

R. Van Der Leeuw, ${ }^{103}$ E. van der Poel, ${ }^{103}$ D. van der Ster, ${ }^{29}$ N. van Eldik, ${ }^{82}$ P. van Gemmeren, ${ }^{5}$ Z. van Kesteren, ${ }^{103}$ I. van Vulpen, ${ }^{103}$ M. Vanadia, ${ }^{97}$ W. Vandelli, ${ }^{29}$ G. Vandoni, ${ }^{29}$ A. Vaniachine, ${ }^{5}$ P. Vankov,${ }^{41}$ F. Vannucci, ${ }^{76}$

F. Varela Rodriguez ${ }^{29}$ R. Vari, ${ }^{130 a}$ E. W. Varnes, ${ }^{6}$ D. Varouchas, ${ }^{14}$ A. Vartapetian, ${ }^{7}$ K. E. Varvell,,${ }^{148}$

V. I. Vassilakopoulos ${ }^{55}$ F. Vazeille, ${ }^{33}$ G. Vegni, ${ }^{87 a, 87 b}$ J. J. Veillet, ${ }^{113}$ C. Vellidis, ${ }^{8}$ F. Veloso, ${ }^{122 a}$ R. Veness, ${ }^{29}$ S. Veneziano, ${ }^{130 a}$ A. Ventura, ${ }^{70 a, 70 b}$ D. Ventura, ${ }^{136}$ M. Venturi, ${ }^{47}$ N. Venturi, ${ }^{156}$ V. Vercesi, ${ }^{117 a}$ M. Verducci, ${ }^{136}$ W. Verkerke, ${ }^{103}$ J. C. Vermeulen, ${ }^{103}$ A. Vest ${ }^{43}$ M.C. Vetterli, ${ }^{140, d}$ I. Vichou, ${ }^{163}$ T. Vickey, ${ }^{143 b, f f}$

O. E. Vickey Boeriu, ${ }^{143 b}$ G. H. A. Viehhauser, ${ }^{116}$ S. Viel,,${ }^{166}$ M. Villa, ${ }^{19 a, 19 b}$ M. Villaplana Perez, ${ }^{165}$ E. Vilucchi, ${ }^{46}$ M. G. Vincter, ${ }^{28}$ E. Vinek, ${ }^{29}$ V. B. Vinogradov ${ }^{63}$ M. Virchaux, ${ }^{134, j j}$ J. Virzi, ${ }^{14}$ O. Vitells ${ }^{169}$ M. Viti, ${ }^{41}$ I. Vivarelli, ${ }^{47}$ F. Vives Vaque, ${ }^{2}$ S. Vlachos, ${ }^{9}$ D. Vladoiu, ${ }^{96}$ M. Vlasak, ${ }^{125}$ N. Vlasov, ${ }^{20}$ A. Vogel,${ }^{20}$ P. Vokac, ${ }^{125}$ G. Volpi,${ }^{46}$ M. Volpi,${ }^{84}$ G. Volpini, ${ }^{87 a}$ H. von der Schmitt ${ }^{97}$ J. von Loeben, ${ }^{97}$ H. von Radziewski, ${ }^{47}$ E. von Toerne,${ }^{20}$ V. Vorobel, ${ }^{124}$ A. P. Vorobiev, ${ }^{126}$ V. Vorwerk, ${ }^{11}$ M. Vos, ${ }^{165}$ R. Voss, ${ }^{29}$ T. T. Voss, ${ }^{172}$ J. H. Vossebeld, ${ }^{71}$ N. Vranjes,${ }^{134}$ M. Vranjes Milosavljevic, ${ }^{103} \mathrm{~V}$. Vrba, ${ }^{123}$ M. Vreeswijk, ${ }^{103}$ T. Vu Anh, ${ }^{47}$ R. Vuillermet, ${ }^{29}$ I. Vukotic, ${ }^{113}$ W. Wagner, ${ }^{172} \mathrm{P}$. Wagner, ${ }^{118} \mathrm{H}$. Wahlen, ${ }^{172} \mathrm{~J}$. Wakabayashi, ${ }^{99} \mathrm{~J}$. Walbersloh, ${ }^{42} \mathrm{~S}$. Walch, ${ }^{85} \mathrm{~J}$. Walder, ${ }^{69} \mathrm{R}$. Walker, ${ }^{96}$ W. Walkowiak, ${ }^{139}$ R. Wall, ${ }^{173}$ P. Waller, ${ }^{71}$ C. Wang, ${ }^{44}$ H. Wang,,${ }^{170}$ H. Wang, ${ }^{32 b, g g}$ J. Wang, ${ }^{149}$ J. Wang, ${ }^{54}$ J. C. Wang, ${ }^{136}$ R. Wang, ${ }^{101}$ S. M. Wang, ${ }^{149}$ A. Warburton, ${ }^{83}$ C. P. Ward, ${ }^{27}$ M. Warsinsky,${ }^{47}$ P. M. Watkins, ${ }^{17}$ A. T. Watson, ${ }^{17}$ I. J. Watson, ${ }^{148}$ M. F. Watson, ${ }^{17}$ G. Watts, ${ }^{136}$ S. Watts, ${ }^{80}$ A. T. Waugh, ${ }^{148}$ B. M. Waugh, ${ }^{75}$ M. Weber, ${ }^{127}$ M. S. Weber, ${ }^{16}$ P. Weber, ${ }^{53}$ A. R. Weidberg,,${ }^{116}$ P. Weigell, ${ }^{97}$ J. Weingarten, ${ }^{53}$ C. Weiser, ${ }^{47}$ H. Wellenstein, ${ }^{22}$ P. S. Wells,${ }^{29}$ M. Wen, ${ }^{46}$ T. Wenaus, ${ }^{24}$ D. Wendland, ${ }^{15}$ S. Wendler, ${ }^{121}$ Z. Weng,,${ }^{149, \mathrm{u}}$ T. Wengler,${ }^{29}$ S. Wenig, ${ }^{29} \mathrm{~N}$. Wermes,${ }^{20} \mathrm{M}$. Werner, ${ }^{47}$ P. Werner, ${ }^{29} \mathrm{M}$. Werth, ${ }^{161} \mathrm{M}$. Wessels, ${ }^{57 \mathrm{a}} \mathrm{C}$. Weydert, ${ }^{54} \mathrm{~K}$. Whalen, ${ }^{28}$ S. J. Wheeler-Ellis, ${ }^{161}$ S. P. Whitaker, ${ }^{21}$ A. White, ${ }^{7}$ M. J. White, ${ }^{84}$ S. R. Whitehead, ${ }^{116}$ D. Whiteson, ${ }^{161}$ D. Whittington, ${ }^{59}$ F. Wicek, ${ }^{113}$ D. Wicke, ${ }^{172}$ F. J. Wickens, ${ }^{127}$ W. Wiedenmann, ${ }^{170}$ M. Wielers, ${ }^{127}$ P. Wienemann, ${ }^{20}$ C. Wiglesworth, ${ }^{73}$ L. A. M. Wiik-Fuchs,${ }^{47}$ P. A. Wijeratne, ${ }^{75}$ A. Wildauer, ${ }^{165}$ M. A. Wildt,,${ }^{41, q}$ I. Wilhelm, ${ }^{124}$ H. G. Wilkens, ${ }^{29}$ J. Z. Will,${ }^{96}$ E. Williams,${ }^{34}$ H. H. Williams, ${ }^{118}$ W. Willis, ${ }^{34}$ S. Willocq, ${ }^{82}$ J. A. Wilson, ${ }^{17}$ M. G. Wilson, ${ }^{141}$ A. Wilson, ${ }^{85}$ I. Wingerter-Seez ${ }^{4}$ S. Winkelmann,${ }^{47}$ F. Winklmeier, ${ }^{29}$ M. Wittgen, ${ }^{141}$ M. W. Wolter, ${ }^{38}$ H. Wolters, ${ }^{122 a, h}$ W. C. Wong, ${ }^{40}$ G. Wooden, ${ }^{85}$ B. K. Wosiek,${ }^{38}$ J. Wotschack, ${ }^{29}$ M. J. Woudstra, ${ }^{82}$ K. W. Wozniak, ${ }^{38}$ K. Wraight, ${ }^{52}$ C. Wright, ${ }^{52}$ M. Wright, ${ }^{52}$ B. Wrona, ${ }^{71}$ S. L. Wu, ${ }^{170}$ X. Wu, ${ }^{48}$ Y. Wu, ${ }^{32 b, h h}$ E. Wulf, ${ }^{34}$ R. Wunstorf, ${ }^{42}$ B. M. Wynne, ${ }^{45}$ S. Xella,${ }^{35}$ M. Xiao, ${ }^{134}$ S. Xie, ${ }^{47}$ Y. Xie, ${ }^{32 a}$ C. Xu, ${ }^{32 b, w}$ D. Xu,${ }^{137}$ G. Xu, ${ }^{32 a}$ B. Yabsley, ${ }^{148}$ S. Yacoob, ${ }^{143 b}$ M. Yamada, ${ }^{64}$ H. Yamaguchi, ${ }^{153}$ A. Yamamoto,${ }^{64}$ K. Yamamoto, ${ }^{62}$ S. Yamamoto, ${ }^{153}$ T. Yamamura, ${ }^{153}$ T. Yamanaka, ${ }^{153}$ J. Yamaoka, ${ }^{44}$ T. Yamazaki, ${ }^{153}$ Y. Yamazaki, ${ }^{65}$ Z. Yan, ${ }^{21}$ H. Yang,${ }^{85}$ U. K. Yang ${ }^{80}$ 
Y. Yang, ${ }^{59}$ Y. Yang, ${ }^{32 a}$ Z. Yang, ${ }^{144 a, 144 b}$ S. Yanush, ${ }^{89}$ Y. Yao, ${ }^{14}$ Y. Yasu, ${ }^{64}$ G. V. Ybeles Smit, ${ }^{128}$ J. Ye, ${ }^{39}$ S. Ye, ${ }^{24}$ M. Yilmaz, ${ }^{3 \mathrm{c}} \mathrm{R}$. Yoosoofmiya, ${ }^{121} \mathrm{~K}$. Yorita, ${ }^{168}$ R. Yoshida, ${ }^{5}$ C. Young, ${ }^{141}$ S. Youssef, ${ }^{21}$ D. Yu, ${ }^{24} \mathrm{~J} . \mathrm{Yu},{ }^{7} \mathrm{~J} . \mathrm{Yu},{ }^{110}$ L. Yuan, ${ }^{32 a, i i}$ A. Yurkewicz, ${ }^{104}$ B. Zabinski, ${ }^{38}$ V. G. Zaets, ${ }^{126}$ R. Zaidan, ${ }^{61}$ A. M. Zaitsev, ${ }^{126}$ Z. Zajacova, ${ }^{29}$ L. Zanello, ${ }^{130 a, 130 b}$ P. Zarzhitsky, ${ }^{39}$ A. Zaytsev, ${ }^{105}$ C. Zeitnitz, ${ }^{172}$ M. Zeller, ${ }^{173}$ M. Zeman, ${ }^{123}$ A. Zemla ${ }^{38}$ C. Zendler, ${ }^{20}$ O. Zenin, ${ }^{126}$ T. Ženišs, ${ }^{142 a}$ Z. Zinonos, ${ }^{120 a, 120 b}$ S. Zenz, ${ }^{14}$ D. Zerwas, ${ }^{113}$ G. Zevi della Porta, ${ }^{56}$ Z. Zhan, ${ }^{32 \mathrm{~d}}$ D. Zhang, ${ }^{32 \mathrm{~b}, \mathrm{gg}}$ H. Zhang, ${ }^{86}$ J. Zhang, ${ }^{5}$ X. Zhang, ${ }^{32 \mathrm{~d}}$ Z. Zhang, ${ }^{113}$ L. Zhao, ${ }^{106}$ T. Zhao, ${ }^{136}$ Z. Zhao, ${ }^{32 \mathrm{~b}}$ A. Zhemchugov, ${ }^{63}$ S. Zheng, ${ }^{32 a}$ J. Zhong, ${ }^{116}$ B. Zhou, ${ }^{85}$ N. Zhou, ${ }^{161}$ Y. Zhou, ${ }^{149}$ C. G. Zhu, ${ }^{32 d}$ H. Zhu, ${ }^{41}$ J. Zhu, ${ }^{85}$ Y. Zhu, ${ }^{32 b}$ X. Zhuang, ${ }^{96}$ V. Zhuravlov ${ }^{97}$ D. Zieminska, ${ }^{59}$ R. Zimmermann, ${ }^{20}$ S. Zimmermann, ${ }^{20}$ S. Zimmermann, ${ }^{47}$ M. Ziolkowski, ${ }^{139}$ R. Zitoun, ${ }^{4}$ L. Živković, ${ }^{34}$ V. V. Zmouchko, ${ }^{126, j j}$ G. Zobernig, ${ }^{170}$ A. Zoccoli, ${ }^{19 a, 19 b}$ Y. Zolnierowski, ${ }^{4}$ A. Zsenei, ${ }^{29}$ M. zur Nedden, ${ }^{15}$ V. Zutshi, ${ }^{104}$ and L. Zwalinski ${ }^{29}$

(ATLAS Collaboration)

\footnotetext{
${ }^{1}$ University at Albany, Albany, NY, United States of America

${ }^{2}$ Department of Physics, University of Alberta, Edmonton AB, Canada

${ }^{3 a}$ Department of Physics, Ankara University, Ankara, Turkey

${ }^{3 \mathrm{~b}}$ Department of Physics, Dumlupinar University, Kutahya, Turkey

${ }^{3 \mathrm{c}}$ Department of Physics, Gazi University, Ankara, Turkey

${ }^{3 \mathrm{~d}}$ Division of Physics, TOBB University of Economics and Technology, Ankara, Turkey

${ }^{3 \mathrm{e}}$ Turkish Atomic Energy Authority, Ankara, Turkey

${ }^{4}$ LAPP, CNRS/IN2P3 and Université de Savoie, Annecy-le-Vieux, France

${ }^{5}$ High Energy Physics Division, Argonne National Laboratory, Argonne, IL, United States of America

${ }^{6}$ Department of Physics, University of Arizona, Tucson, AZ, United States of America

${ }^{7}$ Department of Physics, The University of Texas at Arlington, Arlington, TX, United States of America

${ }^{8}$ Physics Department, University of Athens, Athens, Greece

${ }^{9}$ Physics Department, National Technical University of Athens, Zografou, Greece

${ }^{10}$ Institute of Physics, Azerbaijan Academy of Sciences, Baku, Azerbaijan

${ }^{11}$ Institut de Física d'Altes Energies and Departament de Física de la Universitat Autònoma de Barcelona and ICREA, Barcelona, Spain

${ }^{12 \mathrm{a}}$ Institute of Physics, University of Belgrade, Belgrade, Serbia

${ }^{12 \mathrm{~b}}$ Vinca Institute of Nuclear Sciences, University of Belgrade, Belgrade, Serbia

${ }^{13}$ Department for Physics and Technology, University of Bergen, Bergen, Norway

${ }^{14}$ Physics Division, Lawrence Berkeley National Laboratory and University of California, Berkeley, CA, United States of America

${ }^{15}$ Department of Physics, Humboldt University, Berlin, Germany

${ }^{16}$ Albert Einstein Center for Fundamental Physics and Laboratory for High Energy Physics, University of Bern, Bern, Switzerland

${ }^{17}$ School of Physics and Astronomy, University of Birmingham, Birmingham, United Kingdom

${ }^{18 \mathrm{a}}$ Department of Physics, Bogazici University, Istanbul, Turkey

${ }^{18 \mathrm{~b}}$ Division of Physics, Dogus University, Istanbul, Turkey

${ }^{18 \mathrm{c}}$ Department of Physics Engineering, Gaziantep University, Gaziantep, Turkey

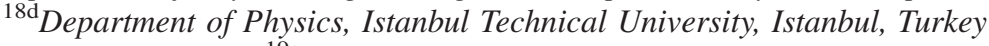

${ }^{19 a}$ INFN Sezione di Bologna, Italy

${ }^{19 b}$ Dipartimento di Fisica, Università di Bologna, Bologna, Italy

${ }^{20}$ Physikalisches Institut, University of Bonn, Bonn, Germany

${ }^{21}$ Department of Physics, Boston University, Boston, MA, United States of America

${ }^{22}$ Department of Physics, Brandeis University, Waltham, MA, United States of America

${ }^{23 a}$ Universidade Federal do Rio De Janeiro COPPE/EE/IF, Rio de Janeiro, Brazil

${ }^{23 \mathrm{~b}}$ Federal University of Juiz de Fora (UFJF), Juiz de Fora, Brazil

${ }^{23 \mathrm{c}}$ Federal University of Sao Joao del Rei (UFSJ), Sao Joao del Rei, Brazil

${ }^{23 \mathrm{~d}}$ Instituto de Fisica, Universidade de Sao Paulo, Sao Paulo, Brazil

${ }^{24}$ Physics Department, Brookhaven National Laboratory, Upton, NY, United States of America

${ }^{25 a}$ National Institute of Physics and Nuclear Engineering, Bucharest, Romania

${ }^{25 \mathrm{~b}}$ University Politehnica Bucharest, Bucharest, Romania

${ }^{25 c}$ West University in Timisoara, Timisoara, Romania

${ }^{26}$ Departamento de Física, Universidad de Buenos Aires, Buenos Aires, Argentina

${ }^{27}$ Cavendish Laboratory, University of Cambridge, Cambridge, United Kingdom

${ }^{28}$ Department of Physics, Carleton University, Ottawa ON, Canada

${ }^{29}$ CERN, Geneva, Switzerland

${ }^{30}$ Enrico Fermi Institute, University of Chicago, Chicago, IL, United States of America
} 
${ }^{31 a}$ Departamento de Fisica, Pontificia Universidad Católica de Chile, Santiago, Chile

${ }^{31 \mathrm{~b}}$ Departamento de Física, Universidad Técnica Federico Santa María, Valparaíso, Chile

${ }^{32 \mathrm{a}}$ Institute of High Energy Physics, Chinese Academy of Sciences, Beijing, China

${ }^{32 \mathrm{~b}}$ Department of Modern Physics, University of Science and Technology of China, Anhui, China

${ }^{32 \mathrm{c}}$ Department of Physics, Nanjing University, Jiangsu, China

${ }^{32 \mathrm{~d}}$ School of Physics, Shandong University, Shandong, China

${ }^{33}$ Laboratoire de Physique Corpusculaire, Clermont Université and Université Blaise Pascal and CNRS/IN2P3, Aubiere Cedex, France

${ }^{34}$ Nevis Laboratory, Columbia University, Irvington, NY, United States of America

${ }^{35}$ Niels Bohr Institute, University of Copenhagen, Kobenhavn, Denmark

${ }^{36 a}$ INFN Gruppo Collegato di Cosenza, Italy

${ }^{36 \mathrm{~b}}$ Dipartimento di Fisica, Università della Calabria, Arcavata di Rende, Italy

${ }^{37}$ AGH University of Science and Technology, Faculty of Physics and Applied Computer Science, Krakow, Poland

${ }^{38}$ The Henryk Niewodniczanski Institute of Nuclear Physics, Polish Academy of Sciences, Krakow, Poland

${ }^{39}$ Physics Department, Southern Methodist University, Dallas, TX, United States of America

${ }^{40}$ Physics Department, University of Texas at Dallas, Richardson, TX, United States of America

${ }^{41}$ DESY, Hamburg and Zeuthen, Germany

${ }^{42}$ Institut für Experimentelle Physik IV, Technische Universität Dortmund, Dortmund, Germany

${ }^{43}$ Institut für Kern- und Teilchenphysik, Technical University Dresden, Dresden, Germany

${ }^{44}$ Department of Physics, Duke University, Durham, NC, United States of America

${ }^{45}$ SUPA-School of Physics and Astronomy, University of Edinburgh, Edinburgh, United Kingdom

${ }^{46}$ INFN Laboratori Nazionali di Frascati, Frascati, Italy

${ }^{47}$ Fakultät für Mathematik und Physik, Albert-Ludwigs-Universität, Freiburg i. Br., Germany

${ }^{48}$ Section de Physique, Université de Genève, Geneva, Switzerland

${ }^{49}$ INFN Sezione di Genova, Italy

${ }^{49 b}$ Dipartimento di Fisica, Università di Genova, Genova, Italy

${ }^{50 a}$ E. Andronikashvili Institute of Physics, Tbilisi State University, Tbilisi, Georgia

${ }^{50 \mathrm{~b}}$ High Energy Physics Institute, Tbilisi State University, Tbilisi, Georgia

${ }^{51}$ II Physikalisches Institut, Justus-Liebig-Universität Giessen, Giessen, Germany

${ }^{52}$ SUPA - School of Physics and Astronomy, University of Glasgow, Glasgow, United Kingdom

${ }^{53}$ II Physikalisches Institut, Georg-August-Universität, Göttingen, Germany

${ }^{54}$ Laboratoire de Physique Subatomique et de Cosmologie, Université Joseph Fourier and CNRS/IN2P3 and Institut National Polytechnique de Grenoble, Grenoble, France

${ }^{55}$ Department of Physics, Hampton University, Hampton, VA, United States of America

${ }^{56}$ Laboratory for Particle Physics and Cosmology, Harvard University, Cambridge, MA, United States of America

${ }^{57 a}$ Kirchhoff-Institut für Physik, Ruprecht-Karls-Universität Heidelberg, Heidelberg, Germany

${ }^{57 b}$ Physikalisches Institut, Ruprecht-Karls-Universität Heidelberg, Heidelberg, Germany

${ }^{57 \mathrm{c}}$ ZITI Institut für technische Informatik, Ruprecht-Karls-Universität Heidelberg, Mannheim, Germany

${ }^{58}$ Faculty of Applied Information Science, Hiroshima Institute of Technology, Hiroshima, Japan

${ }^{59}$ Department of Physics, Indiana University, Bloomington, IN, United States of America

${ }^{60}$ Institut für Astro- und Teilchenphysik, Leopold-Franzens-Universität, Innsbruck, Austria

${ }^{61}$ University of Iowa, Iowa City, IA, United States of America

${ }^{62}$ Department of Physics and Astronomy, Iowa State University, Ames, IA, United States of America

${ }^{63}$ Joint Institute for Nuclear Research, JINR Dubna, Dubna, Russia

${ }^{64}$ KEK, High Energy Accelerator Research Organization, Tsukuba, Japan

${ }^{65}$ Graduate School of Science, Kobe University, Kobe, Japan

${ }^{66}$ Faculty of Science, Kyoto University, Kyoto, Japan

${ }^{67}$ Kyoto University of Education, Kyoto, Japan

${ }^{68}$ Instituto de Física La Plata, Universidad Nacional de La Plata and CONICET, La Plata, Argentina

${ }^{69}$ Physics Department, Lancaster University, Lancaster, United Kingdom

${ }^{70 \mathrm{a}}$ INFN Sezione di Lecce, Italy

${ }^{70 \mathrm{~b}}$ Dipartimento di Fisica, Università del Salento, Lecce, Italy

${ }^{71}$ Oliver Lodge Laboratory, University of Liverpool, Liverpool, United Kingdom

${ }^{72}$ Department of Physics, Jožef Stefan Institute and University of Ljubljana, Ljubljana, Slovenia

${ }^{73}$ School of Physics and Astronomy, Queen Mary University of London, London, United Kingdom

${ }^{74}$ Department of Physics, Royal Holloway University of London, Surrey, United Kingdom

${ }^{75}$ Department of Physics and Astronomy, University College London, London, United Kingdom

${ }^{76}$ Laboratoire de Physique Nucléaire et de Hautes Energies, UPMC and Université Paris-Diderot and CNRS/IN2P3, Paris, France

${ }^{77}$ Fysiska institutionen, Lunds universitet, Lund, Sweden

${ }^{78}$ Departamento de Fisica Teorica C-15, Universidad Autonoma de Madrid, Madrid, Spain

${ }^{79}$ Institut für Physik, Universität Mainz, Mainz, Germany 
${ }^{80}$ School of Physics and Astronomy, University of Manchester, Manchester, United Kingdom

${ }^{81} C P P M$, Aix-Marseille Université and CNRS/IN2P3, Marseille, France

${ }^{82}$ Department of Physics, University of Massachusetts, Amherst, MA, United States of America

${ }^{83}$ Department of Physics, McGill University, Montreal QC, Canada

${ }^{84}$ School of Physics, University of Melbourne, Victoria, Australia

${ }^{85}$ Department of Physics, The University of Michigan, Ann Arbor, MI, United States of America

${ }^{86}$ Department of Physics and Astronomy, Michigan State University, East Lansing, MI, United States of America

${ }^{87}$ INFN Sezione di Milano, Italy

${ }^{87 \mathrm{~b}}$ Dipartimento di Fisica, Università di Milano, Milano, Italy

${ }^{88}$ B. I. Stepanov Institute of Physics, National Academy of Sciences of Belarus, Minsk, Republic of Belarus

${ }^{89}$ National Scientific and Educational Centre for Particle and High Energy Physics, Minsk, Republic of Belarus

${ }^{90}$ Department of Physics, Massachusetts Institute of Technology, Cambridge, MA, United States of America

${ }^{91}$ Group of Particle Physics, University of Montreal, Montreal QC, Canada

${ }^{92}$ P. N. Lebedev Institute of Physics, Academy of Sciences, Moscow, Russia

${ }^{93}$ Institute for Theoretical and Experimental Physics (ITEP), Moscow, Russia

${ }^{94}$ Moscow Engineering and Physics Institute (MEPhI), Moscow, Russia

${ }^{95}$ Skobeltsyn Institute of Nuclear Physics, Lomonosov Moscow State University, Moscow, Russia

${ }^{96}$ Fakultät für Physik, Ludwig-Maximilians-Universität München, München, Germany

${ }^{97}$ Max-Planck-Institut für Physik (Werner-Heisenberg-Institut), München, Germany

${ }^{98}$ Nagasaki Institute of Applied Science, Nagasaki, Japan

${ }^{99}$ Graduate School of Science, Nagoya University, Nagoya, Japan

${ }^{100 a}$ INFN Sezione di Napoli, Italy

${ }^{100 \mathrm{~b}}$ Dipartimento di Scienze Fisiche, Università di Napoli, Napoli, Italy

${ }^{101}$ Department of Physics and Astronomy, University of New Mexico, Albuquerque, NM, United States of America

${ }^{102}$ Institute for Mathematics, Astrophysics and Particle Physics, Radboud University Nijmegen/Nikhef, Nijmegen, Netherlands

${ }^{103}$ Nikhef National Institute for Subatomic Physics and University of Amsterdam, Amsterdam, Netherlands

${ }^{104}$ Department of Physics, Northern Illinois University, DeKalb, IL, United States of America

${ }^{105}$ Budker Institute of Nuclear Physics, SB RAS, Novosibirsk, Russia

${ }^{106}$ Department of Physics, New York University, New York, NY, United States of America

${ }^{107}$ Ohio State University, Columbus, OH, United States of America

${ }^{108}$ Faculty of Science, Okayama University, Okayama, Japan

${ }^{109}$ Homer L. Dodge Department of Physics and Astronomy, University of Oklahoma, Norman, OK, United States of America

${ }^{110}$ Department of Physics, Oklahoma State University, Stillwater, OK, United States of America

${ }^{111}$ Palacký University, RCPTM, Olomouc, Czech Republic

${ }^{112}$ Center for High Energy Physics, University of Oregon, Eugene, OR, United States of America

${ }^{113}$ LAL, Univ. Paris-Sud and CNRS/IN2P3, Orsay, France

${ }^{114}$ Graduate School of Science, Osaka University, Osaka, Japan

${ }^{115}$ Department of Physics, University of Oslo, Oslo, Norway

${ }^{116}$ Department of Physics, Oxford University, Oxford, United Kingdom

${ }^{117 a}$ INFN Sezione di Pavia, Italy

${ }^{117 \mathrm{~b}}$ Dipartimento di Fisica, Università di Pavia, Pavia, Italy

${ }^{118}$ Department of Physics, University of Pennsylvania, Philadelphia, PA, United States of America

${ }^{119}$ Petersburg Nuclear Physics Institute, Gatchina, Russia

${ }^{120 a}$ INFN Sezione di Pisa, Italy

${ }^{120 b}$ Dipartimento di Fisica E. Fermi, Università di Pisa, Pisa, Italy

${ }^{121}$ Department of Physics and Astronomy, University of Pittsburgh, Pittsburgh, PA, United States of America

${ }^{122 a}$ Laboratorio de Instrumentacao e Fisica Experimental de Particulas-LIP, Lisboa, Portugal

${ }^{122 \mathrm{~b}}$ Departamento de Fisica Teorica y del Cosmos and CAFPE, Universidad de Granada, Granada, Spain

${ }^{123}$ Institute of Physics, Academy of Sciences of the Czech Republic, Praha, Czech Republic

${ }^{124}$ Faculty of Mathematics and Physics, Charles University in Prague, Praha, Czech Republic

${ }^{125}$ Czech Technical University in Prague, Praha, Czech Republic

${ }^{126}$ State Research Center Institute for High Energy Physics, Protvino, Russia

${ }^{127}$ Particle Physics Department, Rutherford Appleton Laboratory, Didcot, United Kingdom

${ }^{128}$ Physics Department, University of Regina, Regina SK, Canada

${ }^{129}$ Ritsumeikan University, Kusatsu, Shiga, Japan

${ }^{130 a}$ INFN Sezione di Roma I, Italy

${ }^{130 \mathrm{~b}}$ Dipartimento di Fisica, Università La Sapienza, Roma, Italy

${ }^{131}$ INFN Sezione di Roma Tor Vergata, Italy

${ }^{131 \mathrm{~b}}$ Dipartimento di Fisica, Università di Roma Tor Vergata, Roma, Italy

${ }^{132 a}$ INFN Sezione di Roma Tre, Italy

${ }^{132 b}$ Dipartimento di Fisica, Università Roma Tre, Roma, Italy 
${ }^{133 a}$ Faculté des Sciences Ain Chock, Réseau Universitaire de Physique des Hautes Energies-Université Hassan II, Casablanca, Morocco

${ }^{133 b}$ Centre National de l'Energie des Sciences Techniques Nucleaires, Rabat, Morocco

${ }^{133 c}$ Faculté des Sciences Semlalia, Université Cadi Ayyad, LPHEA-Marrakech, Morocco

${ }^{133 \mathrm{~d}}$ Faculté des Sciences, Université Mohamed Premier and LPTPM, Oujda, Morocco

${ }^{133}$ Faculté des Sciences, Université Mohammed V-Agdal, Rabat, Morocco

${ }^{134}$ DSM/IRFU (Institut de Recherches sur les Lois Fondamentales de l'Univers), CEA Saclay

(Commissariat a l'Energie Atomique), Gif-sur-Yvette, France

${ }^{135}$ Santa Cruz Institute for Particle Physics, University of California Santa Cruz, Santa Cruz, CA, United States of America

${ }^{136}$ Department of Physics, University of Washington, Seattle, WA, United States of America

${ }^{137}$ Department of Physics and Astronomy, University of Sheffield, Sheffield, United Kingdom

${ }^{138}$ Department of Physics, Shinshu University, Nagano, Japan

${ }^{139}$ Fachbereich Physik, Universität Siegen, Siegen, Germany

${ }^{140}$ Department of Physics, Simon Fraser University, Burnaby BC, Canada

${ }^{141}$ SLAC National Accelerator Laboratory, Stanford, CA, United States of America

${ }^{142 a}$ Faculty of Mathematics, Physics \& Informatics, Comenius University, Bratislava, Slovak Republic

${ }^{142 \mathrm{~b}}$ Department of Subnuclear Physics, Institute of Experimental Physics of the Slovak Academy of Sciences, Kosice, Slovak Republic

${ }^{143 a}$ Department of Physics, University of Johannesburg, Johannesburg, South Africa

${ }^{143 \mathrm{~b}}$ School of Physics, University of the Witwatersrand, Johannesburg, South Africa

${ }^{144 a}$ Department of Physics, Stockholm University, Sweden

${ }^{144 \mathrm{~b}}$ The Oskar Klein Centre, Stockholm, Sweden

${ }^{145}$ Physics Department, Royal Institute of Technology, Stockholm, Sweden

${ }^{146}$ Departments of Physics \& Astronomy and Chemistry, Stony Brook University, Stony Brook, NY, United States of America

${ }^{147}$ Department of Physics and Astronomy, University of Sussex, Brighton, United Kingdom

${ }^{148}$ School of Physics, University of Sydney, Sydney, Australia

${ }^{149}$ Institute of Physics, Academia Sinica, Taipei, Taiwan

${ }^{150}$ Department of Physics, Technion: Israel Inst. of Technology, Haifa, Israel

${ }^{151}$ Raymond and Beverly Sackler School of Physics and Astronomy, Tel Aviv University, Tel Aviv, Israel

${ }^{152}$ Department of Physics, Aristotle University of Thessaloniki, Thessaloniki, Greece

${ }^{153}$ International Center for Elementary Particle Physics and Department of Physics, The University of Tokyo, Tokyo, Japan

${ }^{154}$ Graduate School of Science and Technology, Tokyo Metropolitan University, Tokyo, Japan

${ }^{155}$ Department of Physics, Tokyo Institute of Technology, Tokyo, Japan

${ }^{156}$ Department of Physics, University of Toronto, Toronto ON, Canada

${ }^{157 a}$ TRIUMF, Vancouver BC, Canada

${ }^{157 b}$ Department of Physics and Astronomy, York University, Toronto ON, Canada

${ }^{158}$ Institute of Pure and Applied Sciences, University of Tsukuba, 1-1-1 Tennodai,Tsukuba, Ibaraki 305-8571, Japan

${ }^{159}$ Science and Technology Center, Tufts University, Medford, MA, United States of America

${ }^{160}$ Centro de Investigaciones, Universidad Antonio Narino, Bogota, Colombia

${ }^{161}$ Department of Physics and Astronomy, University of California Irvine, Irvine, CA, United States of America

${ }^{162 a}$ INFN Gruppo Collegato di Udine, Italy

${ }^{162 \mathrm{~b}}$ ICTP, Trieste, Italy

${ }^{162 \mathrm{c}}$ Dipartimento di Chimica, Fisica e Ambiente, Università di Udine, Udine, Italy

${ }^{163}$ Department of Physics, University of Illinois, Urbana, IL, United States of America

${ }^{164}$ Department of Physics and Astronomy, University of Uppsala, Uppsala, Sweden

${ }^{165}$ Instituto de Física Corpuscular (IFIC) and Departamento de Física Atómica, Molecular y Nuclear and Departamento de Ingeniería Electrónica and Instituto de Microelectrónica de Barcelona (IMB-CNM),

University of Valencia and CSIC, Valencia, Spain

${ }^{166}$ Department of Physics, University of British Columbia, Vancouver BC, Canada

${ }^{167}$ Department of Physics and Astronomy, University of Victoria, Victoria BC, Canada

${ }^{168}$ Waseda University, Tokyo, Japan

${ }^{169}$ Department of Particle Physics, The Weizmann Institute of Science, Rehovot, Israel

${ }^{170}$ Department of Physics, University of Wisconsin, Madison, WI, United States of America

${ }^{171}$ Fakultät für Physik und Astronomie, Julius-Maximilians-Universität, Würzburg, Germany

${ }^{172}$ Fachbereich C Physik, Bergische Universität Wuppertal, Wuppertal, Germany

${ }^{173}$ Department of Physics, Yale University, New Haven, CT, United States of America

${ }^{174}$ Yerevan Physics Institute, Yerevan, Armenia

${ }^{175}$ Domaine scientifique de la Doua, Centre de Calcul CNRS/IN2P3, Villeurbanne Cedex, France

${ }^{176}$ Faculty of Science, Hiroshima University, Hiroshima, Japan

${ }^{a}$ Also at Laboratorio de Instrumentacao e Fisica Experimental de Particulas-LIP, Lisboa, Portugal 
${ }^{\mathrm{b}}$ Also at Faculdade de Ciencias and CFNUL, Universidade de Lisboa, Lisboa, Portugal

${ }^{\mathrm{c}}$ Also at Particle Physics Department, Rutherford Appleton Laboratory, Didcot, United Kingdom

${ }^{\mathrm{d}}$ Also at TRIUMF, Vancouver BC, Canada

${ }^{\mathrm{e}}$ Also at Department of Physics, California State University, Fresno, CA, United States of America

${ }^{\mathrm{f}}$ Also at Novosibirsk State University, Novosibirsk, Russia

${ }^{\mathrm{g}}$ Also at Fermilab, Batavia IL, United States of America

${ }^{\mathrm{h}}$ Also at Department of Physics, University of Coimbra, Coimbra, Portugal

${ }^{\mathrm{i}}$ Also at Università di Napoli Parthenope, Napoli, Italy

${ }^{\mathrm{j}}$ Also at Institute of Particle Physics (IPP), Canada

${ }^{\mathrm{k}}$ Also at Department of Physics, Middle East Technical University, Ankara, Turkey

${ }^{1}$ Also at Louisiana Tech University, Ruston, LA, United States of America

${ }^{m}$ Also at Department of Physics and Astronomy, University College London, London, United Kingdom

${ }^{\mathrm{n}}$ Also at Group of Particle Physics, University of Montreal, Montreal QC, Canada

${ }^{\circ}$ Also at Department of Physics, University of Cape Town, Cape Town, South Africa

${ }^{\mathrm{p}}$ Also at Institute of Physics, Azerbaijan Academy of Sciences, Baku, Azerbaijan

${ }^{\mathrm{q}}$ Also at Institut für Experimentalphysik, Universität Hamburg, Hamburg, Germany

${ }^{\mathrm{r}}$ Also at Manhattan College, New York, NY, United States of America

${ }^{\mathrm{s}}$ Also at School of Physics, Shandong University, Shandong, China

${ }^{t}$ Also at CPPM, Aix-Marseille Université and CNRS/IN2P3, Marseille, France

${ }^{\mathrm{u}}$ Also at School of Physics and Engineering, Sun Yat-sen University, Guanzhou, China

${ }^{v}$ Also at Academia Sinica Grid Computing, Institute of Physics, Academia Sinica, Taipei, Taiwan

${ }^{\text {w}}$ Also at DSM/IRFU (Institut de Recherches sur les Lois Fondamentales de l'Univers), CEA Saclay (Commissariat a l'Energie Atomique), Gif-sur-Yvette, France

${ }^{\mathrm{x}}$ Also at Section de Physique, Université de Genève, Geneva, Switzerland

${ }^{y}$ Also at Departamento de Fisica, Universidade de Minho, Braga, Portugal

${ }^{\mathrm{z}}$ Also at Department of Physics and Astronomy, University of South Carolina, Columbia, SC, United States of America

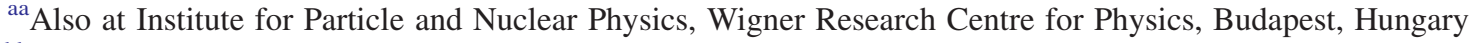

${ }^{\mathrm{bb}}$ Also at California Institute of Technology, Pasadena, CA, United States of America

${ }^{\mathrm{cc}}$ Also at Institute of Physics, Jagiellonian University, Krakow, Poland

${ }^{\mathrm{dd}}$ Also at LAL, Univ. Paris-Sud and CNRS/IN2P3, Orsay, France

${ }^{e e}$ Also at Department of Physics and Astronomy, University of Sheffield, Sheffield, United Kingdom

${ }^{\mathrm{ff}}$ Also at Department of Physics, Oxford University, Oxford, United Kingdom

${ }^{\mathrm{gg}}$ Also at Institute of Physics, Academia Sinica, Taipei, Taiwan

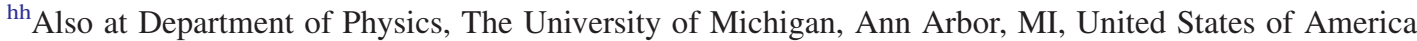

${ }^{\text {ii } A l s o ~ a t ~ L a b o r a t o i r e ~ d e ~ P h y s i q u e ~ N u c l e ́ a i r e ~ e t ~ d e ~ H a u t e s ~ E n e r g i e s, ~ U P M C ~ a n d ~ U n i v e r s i t e ́ ~ P a r i s-D i d e r o t ~ a n d ~ C N R S / I N 2 P 3, ~ P a r i s, ~}$ France

${ }^{\mathrm{jj}}$ Deceased. 